\title{
Non-Linear Contact Analysis of Meshing Gears
}

\author{
A Thesis \\ Presented to \\ The Faculty of California Polytechnic State University, \\ San Luis Obispo
}

\author{
In Partial Fulfillment \\ of the Requirements for the Degree \\ Master of Science in Mechanical Engineering
}

By:

Chun Hung Lee

June 2009 
(C) 2009

Chun Hung Lee

ALL RIGHTS RESERVED 


\section{COMMITTEE MEMBERSHIP}

TITLE:

Non-Linear Contact Analysis of Meshing Gears

AUTHOR:

Chun Hung Lee

DATE SUMMITED:

January 2010

COMMITTEE CHAIR: $\quad \mathrm{Xi} \mathrm{Wu}, \mathrm{Ph} . \mathrm{D}$.

Assistant Professor

Department of Mechanical Engineering

Cal Poly, San Luis Obispo

COMMITTEE MEMBER: Jim Meagher, Ph.D.

Professor

Department of Mechanical Engineering

Cal Poly, San Luis Obispo

COMMITTEE MEMBER: Peter Schuster, Ph.D.

Associate Professor

Department of Mechanical Engineering

Cal Poly, San Luis Obispo 


\section{Abstract}

\section{Non-Linear Contact Analysis of Meshing Gears}

\section{Chun Hung Lee}

Gear transmission systems are considered one of the critical aspects of vibration analysis, and it contains various potential faults such as misalignment, cracks, and noise. Therefore, it requires vibration monitoring to ensure the system is operating properly. Case mounted accelerometers are frequently used to monitor frequencies in a system. However, it is not a simple task to identify and interpret the acceleration data since there are many gear mesh frequencies present. One of the approaches utilized by researchers to perform gear diagnostic is Finite Element Modeling. This study focuses on stiffness cycle and meshing stiffness of non-linear quasi-static finite element modeling. The comparisons of meshing stiffness will concentrate on the type of elements, the integration methods, the meshing quality, plane stress and plane strain analysis, sensitivity of model tolerance, and crack modeling. The results show that the FEA approach is extremely sensitive to tolerance, mesh density, and element choice. Also, the results indicate that a complete sensitivity and convergence studies should be carried out for a satisfactory stiffness match. 


\section{Acknowledgments}

First, I would like to thank my adviser, Dr. Xi Wu, whose encouragement, guidance and genuine support from the initial to the final level enabled me to accomplish this project. Special recognition goes to Dr. Jim Meagher, Dr. Peter Schuster, and many other professors from the Mechanical Engineering Department who have assisted me at each stage of the project's development. I also would like to show my gratitude to Dr. Dewen Kong of Jilin University, for providing me technical support.

My parents, Chi Shing Lee and Po Fun Ma, deserve special mention for their inseparable support, caring, and gentle love. Gordon, thanks for being a supportive and caring sibling. Last but not least, I thank my beloved grandmother, Oi Lam, who is my foremost inspiration for pursuing this higher education degree. 


\section{Table of Contents}

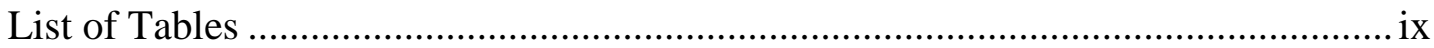

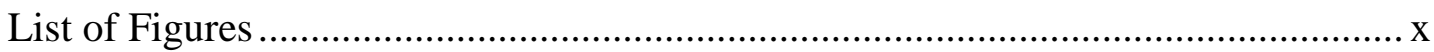

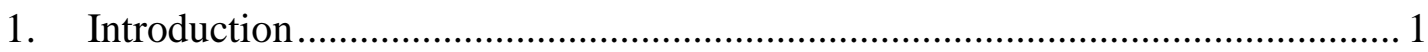

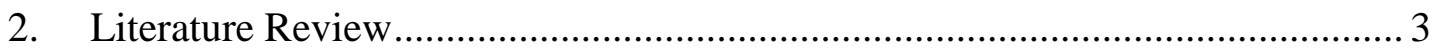

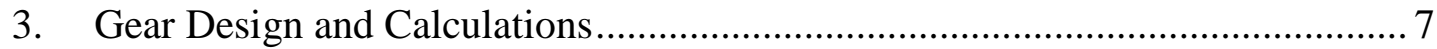

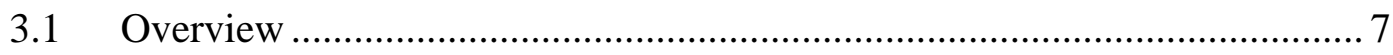

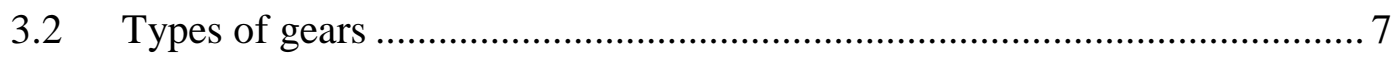

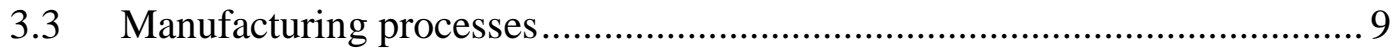

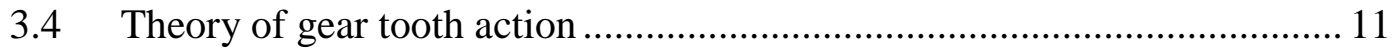

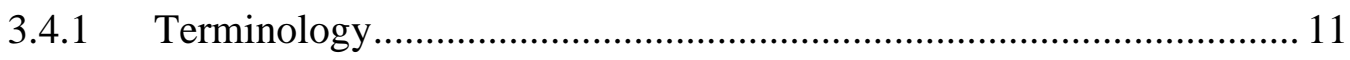

3.4.2 Line of action ........................................................................ 12

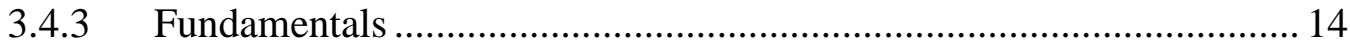

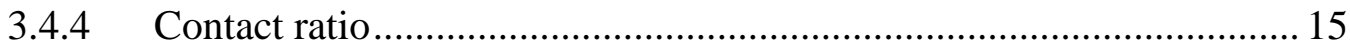

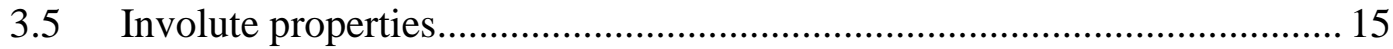

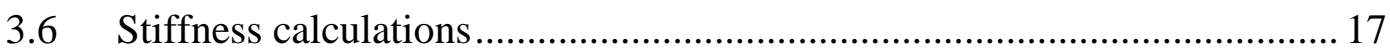

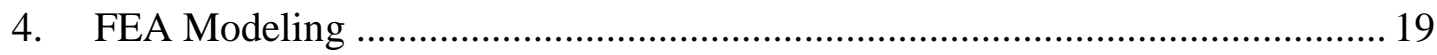

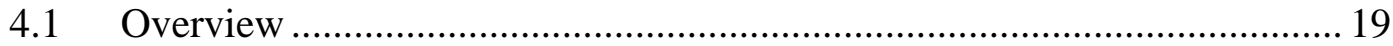

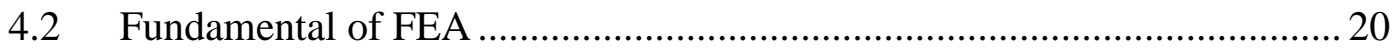

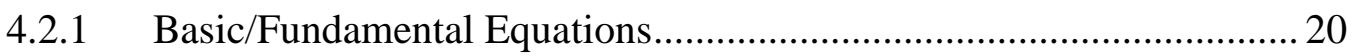

4.2.2 Procedures of FEA ..................................................................... 22

4.2.3 Various types of finite element methods ....................................... 25

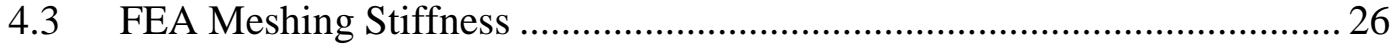

4.3.1 Torsional Mesh Stiffness ................................................................. 27

4.3.2 Linear Tooth Mesh Stiffness.......................................................... 31

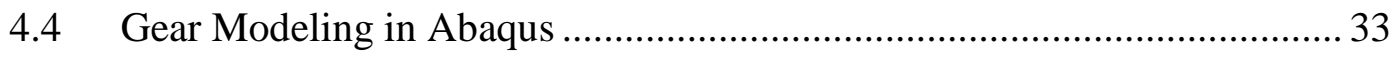




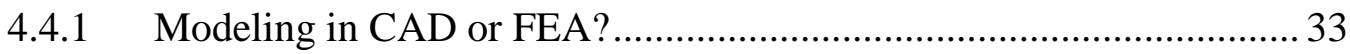

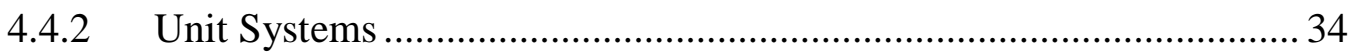

4.4.3 Input Parameters, Sketching, Assembling, and Meshing …………..... 35

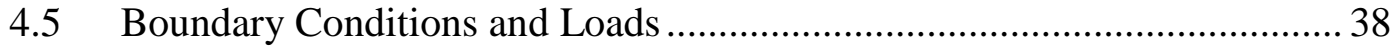

4.6 Defining Contact in Abaqus.................................................................... 43

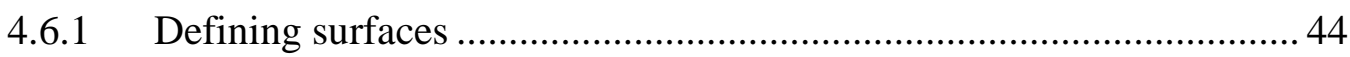

4.6.2 Contact interactions .......................................................................... 44

4.6.3 Small and Finite Sliding................................................................... 46

4.7 FEA Results and Comparisons.................................................................. 46

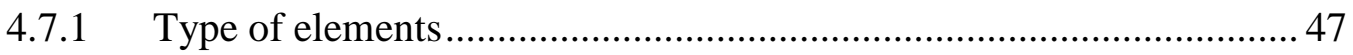

4.7.2 Integration methods ........................................................................ 51

4.7.3 Meshing quality ............................................................................... 55

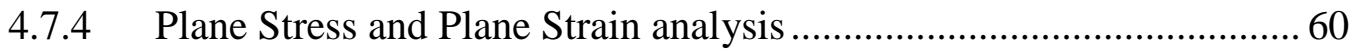

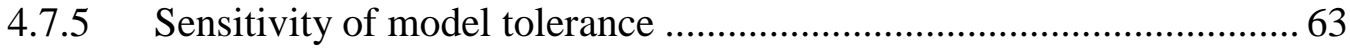

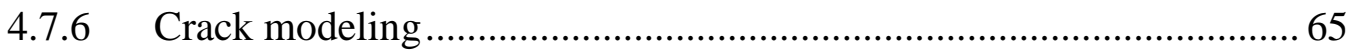

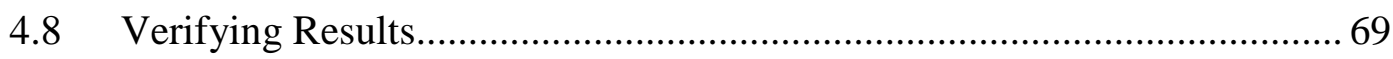

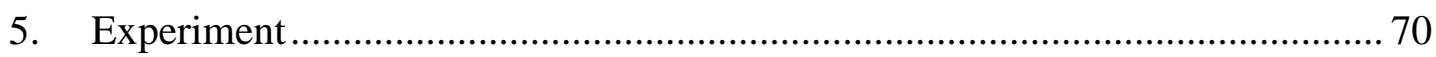

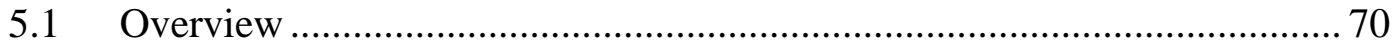

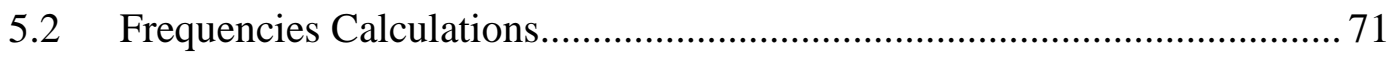

5.2.1 Gear Frequencies Calculations ………………….............................. 71

5.2.2 Bearing Frequencies Calculations....................................................... 72

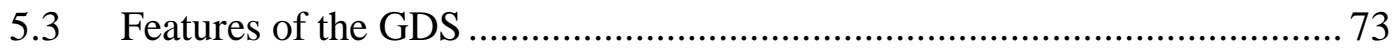

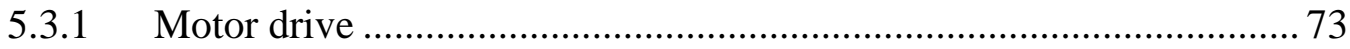

5.3.2 Speed control interface box ............................................................. 73

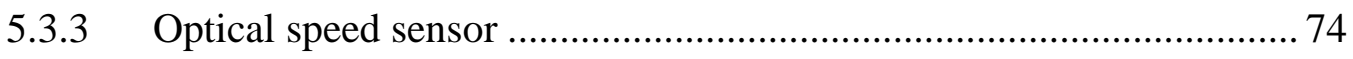

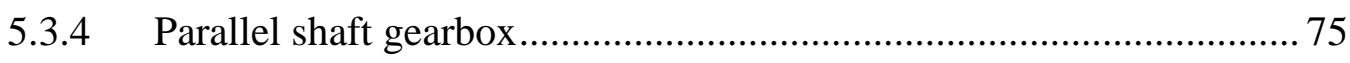

5.3.5 Braking mechanism ……………………………………………..... 75

5.4 Experimental Setup ............................................................................ 76

5.4.1 Gearbox Dynamics Simulator ........................................................... 76 
5.4.2 Bently Nevada Equipment ....................................................... 78

5.4.3 Accelerometers and ME'scopeVES............................................... 79

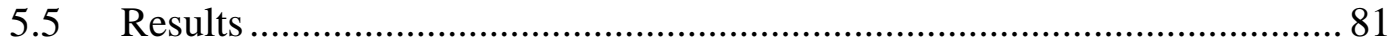

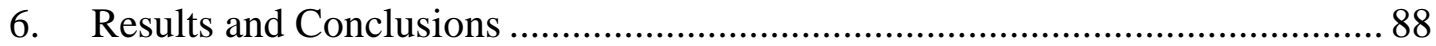

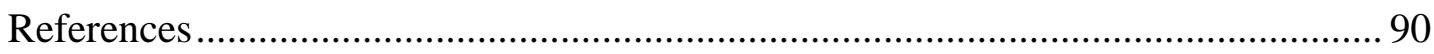

Appendix: MatLab Code for gear tooth profile .................................................. 92 


\section{List of Tables}

Table 1: Input Parameters for Low and High Contact Ratio Gears .......................... 16

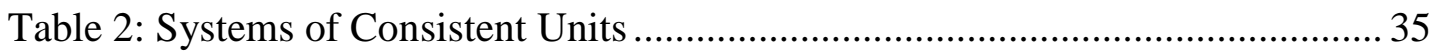

Table 3: Material Properties of Carbon Steel .......................................................... 35

Table 4: Input Parameters for Low and High Contact Ratio Gears ........................... 36

Table 5: Calculated Values vs. Experimental Values at $17 \mathrm{~Hz}$ Input Frequencies.... 81

Table 6: Calculated Values vs. Experimental Values at $25 \mathrm{~Hz}$ Input Frequencies.... 85 


\section{List of Figures}

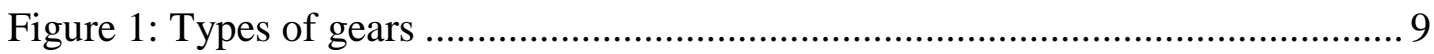

Figure 2: Nomenclature of spur gear teeth ........................................................... 11

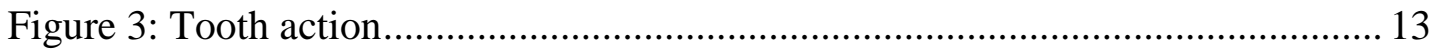

Figure 4: Gear tooth profile from MatLab ............................................................ 16

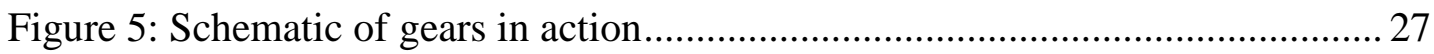

Figure 6: Coupling between the torsional and transverse motions of the gears ......... 32

Figure 7: Tooth and Associated Circles........................................................................ 36

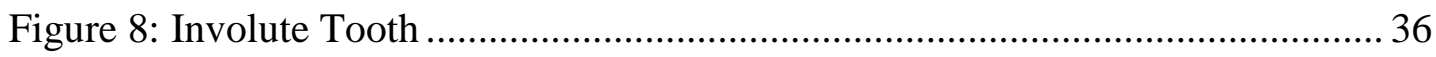

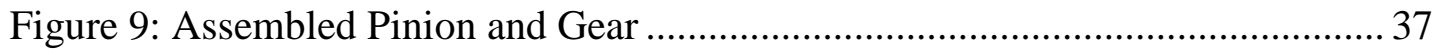

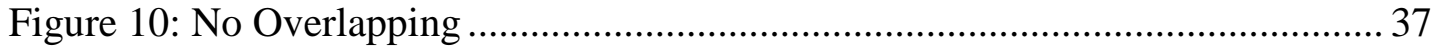

Figure 11: A Meshed Gear with Quadratic-Quad elements........................................ 38

Figure 12: Boundary Conditions and Applied Loads in dynamic analysis ................ 39

Figure 13: Boundary Conditions and Applied Load in quasi-static analysis.............. 41

Figure 14: Coupling constraint in Abaqus .................................................................. 43

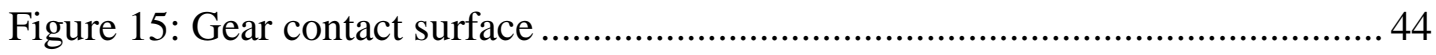

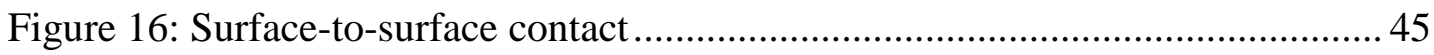

Figure 17: Commonly used element families .......................................................... 48

Figure 18: Node ordering and face numbering on elements ......................................4 49

Figure 19: Solid elements examples ..................................................................... 49

Figure 20: Linear tooth mesh stiffness responses in Linear and Quadratic elements 50

Figure 21: Integration points in element ............................................................. 52

Figure 22: Reduced Integration vs. Full Integration ...................................................52

Figure 23: Comparison of gear models with Reduced and Fully Integration.............54 
Figure 24: A model with biased seeding ...................................................... 56

Figure 25: A model meshed with quadrilateral elements and triangular elements.... 56

Figure 26: Coarse Mesh vs. Refined Mesh ..........................................................5

Figure 27: Linear tooth mesh stiffness changes with meshing quality .................... 60

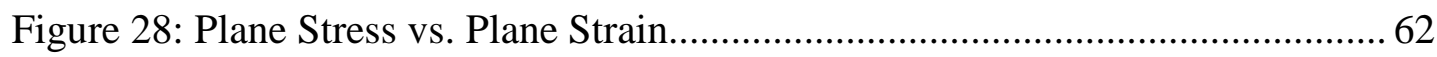

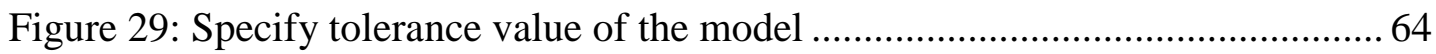

Figure 30: Mesh stiffness response to different tolerance values ...........................65

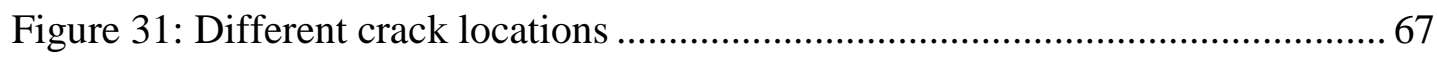

Figure 32: Mesh stiffness result with various crack scenarios .............................. 68

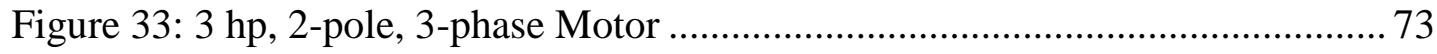

Figure 34: Delta VFD-B Speed Controller …................................................... 74

Figure 35: Banner Optical Speed Sensor ....................................................... 74

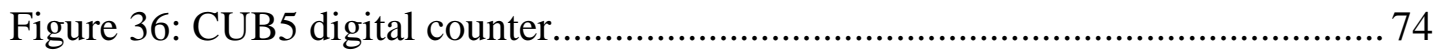

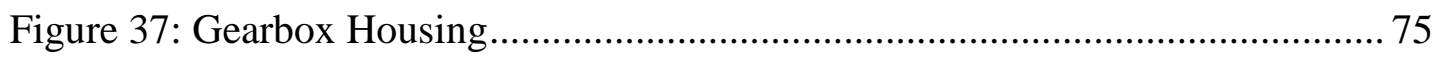

Figure 38: Parallel Shaft with Spur Gears ....................................................... 75

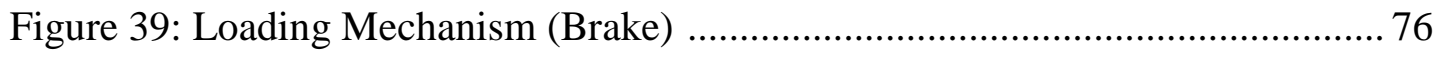

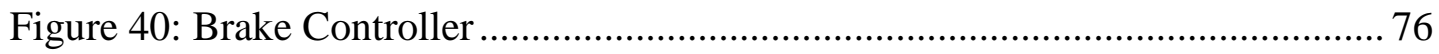

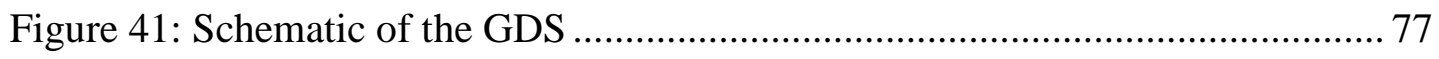

Figure 42: GDS Setup with ME'scopeVES and spectrum analyzer....................... 77

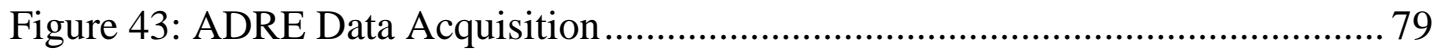

Figure 44: Proximity Probes Bracket............................................................... 79

Figure 45: Gearbox Assembly with Bracket and Hub ........................................ 79

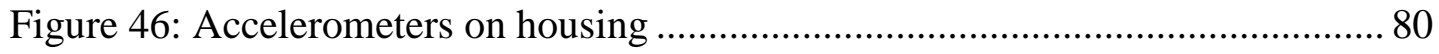

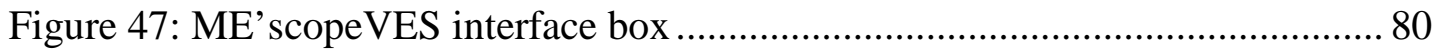

Figure 48: Power Spectrum Plot with frequency range between 0 to $2000 \mathrm{~Hz}$......... 82 
Figure 49: Power Spectrum Plot with frequency range between 1 to $700 \mathrm{~Hz}$.......... 83

Figure 50: Power Spectrum Plot with frequency range between 700 to $950 \mathrm{~Hz}$....... 83

Figure 51: Power Spectrum Plot with frequency range between 950 to $1500 \mathrm{~Hz} \ldots . .84$

Figure 52: Power Spectrum Plot with frequency range between 0 to $2000 \mathrm{~Hz}$........ 85

Figure 53: Power Spectrum Plot with frequency range between 0 to $750 \mathrm{~Hz}$........... 86

Figure 54: Power Spectrum Plot with frequency range between 700 to $1500 \mathrm{~Hz} \ldots . .86$

Figure 55: Power Spectrum Plot with frequency range between 1500 to $2000 \mathrm{~Hz} . . .87$

Figure 56: Comparison between FEA and Theoretical Models............................. 88 


\section{Introduction}

Gears are one of the oldest of humanity's inventions. Nearly all the devices we think of as a machines utilize gearing of one type or another. Gear technology has been developed and expanded throughout the centuries. In many cases, gear design is considered as a specialty. Nevertheless, the design or specification of a gear is only part of the overall system design picture. From industry's standpoint, gear transmission systems are considered one of the critical aspects of vibration analysis. The understanding of the behavior when gears are in mesh is extremely important if one wants to perform system monitoring and control of the gear transmission system. Although there are large amount of research studies about various topics of gear transmission, the basic understanding of gears in mesh still needs to be confirmed.

When a pair of gears mesh, localized Hertzian contact stress are produced along with tooth bending and shearing. This is a non-linear problem, and it can be solved by applying different types of contact elements and algorithms in finite element codes. However, due to the complicated contact conditions, acquiring results in the meshing cycle can be challenging since some solutions may not converge. In any case, using quadrilateral elements seem to be useful in solving gear contact problems with finite element analysis. Furthermore, meshing stiffness is often being discussed when a pair of gears are in mesh. Meshing stiffness can be separated into Torsional Mesh Stiffness and Linear Tooth Mesh Stiffness.

The torsional mesh stiffness is defined as the ratio between the input torque load and the angular displacement of the input gear. Once in mesh, the gears' pitch circles roll 
on each other without slipping. With a constant torque load, the torsional mesh stiffness changes through the rotation of the gears. These changes are due to the contact ratio between the pinion and gear. Depending on the contact ratio; the contact region would change and alternate from single tooth contact to double tooth contact or even a higher number of contacting pairs. This change of contact regions is referred to as a mesh cycle. Through the mesh cycle, the torsional mesh stiffness can be utilized as a tool to investigate gear transmission errors. Furthermore, the torsional mesh stiffness is related to the linear tooth mesh stiffness by the normal contact force that acts along the line of action. Basically, the linear tooth mesh stiffness of the gears is an easy approach to understand the coupling between the torsional and transverse motions of the system. The linear tooth mesh stiffness has been chosen as the primary parameter to be studied in this work.

This work is mainly focusing on, but not limited to, the gear modeling and analysis using the finite element method. Large amounts of FEA calculations were made using the finite element code - Abaqus. Comparisons between predicted linear tooth mesh stiffness are presented with different type of elements, integration methods, meshing quality, plane stress vs. plane strain, sensitivity of model tolerance, and crack modeling. In addition, small amount of experiments are performed in the aim of validating gearbox diagnostic methodologies. The objective of the experiments is to monitor and identify vibration frequencies associated with the gears and bearings in a gearbox. 


\section{Literature Review}

Gears are a critical component in the rotating machinery industry. Various research methods, such as theoretical, numerical, and experimental, have been done throughout the years regarding gears. One of the reasons why theoretical and numerical methods are preferred is because experimental testing can be particularly expensive. Thus, numerous mathematical models of gears have been developed for different purposes. This chapter presents a brief review of papers recently published in the areas of gear design, transmission errors, vibration analysis, etc., also including brief information about the models, approximations, and assumptions made.

Wyluda and Wolf [1] performed an elastic-plastic finite element analysis of the quasi-static loading of two acetal copolymer gears in contact. The applied load vs. gear set rotation is compared to actual experimental results. The geometry of the gear is modeled with variable thickness between the rim and web. Plane strain elements were used in the finite element model. Gear tooth failure is considered and modeled using methods of deactivating and separating elements when the tensile strength is exceeded. As a result, the mechanical behavior and prediction of copolymer acetal gears is quite complicated. Combination of computer simulations and component testing has merged a better understanding of copolymer acetal gear design. Also, the results indicate that a linear elastic approach is only suitable when the gears are under low loads and deformations. So, performing non-linear analysis is essential in order to optimize a gear set. 
In 2003, Barone et al. [2] aimed at investigating the behavior of a face gear transmission considering contact path under load, and load sharing and stresses, for an unmodified gear set including shaft misalignment and modification on pinion profile. The investigation is carried out by integrating a 3D CAD system and a FEA code, and by simulating the meshing of pinion and gear sectors with three teeth, using contact elements and an automated contact algorithm. The results show the influence of load on theoretically calculated contact paths, contact areas, contact length and load sharing. Also, it shows that the effectiveness of the numerical approach to the meshing problem in its complexity and that commonly adopted approaches are not suitable for non conventional, highly loaded gears in which rim and tooth deformations are not negligible. Overloads due to pinion misalignments and shift of contact areas are also being considered.

In 2001, Howard et al. [3] used a simplified gear dynamic model to explore the effect of friction on the resultant gear case vibration. The model includes the effect of variations in gear tooth torsional mesh stiffness, developed using finite element analysis, as the gears mesh together. The frictional force between teeth is integrated into the dynamic equations. Single tooth crack effects are shown on the frequency spectrum. The effect of the tooth crack could be seen in the time waveforms of all the dynamic variables being simulated when friction was neglected. The diagnostic techniques worked clearly when friction was included in the model, and in most cases friction gave a negligible change in the resulting values. 
In 2005, Wang and Howard [4] presented the methods and results of the use of FEA high contact ratio gears in mesh. The numerical models were developed with gears in mesh under quasi-static conditions. The details of transmission error, combined torsional mesh stiffness, load-sharing ratio, contact stress and tooth root stress against various input loads over a complete mesh cycle are also taking into account. Thus, various tooth profile modifications are presented and comparisons between the results show evidence for the optimal profile modification expected to gain the maximum benefit of high contact ratio gears. Also, the optimal relief length is normally dependent on the gears' geometrical properties. The results of optimal relief length vs. the tooth addendum variations have shown that the relief length can be very small, and it suggests that the contact ratio or the module be increased in order to retain the natural benefits of high contact ratio gears.

One year later (2006), Wang and Howard [5] investigated a large number of 2D and 3D gear models using finite element analysis. The models included contact analysis between teeth in mesh, a gear body, and teeth with and without a crack at the tooth root. The model results were compared using parameters such as the torsional mesh stiffness, tooth stresses and the stress intensity factors that are obtained under assumptions of plane stress, plane strain, and 3D analysis. Also, the models considered variations of face width of the gear. As a result, the finite element solution has been shown to produce acceptable results for stresses within a limited range. The $2 \mathrm{D}$ modeling errors can be significant when the gear is subject to a 
fracture such as a tooth root fatigue crack. Thus, 2D solutions may only apply in a very narrow range. Also, ignoring these errors (fatigue analysis) can lead to significantly erroneous results. The actual parameters used in the investigations demonstrate that caution must be taken where $2 \mathrm{D}$ assumptions are applied in the modeling.

In 2007, Carmignani et al. [6] have simulated the dynamic behavior of a faulted gear transmission. The meshing stiffness was evaluated statically as a function of the gear angular position using finite element gear meshing models. The deformation of the teeth under load and the faulted gears such as tooth cracks of different lengths at different locations on the tooth flank were taking into account in the simulations. Also, the numerical simulations were carried out in a simulink environment with different applied torques and gear angular velocities. As a result, the fracture causes a variation in the meshing stiffness when the faulty tooth is engaged in meshing. The crack affects stiffness only if the cracked zone is loaded between the tooth root and the contact point. However, if there are more teeth in contact, the uncracked teeth would share the load, which unloads the cracked tooth and thus reduces the stiffness disturbance effect. 


\section{Gear Design and Calculations}

\subsection{Overview}

The main purpose of gearing is to transmit motion from one shaft to another. If there is any mistake or error on the gears, motion will not be transmitted correctly. Also, if the errors on the gears are crucial, it may destroy or heavily damage the components in a gearbox. Therefore, it becomes important to understand the subject of gearing. In order to gain better understanding of gearing, one should get some knowledge about the design of gear and the theory of gear tooth action.

\subsection{Types of gears}

There are many different types of gears used by industry, but all these gears share the same purpose, which is to transmit motion from one shaft to another. Generally, gearing consists of a pair of gears with axes are either parallel or perpendicular. Among all the gears in the world, the four most commonly discussed gears are spur gear, helical gear, bevel gear, and worm gearing.

Spur gears considered as the simplest form of gearing, and they consist of teeth parallel to the axis of rotation. The common pressure angles used for spur gears are $14 \frac{1}{2}, 20$, and 25 degrees. One of the advantages of a low pressure angle is smoother and quieter tooth action. In contrast, larger pressure angles have the advantages of better load carrying capacity. 
Helical gears consist of teeth that are cut at an angle and inclined with the axis of rotation. Helical gears essentially have the same applications as spur gears. However, because of their gradual engagement of the teeth during meshing, helical gears tend to be less noisy. In addition, the inclined tooth develops thrust loads and bending couples, which are not present in the spur gear.

Bevel gears teeth are formed on conical surfaces, and unlike spur and helical gears, bevel gears are used for transmitting motion between intersecting shafts not parallel shafts. There are different types of bevel gears, but all of them establish thrust, radial, and tangential loads on their support bearings.

Worm gearing consists of the worm and worm gear. Depend upon the rotation direction of the worm; the direction of rotation of the worm gear would be different. The direction of rotation also depends upon whether the worm teeth are cut left-hand or right-hand. In general, worm gear sets are more efficient when the speed ratios of the two shafts are high. Basically, in worm gearing, higher speed equals to better efficiency. The following figure demonstrates the four most common types of gears in industry. 


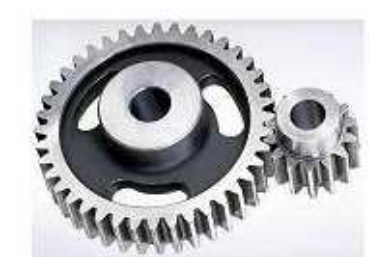

a. Spur Gears

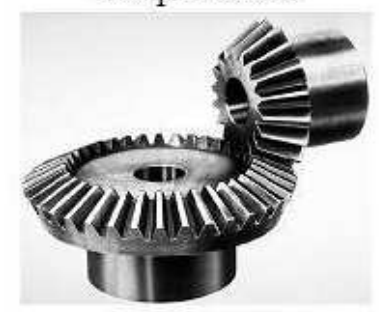

c. Bevel Gears

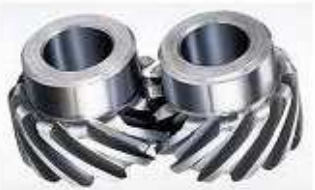

b. Helical Gears

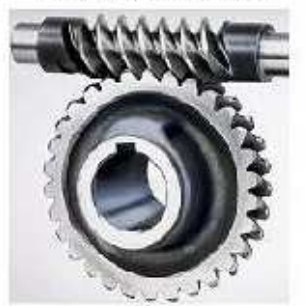

d. Worm Gears

Figure 1: Types of gears

(Images provided from www.howstuffworks.com)

\subsection{Manufacturing processes}

A number of ways can be used to manufacture the shape of the gear teeth; however, they can be classified into two categories - Forming and Generating. In forming processes, the tooth space takes the exact form of the cutter. On the other hand, generating is a process that uses a tool having a shape different from the tooth profile which is moved relative to the gear blank as to obtain the proper tooth shape. According to Drago [7], the same theoretical tooth forms can be produced by both forming and generating, but the actual profiles that result on the parts differ slightly. Generated profiles are actually a series of flats whose envelope is the desired form, while the surface of a formed profile is usually a continuous curve. In general, gear teeth may be machined by milling, shaping, or hobbing. Also, they may be finished by shaving, burnishing, grinding, or lapping. 
Milling - a form milling cutter will be used to conform the tooth space. The tooth form is produced by passing the milling cutter with the appropriate shape through the blank. The only drawback for this method is the necessity to use a different cutter for each gear because different gears have different-shaped tooth spaces.

Shaping - either a pinion cutter or a rack cutter will be used to generate the gear teeth. The cutter reciprocates with respect to the work and is fed into the gear bank. Since each tooth of the cutter is a cutting tool, the teeth are all cut after the blank has completed one rotation.

Hobbing - one of the fastest ways of cutting gears. The hob basically is a cutting tool that is shaped like a worm. As the hob rotates and feeds along the gear axis, the gear rotates about its axis in a carefully controlled environment. A single hob of a given normal pitch and pressure angle can be used to produce any standard external spur or helical gear with the same pitch and pressure angle.

Finishing - if there are errors in the tooth profiles, gears may be subjected to additional dynamic forces. A good finishing on tooth profiles would help to diminish these errors. Shaving machines offer to cut off a small amount of metal and improve the accuracy of the tooth profile. Burnishing utilizes hardened gears with slightly oversized teeth and run in mesh with the gear until the surfaces become smooth. Grinding employs the principle of generating and produces very accurate gear teeth. 
Lapping is applied to heat treated gears to correct small errors, improve surface finish, and remove nicks and burrs.

\subsection{Theory of gear tooth action}

\subsubsection{Terminology}

The first step of learning gear design is to know the basic terminology of the gear. Since spur gears are the most common form of gearing, it will be used to illustrate the nomenclature of gear teeth. The following figure is presented by Shigley et al. [8] and displays the nomenclature of spur gear teeth.

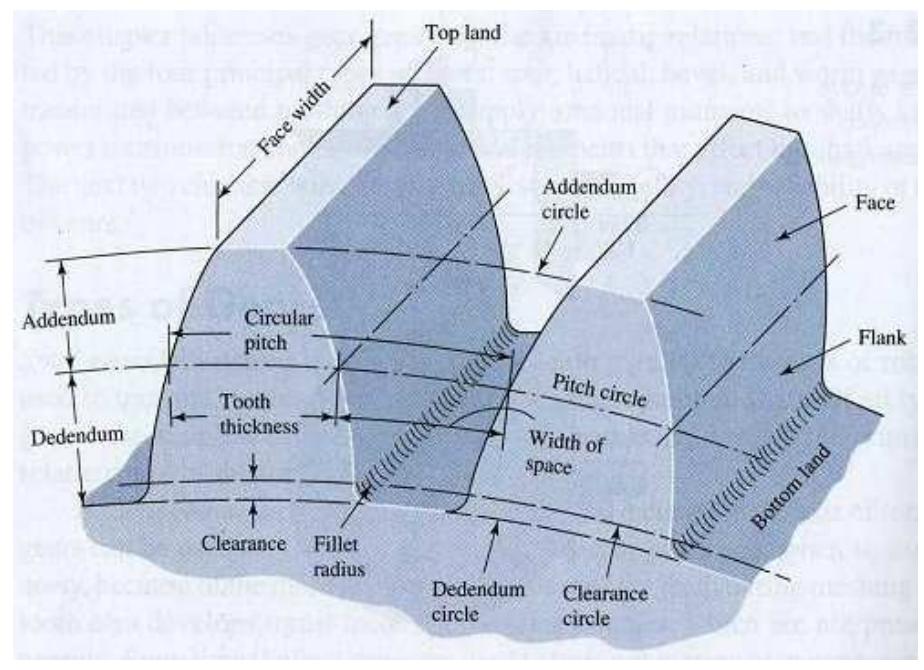

Figure 2: Nomenclature of spur gear teeth

(Shigley et al. [8], Figure 13-5, P.666)

One of the most important parameters on the gear teeth is the pitch circle since all calculations are based on this theoretical circle. The diameter of the pitch circle is called the pitch diameter $d$. When a pair of gears is mated together, the pitch circles of the gears are tangent to each other. The circular pitch $p$ is the distance on the 
circumference of the pitch circle between the corresponding points of adjacent teeth. Therefore, the circular pitch is the sum of the tooth thickness and the width of a space. The addendum is the radial distance between the pitch circle and the top of the tooth (top land). The dedendum is the radial distance between the pitch circle and the bottom of the tooth space (bottom land). The clearance is the amount by which the dedendum in a given gear exceeds the addendum of its mating gear. The diametral pitch $P$ is the ratio of the number of gear teeth to each inch of the pitch diameter. The module $m$ is the ratio of the pitch diameter to the number of teeth, and the unit of module is usually millimeter. Hence,

$$
\begin{aligned}
& P=\frac{N}{d} \\
& m=\frac{d}{N} \\
& p=\frac{\pi d}{N}=\pi m
\end{aligned}
$$

where,

$$
\begin{aligned}
& N=\text { Number of teeth } \\
& p=\text { Circular pitch } \\
& P=\text { Diametral pitch, teeth per inch } \\
& d=\text { Pitch diameter, inch } \\
& m=\text { Module }, \text { mm } \\
& d=\text { Pitch diameter }, \mathrm{mm}
\end{aligned}
$$

\subsubsection{Line of action}

When gear teeth are meshing against each other, it will generate rotary motion. Also, when a curved surface pushes against another, the point of contact appears where the 
two surfaces are tangent to each other. Imagine a line pierces through this contact point with the characteristic of being common normal to the surfaces. Then, the forces at any instant are directed along this line, and this line represents the direction of the forces. This is called the line of action or pressure line. Furthermore, the line of action will intersect the line of centers which is formed by the gears' centers at point $P$. This point is referred as the pitch point. The pitch point can be found by drawing the pitch circles of the gears since they are supposed to come in contact as soon as the gears are meshed together. The following figure shows the line of action and the tooth action of a pair of gears.

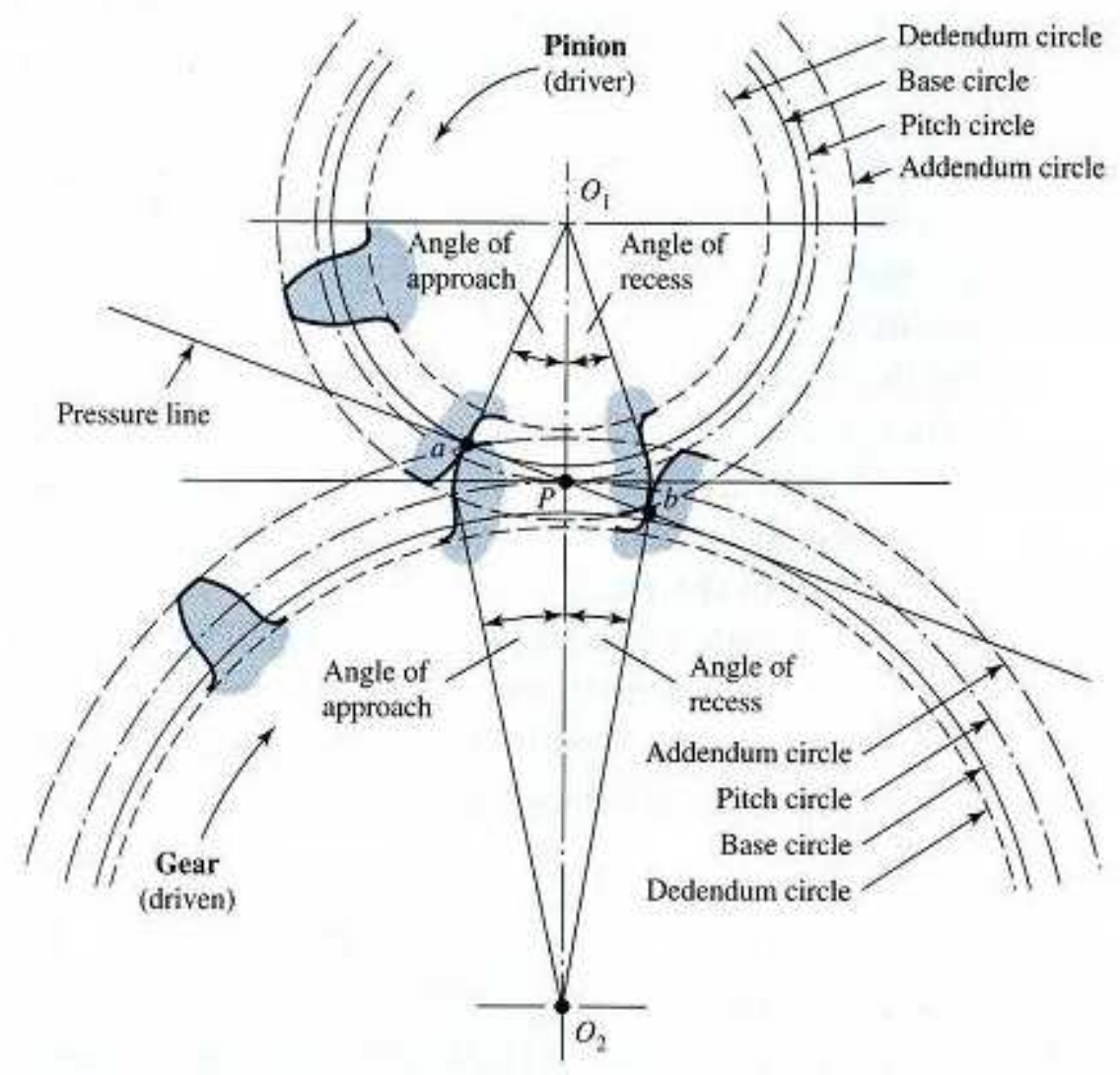

Figure 3: Tooth action

(Shigley et al. [8], Figure 13-12, P.671) 


\subsubsection{Fundamentals}

When two gears are meshed with each other, their pitch circles roll on one another without slipping. Thus the pitch line velocity can be defined as:

$$
V=r_{p} \omega_{p}=r_{g} \omega_{g}
$$

where $r_{p}$ and $r_{g}$ are the pitch radii of the pinion and gear; $\omega_{p}$ and $\omega_{g}$ are the angular velocities of the pinion and gear respectively. As shown in Figure 3.4.2, the pressure line is tangent to the base circles of the pinion and gear, and it pierces through the contact point. The horizontal line which is tangent to the pitch circles of the pinion and gear also pierces through the contact point. The angle between this horizontal line and the pressure line is identified as the pressure angle $\phi$. The pressure angle usually has values of $14 \frac{1}{2}, 20$, or 25 degrees. Furthermore, since the base circles are tangent to the pressure line, using basic geometry, the base circle radius can be determined through the pitch radius and the pressure angle.

$$
r_{b}=r \cdot \cos \phi
$$

For standard gear teeth, the addendum and dedendum distances are $1 / P$ and $1.25 / P$ respectively. The clearance, as previous described, is equal to the dedendum distance minus the addendum distance. In order to draw a tooth, one must know the tooth thickness. The tooth thickness is measured on the pitch circle and can be calculated as:

$$
t=\frac{p}{2}
$$




\subsubsection{Contact ratio}

The contact between the gears begins and ends at the intersections of the two addendum circles with the line of action. Depending on the design of the gears and the contact ratio, sometimes there will be two or more teeth in contact. According to Machinery's Handbook [9], the contact ratio is the ratio of the arc of action in the plane of rotation to the circular pitch. Often, it is considered as a number that indicates the average number of pairs of teeth in contact. Furthermore, the contact ratio is obtained most directly as the ratio of the length of action to the base pitch.

$$
m_{c}=\frac{L_{a b}}{p_{b}}=\frac{L_{a b}}{p \cdot \cos \phi}
$$

\subsection{Involute properties}

The involute curve of a tooth plays an important role in gear design and analysis. For instance, friction and wear between two gears is dependent on the profile of the teeth; the uniform velocity ratio is also dependent on the tooth profile. The involute tooth allows the center distance or spacing of the gears to vary over some range without affecting the velocity ratio. Therefore, an accurate gear tooth profile will lead to high quality results. Even though the formation of the involute tooth profile has been described in [7] and [8], it is still challenging to construct the correct gear tooth profile in CAD and FEA code environment. However, with the up-to-dated numerical programs, one is able to develop a reliable code to create the gear tooth profile [10]. With some adjustments [11], the code can accurately generate the profile of a gear tooth. The details of the code can be found in the Appendix at the end of this work. The code only requires users to provide few parameters of the gear: 
diametral pitch, pressure angle, and number of teeth. The code will generate a series of points represent the $\mathrm{x}$ - and $\mathrm{y}$ - coordinates of the tooth involutes with the origin located at the center of the gear. Figure 3.5.1 shows an example of a gear tooth profile generated from MatLab. These series of points will then be imported into CAD and FEA code environment in order to create a gear model for vibration analysis. In addition, two sets of gears are considered for analysis. One with low contact ratio while the other with high contact ratio. The following table shows the input parameters of the two sets of gears.

Table 1: Input Parameters for Low and High Contact Ratio Gears

\begin{tabular}{|c|c|c|c|c|}
\cline { 2 - 5 } \multicolumn{1}{c|}{} & \multicolumn{2}{c|}{ Low Contact Ratio } & \multicolumn{2}{c|}{ High Contact Ratio } \\
\cline { 2 - 5 } \multicolumn{1}{c|}{} & Pinion & Gear & Pinion & Gear \\
\hline Diametral Pitch, P (teeth/inch) & 10 & 10 & 12 & 12 \\
\hline Pressure Angle (degree) & 20 & 20 & 14.5 & 14.5 \\
\hline Number of teeth & 23 & 31 & 24 & 60 \\
\hline
\end{tabular}

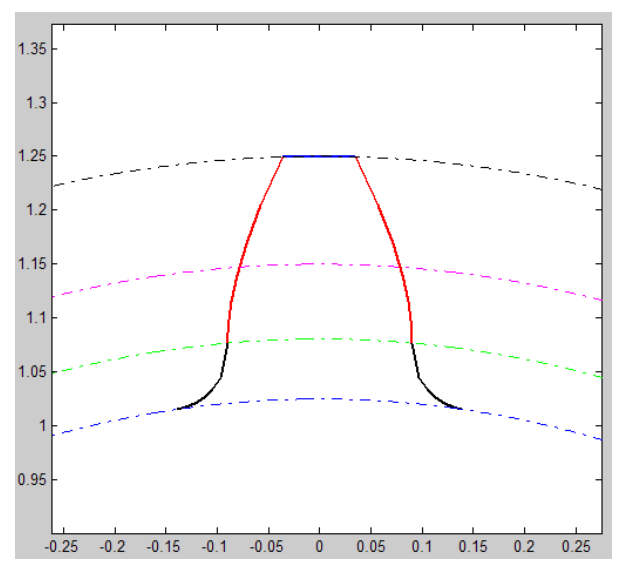

Figure 4: Gear tooth profile from MatLab 


\subsection{Stiffness calculations}

The ultimate goal in this work is to numerically predict the mesh stiffness of a pair of gears. In another words, finite element gear models will be created and analyzed in order to investigate the non-linearity of the meshing gears. However, as a general rule, FEA results should always be compared to closed-form solution or experimental testing in term of result validating. The closed-form solution used to calculate the meshing stiffness is basically the application of Strain Energy and Castigliano's Theorem. When the gears are in mesh, the contact force acts along the line of action. The contact force can be decomposed into tangential and radial forces. To sum up, the gear tooth is treated as a short cantilever beam with intermediate load. Since it is a short beam, the shear bending term becomes more significant, thus, it must be taken into account in the calculation. Keep in mind that the actual gear tooth shape is not included in the calculation, it is simply a short rectangular beam. This calculation is just a rough estimate of the meshing stiffness and in used as partial validation of FEA results.

By using Strain Energy method and Castigliano's Theorem, the stiffnesses of the pinion tooth and gear tooth can be obtained. The gear set can be modeled as springs connected in series. Then, the meshing stiffness can be calculated by the following equation,

$$
K_{m}=\frac{K_{p} \cdot K_{g}}{K_{p}+K_{g}}
$$

where $K_{p}$ and $K_{g}$ are the pinion tooth stiffness and the gear tooth stiffness respectively. The detail calculations can be found in the Appendices section, and the 
result of this closed-form solution is used to compare with the FEA results in the conclusion section. 


\section{4. $\quad$ FEA Modeling}

In engineering, Finite Element Analysis (FEA) is a numerical method to interpolate an approximate solution to a boundary value problem. FEA of meshing gears is subjected to non-linear contact analysis. The contact between the gear and the pinion is highly non-linear because the surfaces of the tooth could come in and out of contact in a sudden manner. Also, depending on the boundary conditions, loads, frictional responses, and other factors, it will make the solution convergence extremely difficult since all of these areas could introduce non-linearity to the problem.

\subsection{Overview}

In this chapter, a large amount of FEA calculations were made using Abaqus. The main objective is to calculate the Linear Tooth Mesh Stiffness for a gear-pinion pair in mesh. The comparisons of Linear Tooth Mesh Stiffness will concentrate on the type of elements, the integration methods, the meshing quality, plane stress and plane strain analysis, sensitivity of model tolerance, and crack modeling. Two different gear-pinion models were considered; one with low contact ratio and one with high contact ratio. Furthermore, the stresses near the contact areas and the root of tooth are three dimensional when a pair of gears in mesh. However, many FEA gear models have reduced the problem to two dimensions because of the computational time, efficiency, and cost. Therefore, two dimensional assumptions were applied to all the FEA models in this section, and all the models were considered with thickness of 1 inch because of simplicity. 


\subsection{Fundamental of FEA}

\subsubsection{Basic/Fundamental Equations}

In the theory of elasticity, there are fundamental concepts, definitions, and equations used in the analysis of stress and deformation. Both classical and finite element methods will be used to solve problems with these fundamentals. In order to judge the shortcomings or range of applicability of approximate solutions, elasticity theory states the following categories that must be met by an exact solution. The five categories are the basic or fundamental equations that are needed for a boundary value problem.

1. Equilibrium

2. Compatibility

3. $\quad$ Stress - Strain

4. $\quad$ Strain - Displacement

5. Boundary Conditions

Equilibrium is defined by a set of equations whereby the forces on the differential elements balance under applied forces. These forces mainly come from the stresses on the edges and/or from the body forces. However, in many problems, the effects of loads applied to the surface of the structure are far more important than the effects of the body forces.

Compatibility is a condition met automatically if the displacements and $1^{\text {st }} \& 2^{\text {nd }}$ derivatives are continuous. Compatibility condition can be defined as the 
displacement field being continuous and single valued. Physically, it means there is no breaking, no cracks in stretching, no kinks in bending, and no part overlapping when a body is deformed. Furthermore, most finite element methods are based on displacements rather than stresses, which mean each element conforms to a displacement field that is continuous and single valued.

Stress - Strain (Constitutive law). When ignoring the effect of temperature change, the stress-strain relations can be defined as Generalized Hooke's Law: stress is directly proportional to strain.

$$
\begin{aligned}
& \{\sigma\}=[E]\{\varepsilon\} \\
& \{\varepsilon\}=[C]\{\sigma\}
\end{aligned}
$$

where $[E]$ is a symmetric matrix of material stiffnesses (stiffness matrix), $[C]$ is a symmetric matrix of material compliances (compliance matrix), and $[E]=[C]^{-1}$. However, this rule is an approximation limited to small strains and certain materials. For an isotropic material, it has no preferred directions, and the material properties are commonly expressed as a combination of two of the following: modulus of elasticity $E$, Poisson's ratio $v$, and modulus of rigidity (shear modulus) G. For an orthotropic material, an anisotropic material that displays the distinct values of stiffness in the perpendicular directions, these directions are referred to the principal directions of the material. For instance, imagine a chunk of wood is being cut from a log. The axial direction of the wood will be the stiffest, the radial direction will be intermediate stiff, and the circumferential direction will be the least stiff. 
Strain - Displacement. Strains may be classified into two categories: normal strain and shear strain. A normal strain is caused by a normal stress which acts perpendicular to the surface of a material. By definition, normal strain is the ratio of change in length to original length. Therefore,

$$
\begin{gathered}
\text { Strain }=\text { Change in length } / \text { Original length } \\
\qquad \varepsilon=\frac{\Delta L}{L}
\end{gathered}
$$

In contrast, shear strain is a strain that acts parallel to the surface of a material, and is defined as the amount of change in a right angle. Regardless whether it is normal strain or shear strain, the relationship between strain and displacement is an important factor in the formulation of finite elements for stress analysis problems.

Boundary Conditions prescribed the restriction of displacement and of stress at the boundary of the structure. Surface traction are a type of boundary condition where stress is defined on a boundary.

\subsubsection{Procedures of FEA}

Prior to the results of the FEA calculations, it is important to understand the general procedures of gear modeling in Abaqus. Just like other finite element analysis programs, Abaqus has the capability to analyze stress, displacement, and other parameters in a given system. Without being too specific, the following are the steps or procedures on how finite element programs perform calculations on a system:

1. Divide a complex system into small pieces (elements).

2. Formulate equations for each element (Equilibrium, Stress-Strain, etc.). 
3. Assemble the connected elements to form one big set of equations.

4. Apply external loads and boundary conditions.

5. Solve for nodal displacement of the system.

6. Calculate stresses, strains, reaction forces, etc. at nodes.

After breaking up a continuum structure into discrete pieces; these pieces are referred as elements where a deformation field is assumed within each element. Also, all elements share deformation at a common node which is used to fasten the elements together. Eventually, the displacement field will be written in terms of nodal displacement.

$$
\{u\}=[N]\{d\}
$$

where $\{u\}$ is the displacement field, $\{d\}$ is the nodal displacement, and $[N]$ is the shape functions. Shape Functions are used to serve as interpolation functions. In general (for common elements), the shape functions can be obtained from Lagrange Polynomials. From strain - displacement, strain is defined as change in length over original length. As the length gets small, the strain can be re-defined as $\varepsilon_{x}=\frac{d u}{d x}$ (1D, x-direction). Therefore, in 2D matrix form, the strains can be written as,

$$
\{\varepsilon\}=[\partial]\{u\}
$$

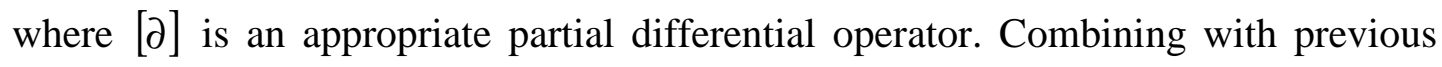
equation, it yields:

$$
\begin{gathered}
\{\varepsilon\}=[\partial][N]\{d\} \\
\{\varepsilon\}=[B]\{d\}
\end{gathered}
$$


where $[B]$ is the strain displacement matrix.

Ultimately, stiffness of the system is required to calculate the nodal displacement of the system. So, it is essential to find the system's stiffness matrix.

Consider the energy stored in a spring which is commonly known as,

$$
U=\frac{1}{2} k x^{2}
$$

with $k$ being the spring stiffness, and $x$ being the change in distance. The energy can be written in matrix equivalent form:

$$
U=\frac{1}{2}\{d\}^{T}\left[K_{e}\right]\{d\}
$$

where $\left[K_{e}\right]$ is the element stiffness matrix or local stiffness matrix, and $\{d\}$ again is the nodal displacement. Also, since energy is conserved, it must equal to the internal energy which is defined in matrix form as,

$$
U_{i}=\frac{1}{2} \int_{V}\{\varepsilon\}^{T}\{\sigma\} d V
$$

Hence,

$$
\begin{aligned}
& \frac{1}{2}\{d\}^{T}\left[K_{e}\right]\{d\}=\frac{1}{2} \int_{V}\{\varepsilon\}^{T}\{\sigma\} d V \\
& \{d\}^{T}\left[K_{e}\right]\{d\}=\int_{V}\{d\}^{T}[B]^{T}[E][B]\{d\} d V \\
& {\left[K_{e}\right]=\int_{V}[B]^{T}[E][B] d V}
\end{aligned}
$$

After each element stiffness matrix in the system is computed, the next step is to assemble all the connected elements to form one big set of equations - Stiffness Equations, which is a set of $\mathrm{n}$ algebraic equations with n-unknowns: 


$$
[K]\{D\}=\{F\}
$$

where $[K]$ and $\{D\}$ are the global stiffness matrix and the nodal displacement vector in the global level respectively. $\{F\}$ is the external force vector or applied nodal forces. The objective is to solve for nodal displacement $\{D\}$ of the system, but it cannot be solved until the equation has been modified to account for the boundary conditions. Unless there are enough nodal displacements that are fixed to prevent the system from moving as a rigid body under external loading, the set of nodal displacements will remain unsolvable. With that being said, after applying the external loads and boundary conditions to the system, the nodal displacement vector can be solved from:

$$
\{D\}=[K]^{-1}\{F\}
$$

\subsubsection{Various types of finite element methods}

In FEA, there are various methods that can be used to solve the problems. For instance, there are variable size method (h-method), polynomial degree method (pmethod), and the hybrid method (hp-method). So, the question arises as what are the differences between all these methods, and why would one choose a particular method over another?

The variable size method, or h-method, is the most common technique. This method engages splitting elements in space while keeping their polynomial degree fixed. In another words, it increases the number of elements in the chosen areas. In theory, as the number of elements increase (approach infinity) in the finite element model, the 
error or inaccuracy between the FEA solution and the exact solution is expected to become zero.

The polynomial degree method or p-method is a method that increases the polynomial degree of the finite element shape functions. The p-method has been shown to be more robust when compare to the h-method over problems that include non-linear applications. One of the significant differences is its ability to improve the results for any mesh automatically, which means there are no requirements for userdefined meshing control.

The hp-method or hp-adaptive solution basically combines adaptively elements with h-method and p-method in order to achieve quick exponential convergence rates. This exponential convergence makes the hp-method a very attractive choice because most other finite element methods only converge with an algebraic rate. In hpmethod, the element can be subdivided geometrically, and various polynomial degrees can be applied to the sub-elements. Or, just increase its polynomial degree without subdividing the element in space. This hp-method allows users to construct a model with h-method to obtain global responses while using p-method to improve the solution accuracy over the areas of particular interest.

\subsection{FEA Meshing Stiffness}

Throughout the years, many different procedures have been developed to model gears meshing behaviors. Most of the published finite element analysis models have analyzed a single tooth gear or partial gear, and identical gears have been applied to 
both pinion and gear. It is understandable to use only part of the gear and identical gears for finite element analysis due to complexity and computational time. However, it is not practical since there is no incomplete gear in industries, and identical gears would only produce one-to-one gear ratio. With the continuing software and hardware developments, the modeling and analyzing capabilities in Abaqus have improved significantly. Therefore, the whole body of the gears in mesh should be modeled. Also, different sizes of gear should be applied to pinion and gear in order to achieve desired gear ratio to simulate the gear set in reality.

\subsubsection{Torsional Mesh Stiffness}

When a pair of gears meshes, one of the important factors is the torsional mesh stiffness variation as the gears teeth rotate through the mesh cycle. Once in mesh, the gears' pitch circles roll on one another without slipping. The following figure demonstrates the motion of a pair of meshing gears:

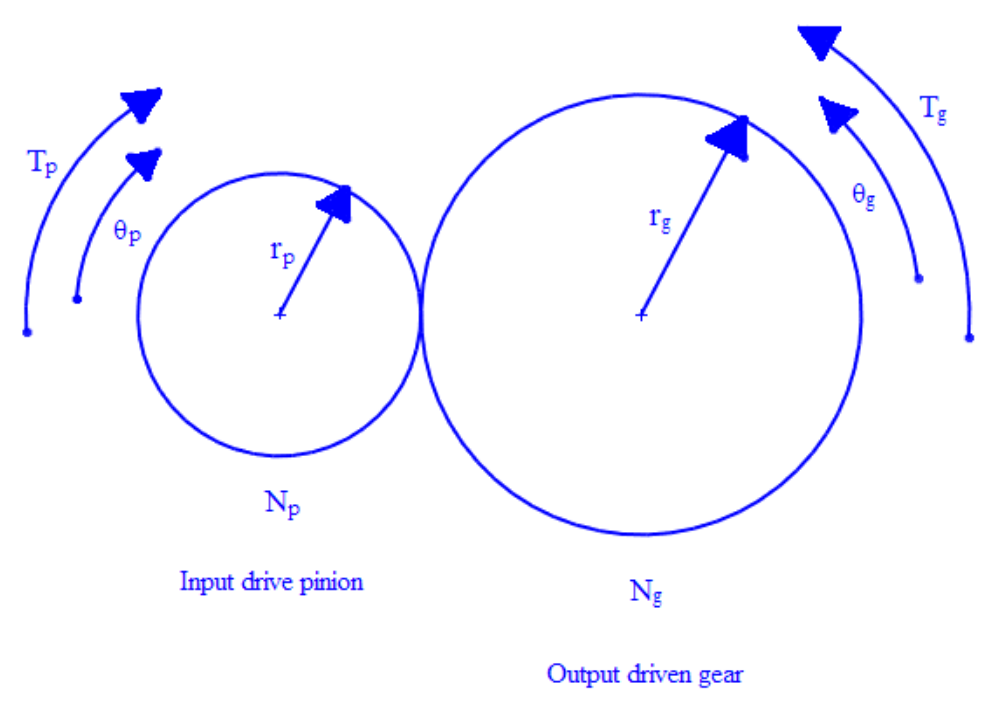

Figure 5: Schematic of gears in action 
where,

$$
\begin{aligned}
& T_{P}=\text { Input pinion torque, in-lbf } \\
& \theta_{P}=\text { Angular displacement of pinion, rad } \\
& r_{P}=\text { Pitch radius of pinion, in } \\
& N_{P}=\text { Number of teeth of pinion } \\
& T_{g}=\text { Output gear torque, in-lbf } \\
& \theta_{g}=\text { Angular displacement of gear, rad } \\
& r_{g}=\text { Pitch radius of gear, in } \\
& N_{g}=\text { Number of teeth of gear }
\end{aligned}
$$

As gears turn, the distance traveled along each gears' circumference is the same. Thus,

$$
r_{P} \theta_{P}=r_{g} \theta_{g}
$$

The ratio of the number of teeth along the circumference is in the same proportion as the ratio of the radii which yield the following relationship:

$$
\frac{N_{P}}{N_{g}}=\frac{r_{P}}{r_{g}}=\frac{\theta_{g}}{\theta_{P}}
$$

Furthermore, the rotational energy is defined as the Torque times angular displacement, and with the assumption that there is no energy dissipation:

$$
\begin{gathered}
T_{P} \theta_{P}=T_{g} \theta_{g} \\
\frac{T_{g}}{T_{P}}=\frac{\theta_{P}}{\theta_{g}}=\frac{N_{g}}{N_{P}}
\end{gathered}
$$


The definition of stiffness, $k$, is a measure of resistance of an elastic body to deformation produced by an applied force such as bending, shear, tension or compression, etc. Thus,

$$
k=\frac{F}{\delta}
$$

where, $\quad F$ is the steady force applied on the body, lb

$\delta$ is the displacement produced by the force, in

In general, the displacement can refer to a point distinct from where the force is applied. However, a complicated structure will not deflect purely in the same direction as an applied force. In that case, it would take extremely long time to solve complex problems by hand calculation. Because of that, it is better to use numerical methods to solve complicated structure problems. Furthermore, a body may also have a rotational stiffness, $k_{T}$, which primarily caused by torsion:

$$
k_{T}=\frac{T}{\theta}
$$

where, $\quad T$ is the applied torque on the body, lb-in

$\theta$ is the angular displacement, $\mathrm{rad}$

From the above equation, the torsional mesh stiffness can be seen as the ratio between the torque and the angular displacement.

For finite element analysis, there are different approaches to simulate the gears meshing cycle. Furthermore, there are two different analysis procedures within Abaqus; one is Abaqus/Standard analysis procedure while the other is Abaqus/Explicit analysis procedure. Abaqus/Standard procedure is mainly used to 
solve static problems with simple structures. In contrast, Abaqus/Explicit is a procedure that should be selected when solving dynamic complex structures problems. Thus, the ideal Abaqus procedure that should be selected for solving gear meshing problems is Abaqus/Explicit analysis procedure. It is because the pinion and gear are relatively complex structures, and they are in fact rotating. However, there is one significant draw back or concern in using Abaqus/Explicit analysis procedure, and it is the computational time. Assuming the gear model would converge without any major error, the convergence rate would become extremely slow become of its complex structures and motions. As one can imagine, when the gear teeth come in contact, there would be impacts on the teeth. These impacts or impact points are constantly changing as long as the gear set is rotating. Depending on the gear ratio; one gear meshing cycle could vary between 10 to 40 degrees (rough estimation). In this meshing cycle, there are tremendous among of calculations take place. And because of these calculations, the computational time in Abaqus/Explicit dynamic analysis is much longer than those in Abaqus/Standard static analysis.

In addition, another way to predict the torsional mesh stiffness of two gears in mesh is to use Quasi-Static method. Quasi-Static is a condition that refers to forces or displacements which vary or change slowly with time. A force is considered to vary slowly if the frequency of variation is much lower than the lowest natural frequency of the system. Despite that the gear rotates, it may be solved as static problem in order to reduce the computational time and the computer capability. Therefore, torque will be applied on the pinion, and its angular displacement will be measured 
so that the torsional mesh stiffness can be calculated using equation 4.21. One might ask if the gear model is being solved statically, how is the meshing cycle being simulated. The answer is by conducting a series of FEA calculations with different gear positions. Previous work by Howard and Wang [3] has stated that the torsional mesh stiffness of gears in mesh at particular positions throughout the mesh cycle was generated by rotating both solid gears, then creating a finite element model in that particular position. Then both gears will be rotated to another position; another finite element model will be created for torsional mesh stiffness, and so on. Ultimately, the torsional mesh stiffness will be plotted against the rotation of the pinion or gear in order to show the meshing cycle. In Abaqus, this Quasi-Static analysis will be performed using the Abaqus/Standard analysis procedure; the torsional mesh stiffness, as mentioned before, will be obtained from input a torque at the pinion hub, while the gear hub is restrained from rotating. The external applied forces and boundary conditions will be discussed later in the section.

\subsubsection{Linear Tooth Mesh Stiffness}

Based on previous research such as Howard and Wang [3] \& [4] and Carmignani et al. [6], the linear tooth mesh stiffness of the gears is an easy way to understand the coupling between the torsional and transverse motions of the system. The following figure shows the coupling between the torsional and transverse motions of the gears: 


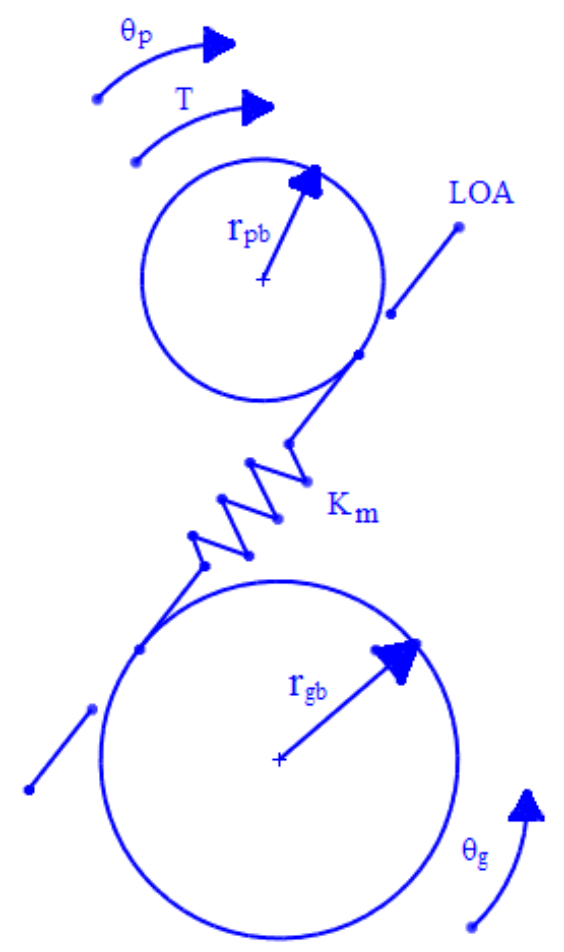

Figure 6: Coupling between the torsional and transverse motions of the gears where,

$$
\begin{aligned}
& T=\text { Input torque, in-lbf } \\
& r_{p b}=\text { Base circle radius of pinion, in } \\
& \theta_{P}=\text { Angular displacement of pinion, rad } \\
& r_{g b}=\text { Base circle radius of gear, in } \\
& \theta_{g}=\text { Angular displacement of gear, rad } \\
& K_{m}=\text { Linear tooth mesh stiffness, lb/in } \\
& L O A=\text { Line of action }
\end{aligned}
$$

To derive the linear tooth mesh stiffness, it needs to start from the torsional mesh stiffness. The torsional mesh stiffness is related to the linear tooth mesh stiffness by the normal contact force that acts along the line of action which connects the base circles of the pinion and gear. Once again, the torsional mesh stiffness can be defined as the ratio between the torque and the angular deflection. Now, consider there is a 
contact force $F$, which acts along the line of action. The torque $T$ will then equal to the contact force multiply by the perpendicular distance, in this case, base circle radius of pinion $r_{p b}$. Thus, $T=F r_{p b}$. Furthermore, with the small angle approximation, the angular displacement $\theta$ can be defined as $\theta=s / r_{p b}$, where $s$ is the arc length of the base circle. Therefore, the torsional mesh stiffness can then be rewritten as the following:

$$
K_{T}=\frac{T}{\theta}=\frac{F r_{p b}}{s / r_{p b}}=\frac{F r_{p b}{ }^{2}}{s}=K_{m} r_{p b}^{2}
$$

From equation 4.22, the linear tooth mesh stiffness $K_{m}$, can be seen as the ratio of the contact force $F$ to the linear displacement $s$ along the line of action. Notice that the linear displacement $s$ is equal to the arc length for small angle $\theta$. Therefore, the relationship between the linear tooth and torsional mesh stiffness becomes:

$$
K_{m}=\frac{K_{T}}{r_{p b}^{2}}
$$

The linear tooth mesh stiffness $K_{m}$, will be the primary parameter in this finite element study. Different cases and scenarios will be applied to the gear model, and the results will be compared and commented accordingly.

\subsection{Gear Modeling in Abaqus}

\subsubsection{Modeling in CAD or FEA?}

With the basic understanding of how finite element programs work, a finite element model must be created with appropriate parameters such as dimensions, loads, constraints, element choice, mesh selection, etc. In a way, creating the finite element model is the most time consuming step of finite element analysis. Users should 
spend time to create the model as accurately as possible since geometry is one of the critical aspects in FEA. In Abaqus, there are two different methods to construct the model. The first method is to build the model in a computer-aided design (CAD) environment such as SolidWorks, Pro/ENGINEER, or CATIA, and export the model with a file format such as IGES, ACIS, or Parasolid. The file is then imported into Abaqus for set up and analysis. However, the main disadvantage for this method is the CAD geometry data could be lost during the translation of the model, which means the dimensions of the model are no longer exact. The second method is to use Abaqus' internal drawing capabilities to build the model. In this method, no geometry data is lost since the file does not need to be translated. However, the modeling functions in Abaqus are not as good as the other CAD programs; users often encounter difficulties for building complex models due to the interface limitations. It would be ideal if the models are built in a CAD environment and no geometry data are lost during translation.

\subsubsection{Unit Systems}

For this research, the second method was chosen because a large amount of geometry data was lost during translate from CAD files. The gear tooth involutes are no longer exact after being imported into Abaqus. Therefore, gears will be drawn entirely in Abaqus. Before defining any model, it is important to decide which system of units will be used. Since Abaqus has no built-in system of units, all input data must be specified in a consistent fashion. The following table included some common systems of consistent units. 
Table 2: Systems of Consistent Units

\begin{tabular}{|c|c|c|c|c|}
\hline Quantity & SI & $\mathrm{SI}(\mathrm{mm})$ & US Unit (ft) & US Unit (inch) \\
\hline Length & $\mathrm{m}$ & $\mathrm{mm}$ & $\mathrm{ft}$ & in \\
\hline Force & $N$ & $N$ & lbf & lbf \\
\hline Mass & $\mathrm{kg}$ & tonne $\left(10^{3} \mathrm{~kg}\right)$ & slug & $\mathrm{lbf} \mathrm{s}^{2} / \mathrm{in}$ \\
\hline Time & $\mathrm{s}$ & $\mathrm{s}$ & $s$ & $\mathrm{~s}$ \\
\hline Stress & $\mathrm{Pa}\left(\mathrm{N} / \mathrm{m}^{2}\right)$ & $\mathrm{MPa}\left(\mathrm{N} / \mathrm{mm}^{2}\right)$ & $\operatorname{lbf} / f \mathrm{t}^{2}$ & psi $\left(\operatorname{lbf}^{\prime} / \mathrm{in}^{2}\right)$ \\
\hline Energy & $J$ & $\mathrm{~mJ}\left(10^{-3} \mathrm{~J}\right)$ & $\mathrm{ft}$ lbf & in lbf \\
\hline Density & $\mathrm{kg} / \mathrm{m}^{3}$ & tonne/mm ${ }^{3}$ & slug/ $/ \mathrm{ft}^{3}$ & lbf $s^{2} / \mathrm{in}^{4}$ \\
\hline
\end{tabular}

Users need to be careful with the unit of density when using the "US Unit". The densities from textbooks, material handbooks, or World Wide Web are often multiplied by the acceleration due to gravity. US Unit (inch) will be used to build the gear models in this section. The selected material for the gears is carbon steel since it is one of the most commercially use steel in the industries. The material properties of carbon steel are shown in Table 3.

Table 3: Material Properties of Carbon Steel

\begin{tabular}{|c|c|}
\hline Modulus of Elasticity (Mpsi) & 30 \\
\hline Modulus of Rigidity (Mpsi) & 11.5 \\
\hline Poisson's Ratio & 0.292 \\
\hline Unit Weight (lbf/in $\left.{ }^{3}\right)$ & 0.282 \\
\hline Mass Density $\left(\mathrm{lbf} \mathrm{s}^{2} / \mathrm{in}^{4}\right)$ & 0.00073 \\
\hline
\end{tabular}

\subsubsection{Input Parameters, Sketching, Assembling, and Meshing}

With the correct unit system, model the gears as accurately as possible. However, the model should be simplified by deleting features that have less significant affect on the results, e.g. keyways. The most difficult part is to draw the involutes of the gear 
tooth. Use the MatLab code from previous chapter and input the correct parameters. The input parameters for the gears with low and high contact ratio are shown in Table 4.

Table 4: Input Parameters for Low and High Contact Ratio Gears

\begin{tabular}{|c|c|c|c|c|}
\cline { 2 - 5 } \multicolumn{1}{c|}{} & \multicolumn{2}{c|}{ Low Contact Ratio } & \multicolumn{2}{c|}{ High Contact Ratio } \\
\cline { 2 - 5 } \multicolumn{1}{c|}{} & Pinion & Gear & Pinion & Gear \\
\hline Diametral Pitch, P (teeth/inch) & 10 & 10 & 12 & 12 \\
\hline Pressure Angle (degree) & 20 & 20 & 14.5 & 14.5 \\
\hline Number of teeth & 23 & 31 & 24 & 60 \\
\hline
\end{tabular}

The code will generate a single tooth and the circles of pitch, base, addendum and dedendum. Figure 7 and Figure 8 show the tooth and the circles that generated from the MatLab code.

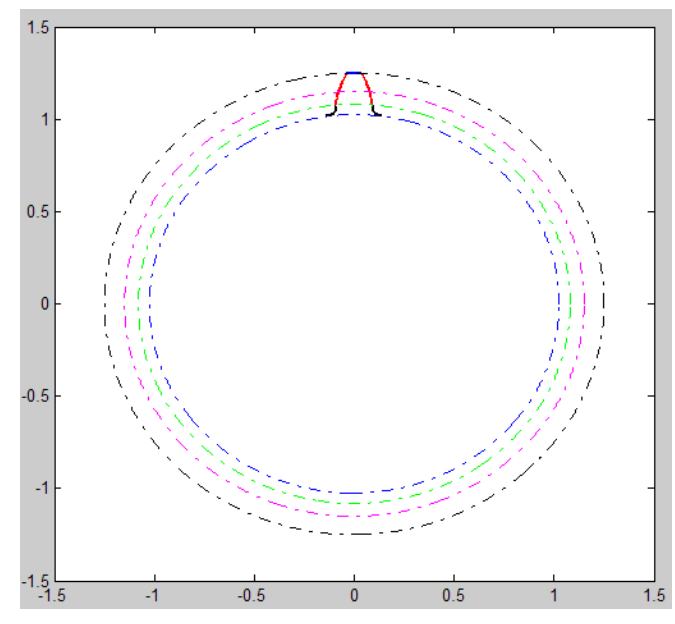

Figure 7: Tooth and Associated Circles

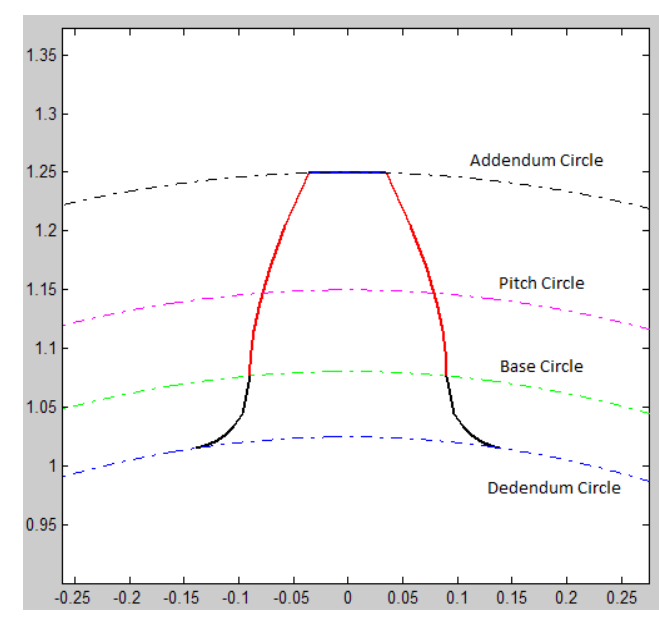

Figure 8: Involute Tooth

The series of points represent the $\mathrm{x}$ - and $\mathrm{y}$-coordinates of the tooth involutes with the origin located at the center of the gear. The $\mathrm{x}$ - and $\mathrm{y}$-coordinates will be placed into Abaqus and connected with the Spline function. The remaining parts should be relatively straightforward to sketch. The pinion and the gear will need to be 
assembled correctly in order to perform analysis. Usually, the actual position of the contact is unknown. However, the pitch circles of pinion and gear should be in contact once they are assembled. Use the Translate Instance function to adjust the distance so that the pitch circles are in contact. Also, the teeth of the pinion and gear should not overlap. Use the Rotate Instance function to adjust the gear teeth so that they are not overlapping the pinion teeth. The following figures have shown the assembled gear set and its proper positions.

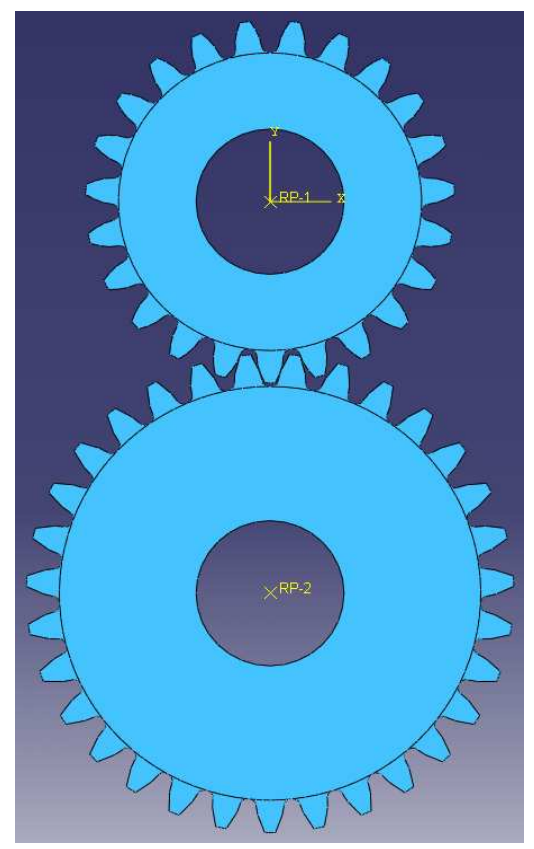

Figure 9: Assembled Pinion and Gear

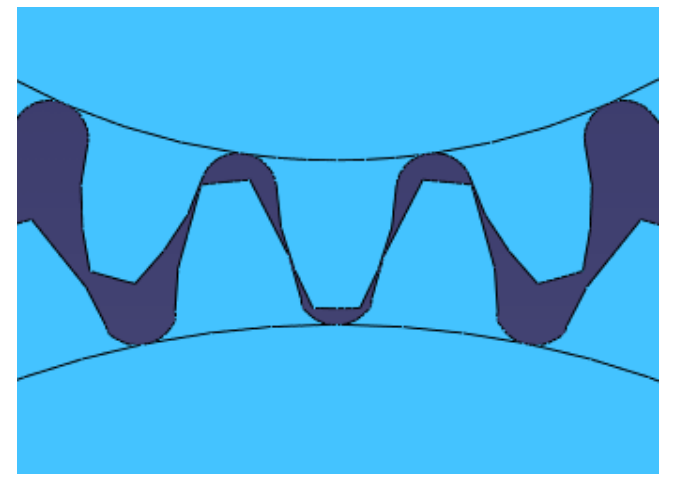

Figure 10: No Overlapping

Once the geometrical model is available, it is necessary to create meshes for analysis process. It is crucial to use adequate finite element mesh because it has big impact on the model convergence. Using proper element types and assigning quality meshes are the essential steps to build adequate mesh, and they will be discussed in details later on in the chapter. The following is an example of a meshed gear. 


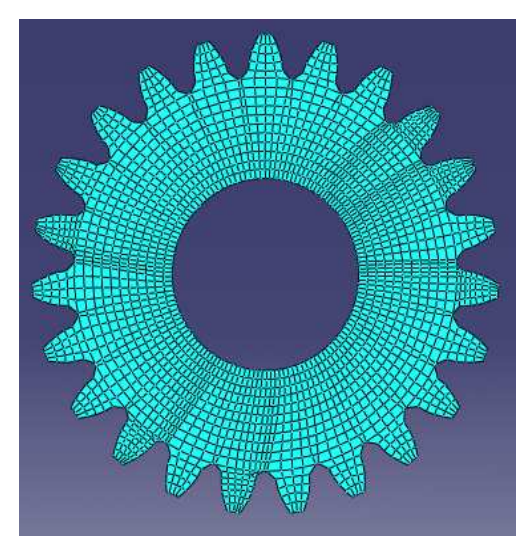

Figure 11: A Meshed Gear with Quadratic-Quad elements

\subsection{Boundary Conditions and Loads}

The next step in the process is to apply the boundary conditions and loads onto the system. The boundary conditions should be applied in a way that closely matches or simulates the gears' motions as the loads act on the system. As one might suspect, are there any different ways to apply the boundary conditions on the gear model? The answer is: Yes, there are! Depending on what procedures you choose in Abaqus, the boundary conditions are applied slightly differently. If Abaqus/Explicit procedure has been chosen, all degree of freedoms (DOF) should be restricted for both pinion and gear except the rotational degree of freedom. Therefore, both pinion and gear are free to rotate. The following schematic has shown the appropriate boundary conditions and applied loads on the gear model in Abaqus/Explicit dynamic analysis procedure. 


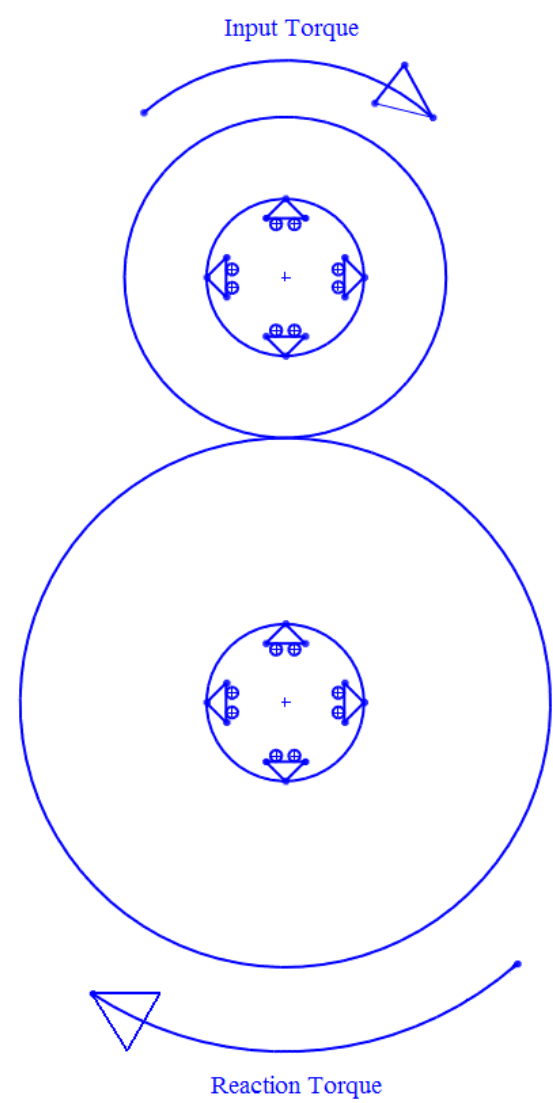

Figure 12: Boundary Conditions and Applied Loads in dynamic analysis

As shown in Figure 12, the input torque is applied on the pinion, while the reaction torque is applied on the gear. In general, the input torque $(T)$ is calculated through power $(P)$ and angular speed $(\Omega)$, and can be defined as such:

$$
\begin{gathered}
\text { Power }=\text { Torque } \times \text { Angular Speed } \\
P=T \cdot \Omega
\end{gathered}
$$

For different units of power, torque, or angular speed, a conversion factor must be inserted into the above equation. For instance,

$$
P=\frac{T \cdot \Omega}{5252}
$$


where, $\quad P$ has a unit of horsepower (hp)

$T$ has a unit of foot-pounds (ft-lbf)

$\Omega$ has a unit of revolutions per minute (rpm)

The conversion factor 5252 comes from $(33,000 \mathrm{ft}-\mathrm{lbf} / \mathrm{min}) /(2 \pi \mathrm{rad} / \mathrm{rev})$. In this particular research, power $=1.5 \mathrm{hp}$ and angular speed $=1800 \mathrm{rpm}$ have been selected. Hence, from equation 4.25 , the input torque can be calculated to $4.38 \mathrm{ft}-1 \mathrm{bf}$. However, for consistent unit purposes, the input torque unit should be converted to in-lbf. Therefore, the input torque should equal to 52.521 in-lbf (Note: this torque value will be used in all FEA gear models in this research). Furthermore, for low contact ratio gear model, the number of teeth of pinion and gear are 23 and 31 respectively. Using equation 4.19 , the output gear torque or the reaction torque can be calculated to 131.303 in-lbf.

For this research, Abaqus/Standard procedure has been chosen; Quasi-Static method should be applied to the gear model. The boundary conditions and loads should be applied as such: Pinion - restrict all DOFs except the rotational degree of freedom. Gear - completely restrict all DOF. The only load that applies on the model is the input torque, and it is applied on the pinion. The following figure demonstrates the boundary conditions and applied load on the gear model in Abaqus/Standard quasistatic analysis procedure. 


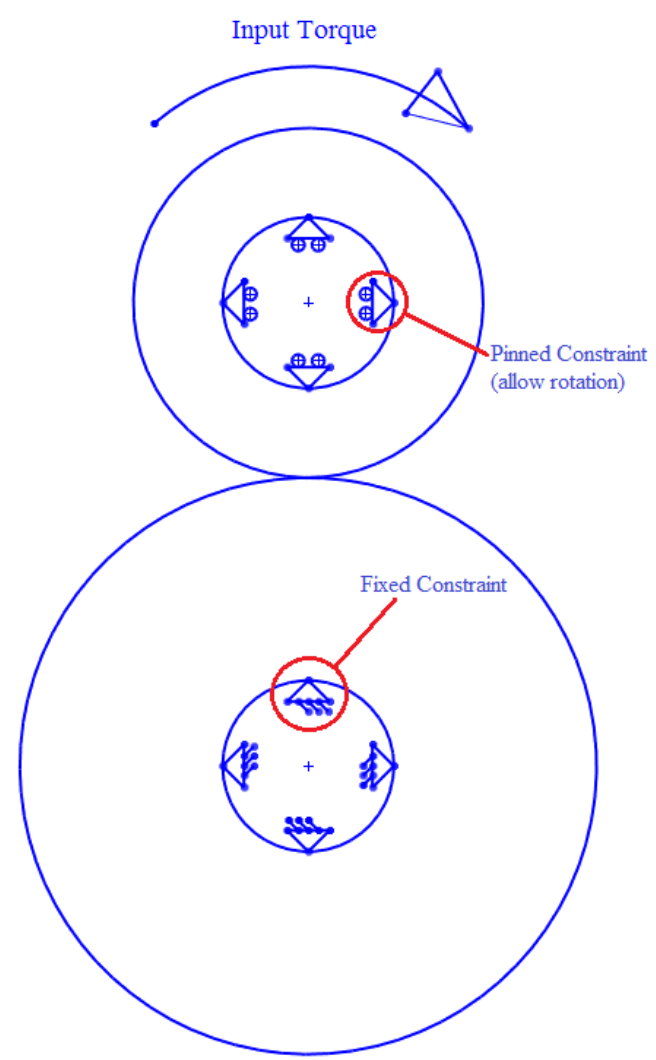

Figure 13: Boundary Conditions and Applied Load in quasi-static analysis

Since it is Quasi-Static analysis, a series of FEA calculations with different gear positions will be conducted in order to capture the stiffness changes through the meshing cycle. Therefore, after one FEA calculation, both pinion and gear will rotate to another position for the next calculation. Because the pinion and gear have different among of teeth, their rotation will be different. For simplicity purpose, the pinion will rotate at an equal increment fashion, and the gear will rotate accordingly based on the gear ratio. In another words, after one FEA calculation, the pinion will rotate $1^{\circ}$ clockwise, and the gear will rotate $0.7419^{\circ}$ (gear ratio is 23/31) counterclockwise for the next calculation. This step will be repeated until the meshing cycle is completed. The meshing cycles from previous work, Howard and 
Wang [3] \& [4], are between $20^{\circ}$ to $25^{\circ}$. However, their work is based on pairs of identical gear. This research has concentrated on non-identical, whole body, and full teeth gears. So, taking into account the non-identical gear issue, the pinion will be rotate from $0^{\circ}$ to $40^{\circ}$ in the FEA calculations so that the full meshing cycle is considered.

Since shafts are not explicitly modeled, the input torque and load are handled with constraints. In Abaqus, there is a function called "Coupling" which enable user to couple two objects together. While coupling, user can define which DOF(s) of the objects should be coupled together. In the gear model case, the center node of the gears should be coupled with the gears' hubs. In order to have the same motions, all DOFs of the gears' hubs must be coupled with the center node DOFs. Hence, the input torque can now be applied on the pinion center node; the boundary conditions can be applied on the gear center node and pinion center node respectively. The following figure shows the coupling process between the center node and the gear hub. 


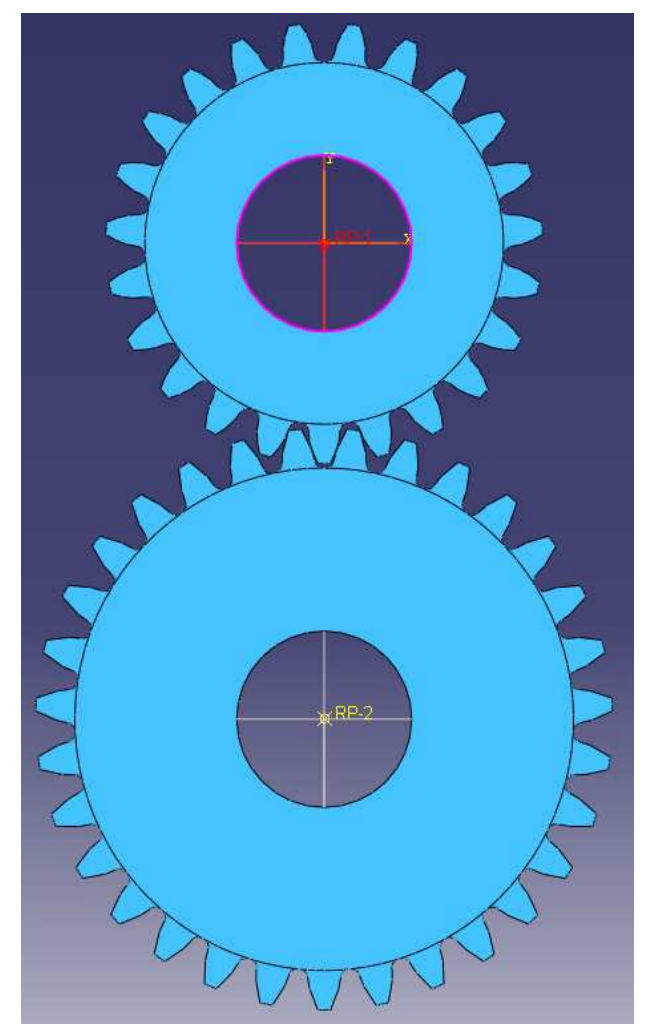

Figure 14: Coupling constraint in Abaqus

\subsection{Defining Contact in Abaqus}

In engineering, there are a lot of problems involving contact between two or more components. The main purpose of contact simulations is to identify the areas on the surfaces that are in contact and to calculate the contact pressures generated. In FEA, contact conditions can be described as a special class of discontinuous constraint. The constraint is discontinuous because it is applied only when the two surfaces are in contact. So, when the two surfaces separate, no constraint is applied. Therefore, it is important that Abaqus be able to detect if two faces are in contact and apply the contact constraints accordingly. According to Abaqus [12], different contact simulations are integrated into the interface. In Abaqus/Standard, contact simulations 
are either surface based or contact element based. In Abaqus/Explicit, it utilizes either the general contact algorithm or the contact pair algorithm.

\subsubsection{Defining surfaces}

Regardless which method is being used, surfaces that will be involved in contact must be created, and they are created from the element faces of the underlying material. For instance, in two or three dimensional solid continuum elements, contact surfaces are specified by selecting the regions of a part. Rigid surfaces are the surfaces of rigid bodies which can be defined as an analytical shape. For the gear model, the contact surfaces are the surfaces of the gear teeth. The following figure shows the contact surfaces defined in Abaqus as red.

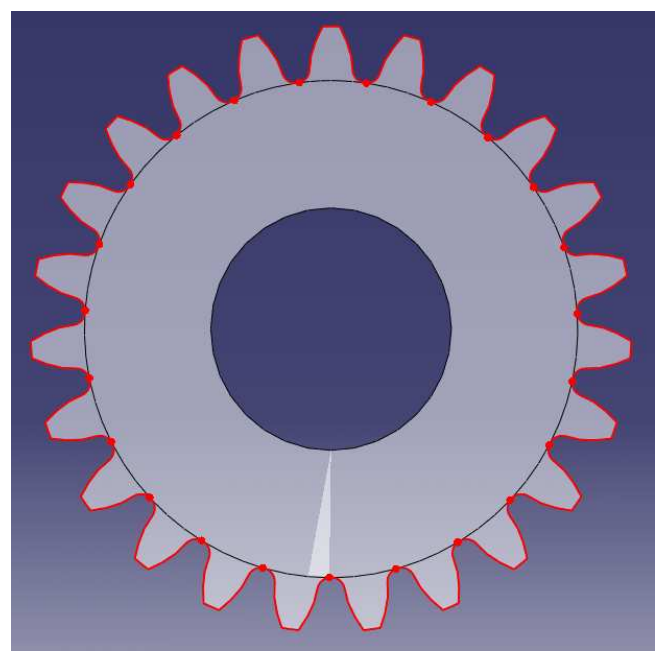

Figure 15: Gear contact surface

\subsubsection{Contact interactions}

After the contact surfaces are created, the next step is to create Surface-to-surface contact interaction which pairs the surfaces that come in contact with each other. The interaction between the contacting surfaces includes the normal and tangential components. Each contact interaction can refer to a contact property that specifies a 
model for its contacting surfaces. For the gear model, a "Hard Contact" option is applied as the normal behavior; a "Friction" is added to take into account of the sliding in the tangential behavior. Furthermore, Abaqus/Standard utilizes a masterslave contact algorithm. The algorithm states that only the master surface can penetrate the slave surface between slave nodes as the surfaces come in contact. So, in order to achieve the best possible contact simulation, users must be careful to select the master and slave surfaces. Simple rules to select the surfaces are: the slave surface should be the softer one, and the slave surface should be the more finely meshed surface. Since the pinion and the gear from the model are made out of the same material, and their mesh densities are similar, it is hard to judge which surface should be the master or slave. So, the pinion teeth surface is chosen to be the master surface without a specific reason. Figure 16 shows the contact interaction of a pair of gears in Abaqus.

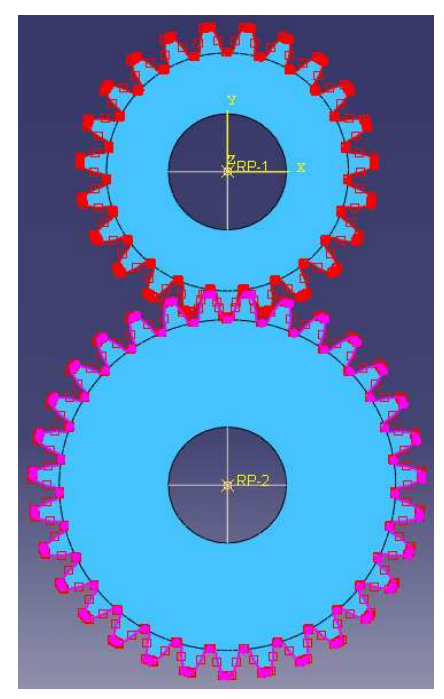

Figure 16: Surface-to-surface contact 


\subsubsection{Small and Finite Sliding}

In addition of determining whether contact has occurred, Abaqus calculates the relative sliding of the two surfaces. According to the Abaqus Theory Manual [13], Abaqus is capable of performing two different type of sliding calculations. One is small sliding, and the other is finite sliding. In small sliding formulation, Abaqus sets up the relationship between the master surface and the slave nodes at the beginning of the simulation, and it determines which segment on the master surface will interact with each node on the slave surface. These relationships will be maintained throughout the simulation, never changing which master surface segments interact with which slave nodes. On the other hand, finite sliding formulation requires Abaqus constantly to determine which part of the master surface is in contact with each slave node. This is a rather complex calculation, especially if both the contacting bodies are deformable. Finite sliding formulation is selected for the gear meshing analysis. Considered the continuous change of contact points on gears teeth as the gears rotate, the never changing condition for a segment of master surface interaction with slave nodes seem to result as conflict. Thus, small sliding formulation appears to be an inappropriate choice for the gear meshing model.

\subsection{FEA Results and Comparisons}

After modeling the gears, assembling, meshing, applying boundary conditions and loads on the system, one should be able to obtain the mesh stiffnesses. Unfortunately, Abaqus is not able to provide the linear tooth mesh stiffness directly from the output displacement field. Therefore, a few extra steps are needed to extract the linear tooth mesh stiffness from the FEA gear model. Since the torque is applied 
on the pinion center node, it makes sense to look at the pinion center node for angular displacement. The applied torque is known, and the angular displacement can be extracted from Abaqus output displacement field. Using equation 4.21, the torsional mesh stiffness $K_{T}$ can be obtained. Furthermore, as previous described (equation 4.23), the linear tooth mesh stiffness $K_{m}$ is related to the torsional mesh stiffness $K_{T}$ through the base circle radius of the pinion $r_{p b}$. The base circle radius of the pinion is known, therefore, the linear tooth mesh stiffness can also be obtained through equation 4.23 .

In addition, the linear tooth mesh stiffness curve will be generated using Microsoft Excel because of its calculating and graphing capabilities. The angular displacements from all the FEA calculations will first be imported into Excel, and then the linear tooth mesh stiffness will be calculated and plotted through Excel functions. Different cases and scenarios will be applied to the gear model, and the results will be compared accordingly. As stated before, the comparisons of linear tooth mesh stiffness will concentrate on the type of elements, the integration methods, the meshing quality, plane stress and plane strain analysis, sensitivity of model tolerance, and crack modeling.

\subsubsection{Type of elements}

In Abaqus, there are extensive element libraries that provide useful tools for solving problems. Users could choose different element families in Abaqus to closely match their problems. One of the major distinctions between different element families is the geometry of the elements. It is because each element family was designed for 
different purposes and applications. For example, shell elements should be applied on structures that contain thin walls, beam elements should be considered when the structures are constructed from beams, rigid elements should only be applied to the structures that are unable to deform, etc. The following figure contains the most commonly used element families in Abaqus.
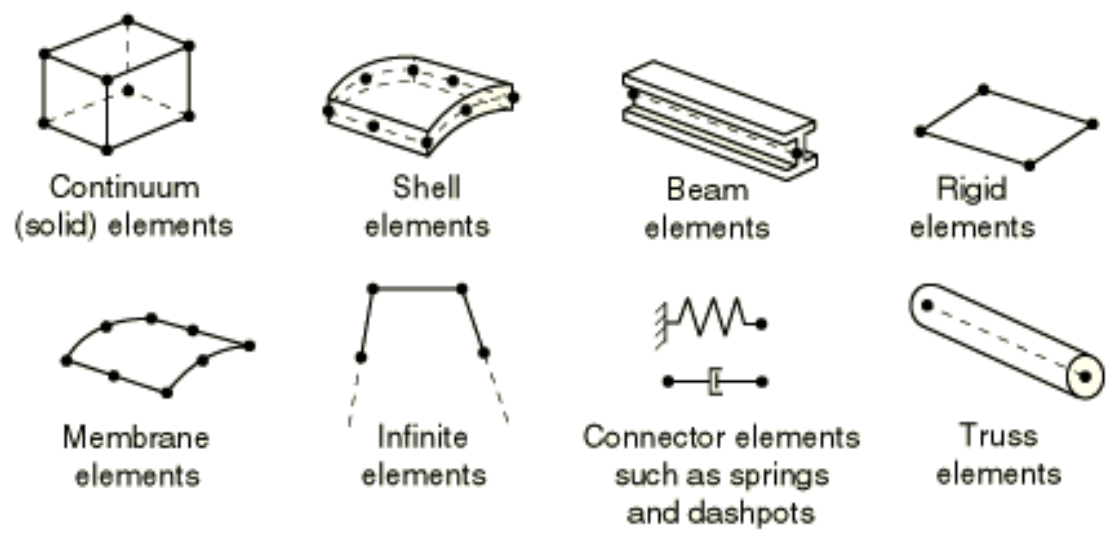

Figure 17: Commonly used element families

(Abaqus Analysis User's Manual [15])

Once the element families have been picked, it is necessary to decide what type of elements should be utilized and its dimensionality. For finite element analysis, the displacements or other degrees of freedom are calculated at the nodes of the element. The displacements are obtained by interpolating from the nodal displacements (equation 4.4), and most often the interpolation order is determined by the number of nodes used in the element. Hence, depending on the number of nodes on the element, different interpolation methods will be applied. The following two figures have demonstrated the node ordering and face numbering on elements, as well as the elements examples with associated names. 


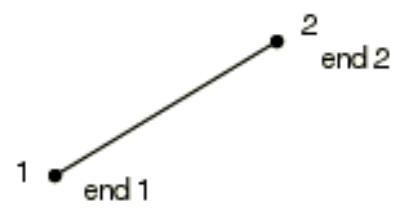

2- node element

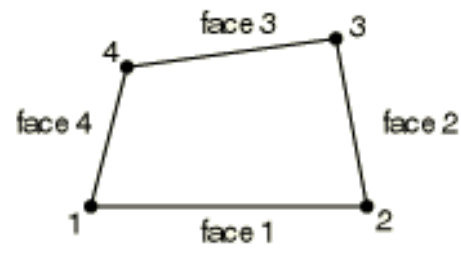

4 - node element

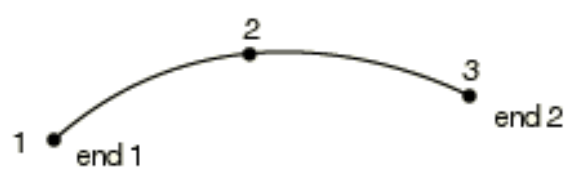

3- node element

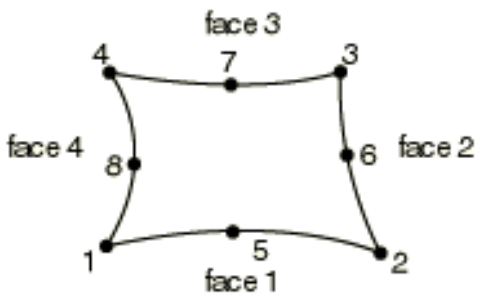

8 - node element

Figure 18: Node ordering and face numbering on elements

(Abaqus Analysis User's Manual [15])

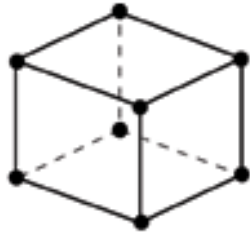

(a) Linear element (8-node brick, C3D8)

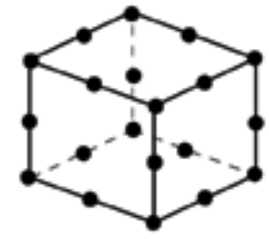

(b) Quadratic element (20-node brick, C3D20)

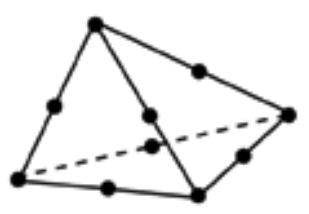

(c) Modified second-order element (10-node tetrahedron, C3D10M)

Figure 19: Solid elements examples

(Abaqus Analysis User's Manual [15])

For the elements that have nodes only at their corners, linear interpolation in each direction will be used. So, these elements will deform linearly, and are known as linear elements or first-order elements. For the elements that have mid-side nodes, quadratic interpolation will be applied to the elements. Therefore, they are often referred to as quadratic elements or second-order elements. According to an online 
source from Curtin University of Technology Australia [14], both quadrilateral (2D) and hexahedral (3D) elements are better suited for solid elasticity modeling as they are the most commonly used elements for non-linear analysis. For a given mesh density with high order, the elements create edges that adapt more closely to curved surfaces than similarly sized linear elements. However, high order elements require much greater computational time and resources.

The first investigation of the gear model studied how the linear tooth mesh stiffness is governed by different elements designation. Two gear models were constructed, one with linear element and the other with quadratic element. The objective is to capture the shape of the linear tooth mesh stiffness curve and to compare the differences between linear and quadratic elements. Figure 20 is the result of the linear tooth mesh stiffnesses generated from Abaqus using linear and quadratic elements.

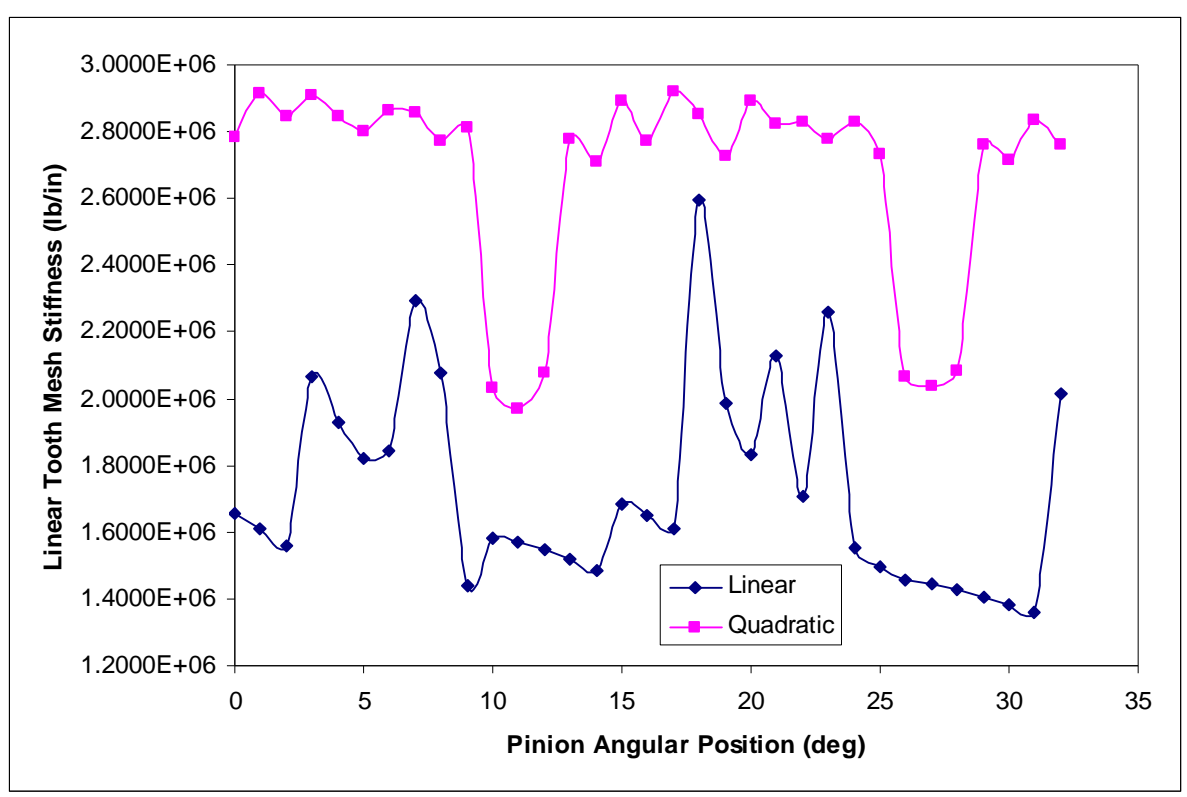

Figure 20: Linear tooth mesh stiffness responses in Linear and Quadratic elements 
From the curves, there is significant different between using linear element and quadratic element. It is rather surprising that linear element would cause such irregularity to the model. This is most likely because linear element can only deform or vary linearly due to its linear interpolation. On the other hand, the quadratic element model has generated a curve that is very similar to those found from previous research [3], [4], and [14]. Also, the computational time of the quadratic elements model does appear to be longer than the linear elements model. Therefore, quadratic element should be used in the gear model for solving linear tooth mesh stiffness.

\subsubsection{Integration methods}

Just like other finite element codes, Abaqus uses numerical techniques to integrate various quantities over the volume of each element. According to the Abaqus Analysis User's Manual [15], Abaqus uses Gaussian quadrature for most elements, and it evaluates the material response at each integration point in each element. Some continuum elements in Abaqus can use full or reduced integration, a choice that can have a significant effect on the accuracy of the element for a given problem. So, should the gear model use reduced integration or full integration? Figure 21 indicates the integration points in the one-dimensional elements. Figure 22 has shown examples of reduced integration and full integration in two-dimensional elements. 


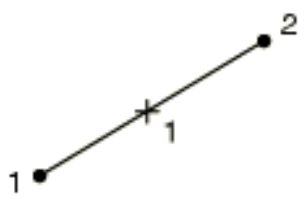

2 - node element

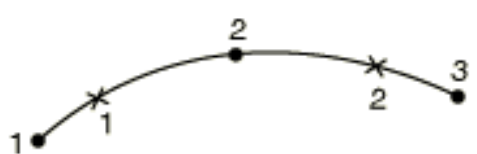

3 - node element

Figure 21: Integration points in element

(Abaqus Analysis User's Manual [15])

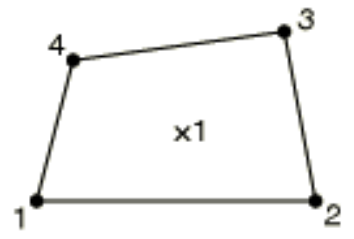

4-node reduced integration element

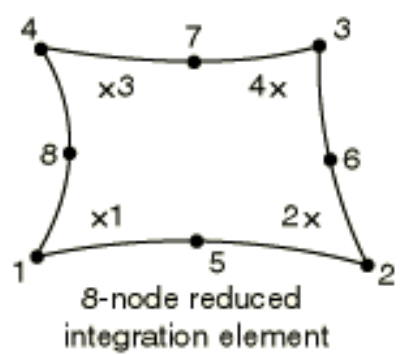

Figure 22: Reduced Integration vs. Full Integration

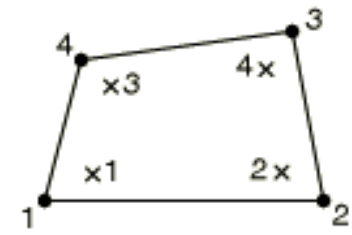

4 - node element

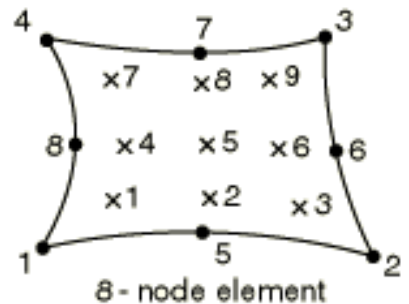

8 - node element integration element

(Abaqus Analysis User's Manual [15])

According to the Abaqus Theory Manual [16], full integration means that Abaqus will integrate the stiffness matrix of an element with uniform material behavior. This means the element sides or faces must be parallel, and the mid-side nodes (for second-order elements) must be at the middle of the element sides. If the element does not satisfy these conditions, full integration is not exact, but such inaccuracy in 
the integration does not appear to be harmful to the element's performance. Also, most fully integrated solid elements are unsuitable for the analysis of incompressible material behavior. It is because the material behavior forces the material to deform without volume changes. However, the number of integration points is sufficient to integrate the virtual work expression exactly, at least for linear material behavior.

Reduced integration means the integration scheme is one order less than the full scheme when integrating the element's internal forces and stiffness. The advantage is that the strain and stress can be calculated at the locations that provide optimal accuracy. Also, the reduced number of integration points decreases the computational time and storage requirements. It also decreases the number of constraints introduced by the elements. However, reduced integration could produce deformation modes that cause no straining at the integration points. This situation could be referred as zero-energy modes which cause a phenomenon called hourglassing, and these zero-energy modes would start propagating through the meshes which lead to inaccurate solutions. To prevent these unwanted deformations, hourglass control procedure should be used. It is a procedure that adds a small artificial stiffness to the element as a precaution against the zero-energy modes.

Instead of explaining the differences between the reduced integration and full integration in words, it would be more beneficial to see how the gear model reacts to these two different integration methods. With that being said, gear models with 
reduced integration and fully integration were created, analyzed, and compared. The following figure shows the model results with different integration methods.

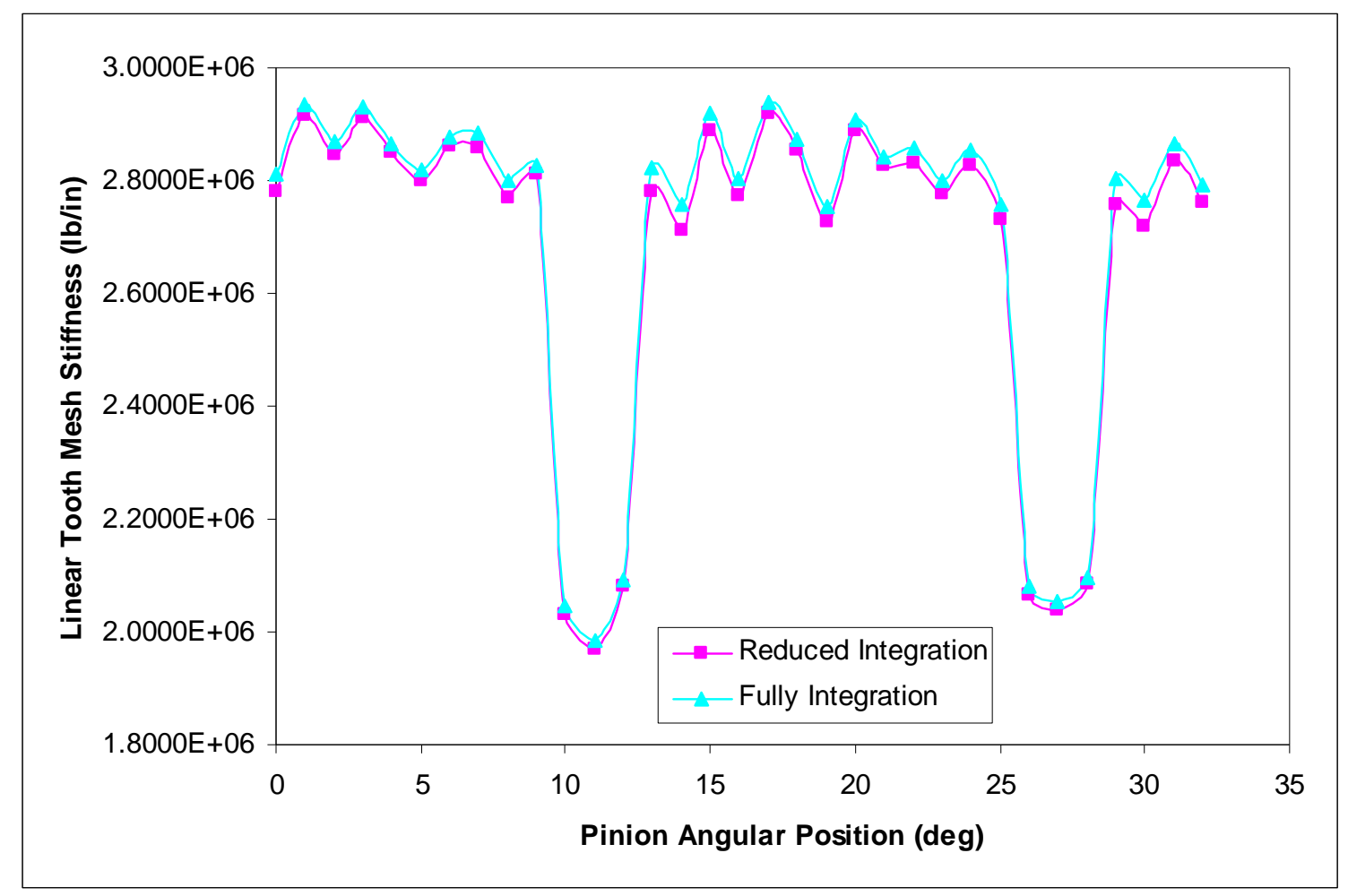

Figure 23: Comparison of gear models with Reduced and Fully Integration

From Figure 23, it is clear that the linear tooth mesh stiffness doesn't fluctuate much between the reduced and fully integration methods. The largest percent difference between the two methods is about $2.5 \%$. This is unexpected because with the complex structure of the gears, one might suspect that the element sides or faces may not be parallel, or the mid-side nodes may not be exactly at the middle of the element sides. Also, the elements' shapes at the gear teeth and around the root of the teeth are rather irregular. This could probably introduce the zero-energy modes that cause hourglassing to the elements which lead to inaccurate solutions. However, none of the above seems to be the case here. In general, because of the reduced number of 
integration points, reduced integration method tend to decrease the computational time when compare to fully integration method. The computational time of the gear model with reduced integration is virtually the same as the one with fully integration. Further investigation is suggested and should be carried out for reduced integration vs. fully integration on the gear model.

\subsubsection{Meshing quality}

The next investigation on the list would be how the meshing quality of the gears affects the linear tooth mesh stiffness. As previous mentioned, once the geometrical model is available, it is necessary to create meshes for analysis process. Sufficient finite element meshes could cause a big impact on the model convergence. Beside the element types, assigning quality meshes (no excessive distortion) also is the essential step to build sufficient finite element meshes. Based on the online source from Curtin University [14], it stated that a good mesh pattern is one that is as coarse as possible on the uninteresting areas, yet as fine as necessary for accurate results where it encounters contact or high stresses. In general, for the gear model, there are three areas that have to be meshed with finer elements. The first one is near the region of contact where the mesh density in the area should be the highest in the gear model. The second area is near the root of the tooth in contact, where the requirement on the mesh density here is at least fine enough to show the correct stress in the area. The third area is around the hub where only a minor refined mesh is required. In Abaqus, users can specify the density of a mesh by creating seeds along the edges of the model to indicate where the nodes of the elements should be located. Also, users can select the shape of the mesh elements. Figure 24 shows a 
model with biased seeding along the top and left edges. Figure 25 displays a model that has been meshed first with quadrilateral elements and then with triangular elements.
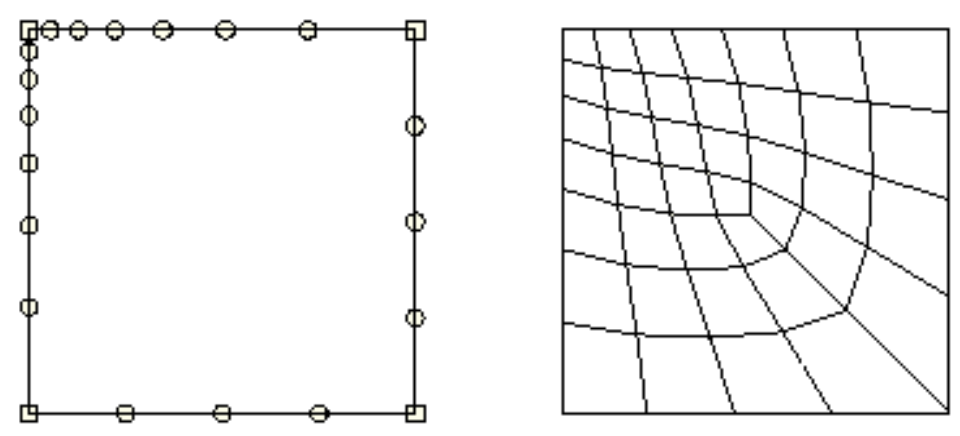

Figure 24: A model with biased seeding (Abaqus/CAE User's Manual [17])
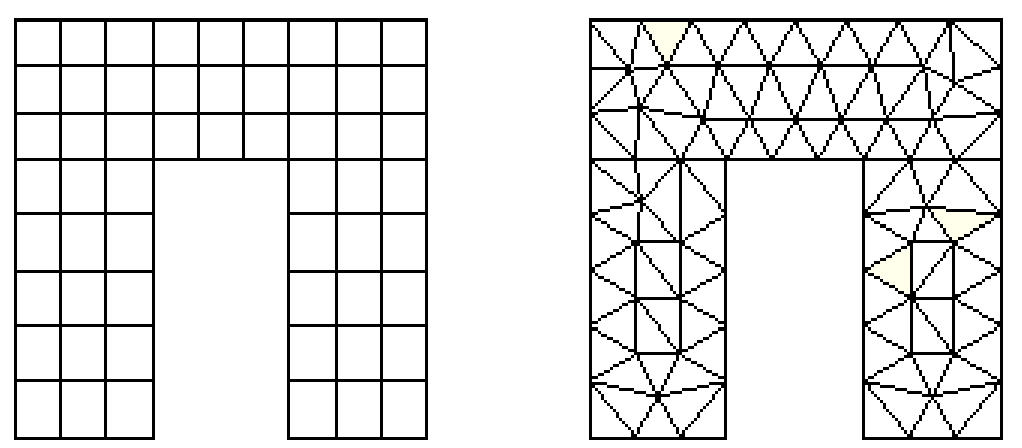

Figure 25: A model meshed with quadrilateral elements and triangular elements (Abaqus/CAE User's Manual [17])

Furthermore, according to Abaqus/CAE User's Manual [17], users can choose the meshing technique - free, structured, or swept. The free meshing technique is the most flexible meshing technique, and it can be applied to almost any model shape. 
However, the tradeoff is that it provides the least control over the mesh since there is no way to predict the mesh pattern. Structured meshing is a technique that provides the most control over the mesh because it applies pre-established mesh patterns to particular model topologies. Users can often partition complex models into simple regions in order to apply structured meshing sufficiently. The swept meshing in Abaqus is created by internally generating the mesh on an edge or face and then sweeping that mesh along a sweep path. Like structured meshing, swept meshing is a technique that limited to models with specific topologies and geometries. In addition to the meshing technique, meshes can be refined using the partition toolset to divide geometric regions into smaller regions. The resulting partitions introduce new edges so that seed can be assigned, which mean users can combine partitioning and seeding to obtain additional control over the mesh refinement. For instance, users can use partitioning to create different regions which are assigned with different element types. For the gear model, coarse mesh and refined mesh have been applied. The objective for applying refined mesh to the gear model is to improve the accuracy of the solutions. The following figure has shown the coarse mesh and refined mesh gear models that generated from Abaqus. 


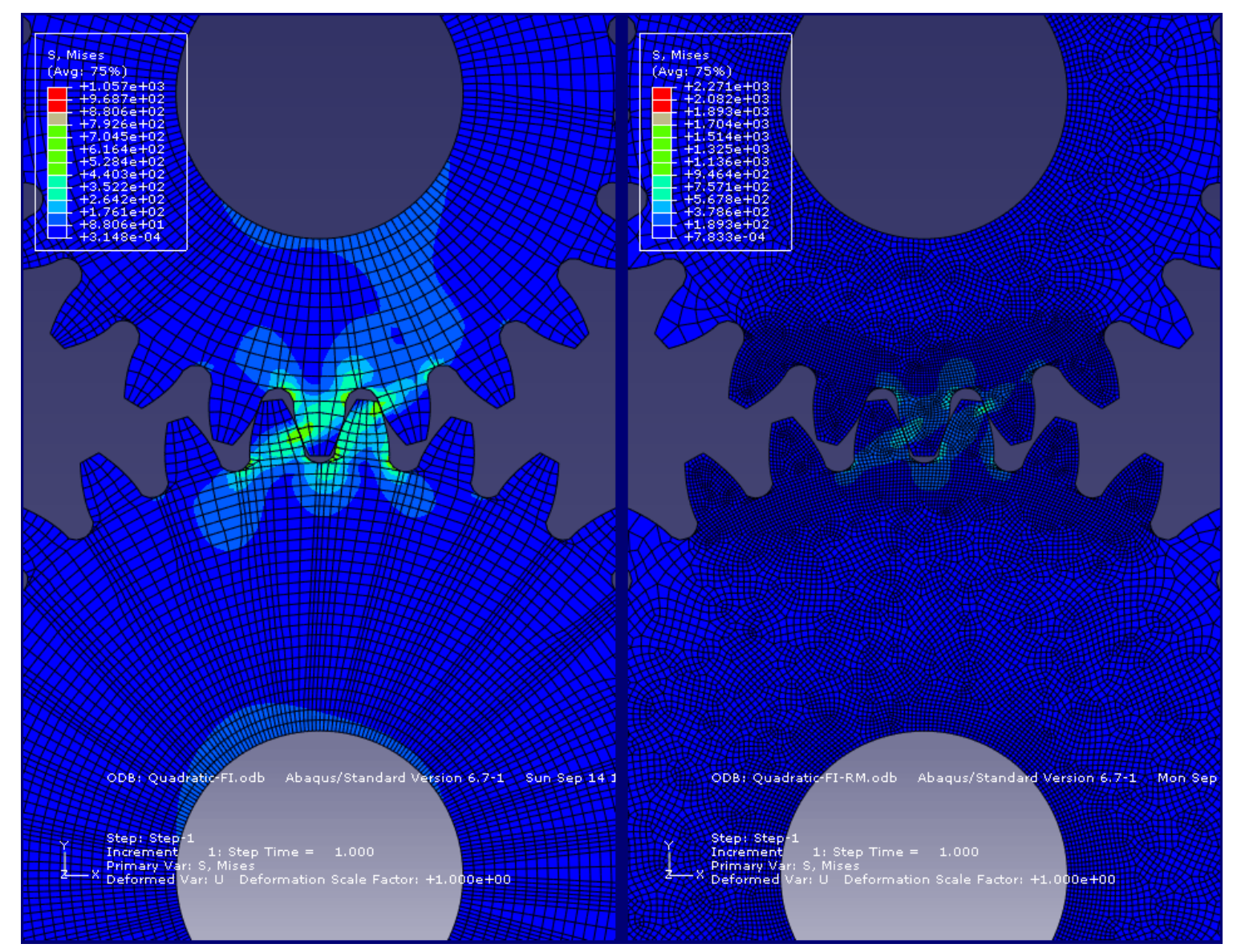

Figure 26: Coarse Mesh vs. Refined Mesh

The left hand side of Figure 26 is a coarse mesh gear model generated using Abaqus built-in auto meshing function. The meshes on the gear teeth and around the hub appear to be sufficient because there are only small amount of distortion in elements. On the other hand, the meshes near the root of the gear teeth contain quite a bit of distortion. Abaqus verifying function is used to verify the element quality. The right hand side of Figure 26 is a gear model that contains a refined mesh in the areas of the gear teeth, near the root of teeth, and of the hub. In order to keep the computational time as short as possible, only few teeth in the pinion and gear are refined (just enough to cover the meshing cycle). The amount of distortion has decreased to 
points mainly located at the root of teeth. The only meshing technique that allowed for the refinement process is the free meshing technique. This is due to the complex structure of the gear model, particularly the gear teeth. As a result, the refined mesh model does have an improvement on the solution.

The figure below shows the changes of linear tooth mesh stiffness as the gear model switching from coarse mesh to refined mesh. The refined mesh curve appears to be less fluctuating at the double teeth contact zone, but it still contains some disturbance. Also, at the one tooth contact zone (between $9^{\circ}$ to $13^{\circ}$ ), the refined mesh model appears to be narrower in the range. This could be due to the contact tolerance (will be discussed in later section) between the pinion teeth and the gear teeth. Since the refined mesh model still contains distorted elements around the root of the teeth, additional mesh refinement in this area should be considered in hope of further improving the solution. 


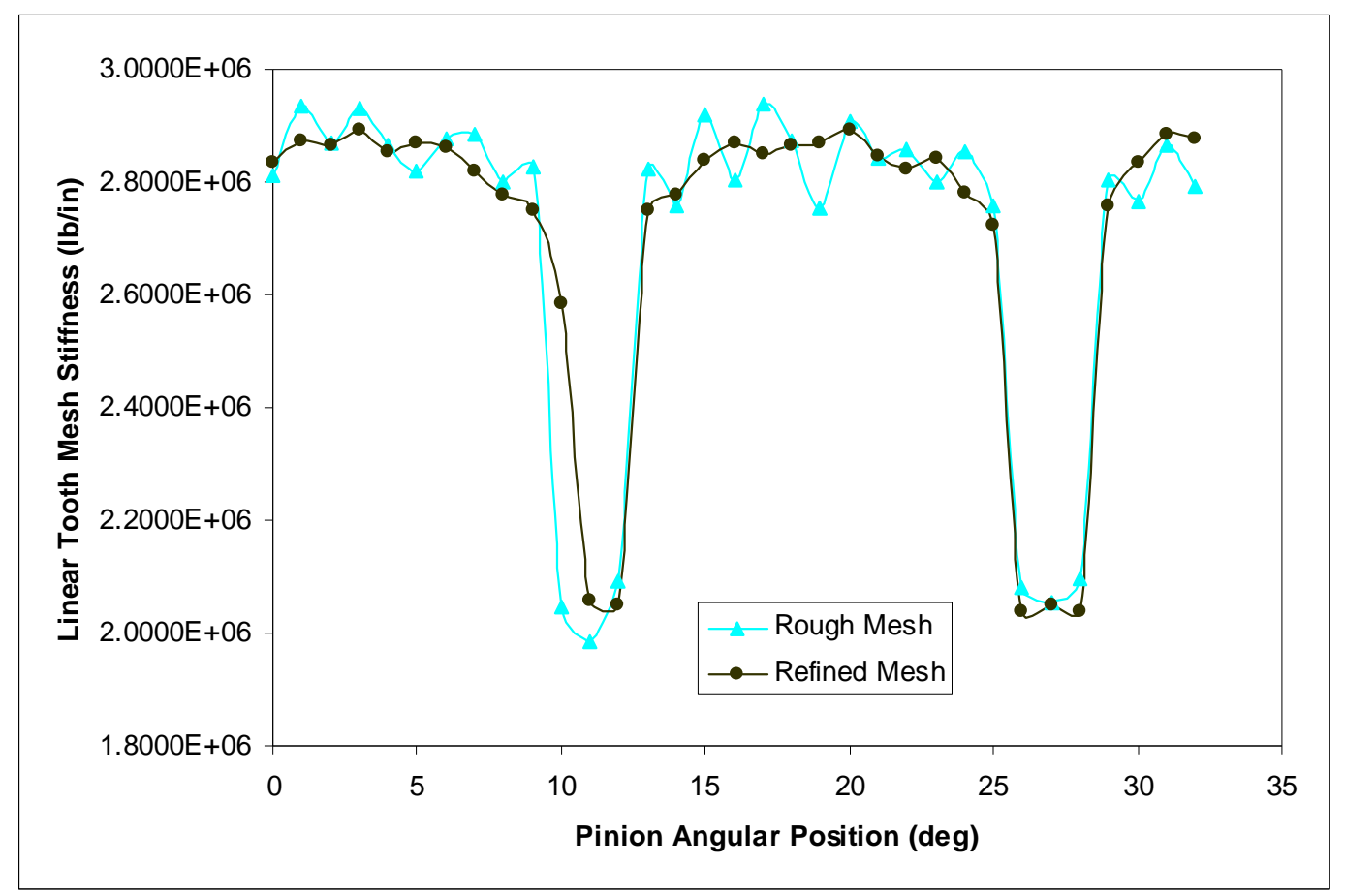

Figure 27: Linear tooth mesh stiffness changes with meshing quality

\subsubsection{Plane Stress and Plane Strain analysis}

In general, the stresses near the contact areas and the root of teeth are three dimensional for gears in mesh. However, most previous research on FEA gear models [3], [4], [6], [14] with standard involute gears have reduced the problem to two dimensional, and many of them provide acceptable approximations. For two dimensional models, one of the three principal stresses or strains is assumed to be zero, which resulted in the model to be either plane stress or plane strain. For plane stress or plane strain, one dimension is significantly different when compared to the other two. So, the structure usually appeared to be either thin or thick. Then, for gear modeling, the question raises as to should the plane stress or the plane strain assumption be used in the gear models. It is not known if the plane stress or the plane strain assumption is the most appropriate. Nevertheless, as stated from Arthur \& 
Chong [18] and Richards [19], a general guide which is supported by elasticity theory, for FE modeling of a thin plate with in-plane loads and boundary conditions, the plane stress assumption should be used regardless of whether the solutions are for displacements or for stress.

In the plane stress state, stresses are negligible in the smaller dimension since they are not able to develop within the material. Therefore, the stresses are functions of planar coordinates alone, and the out-of-plane normal and shear stresses are equal to zero. In Abaqus, for plane stress, the elements must be defined in the $X-Y$ plane; all loading and deformation are also restricted to this plane. This modeling method generally applies when the thickness of a body or domain is small relative to its lateral (in-plane) dimensions. So, the structural element can be analyzed as two dimensional thin-walled models. In contrast, at plane strain state, the strains in the direction of the longest dimension can be neglected. Therefore, the strains in a loaded body or domain are functions of planar coordinates, and the out-of-plane normal and shear strains are equal to zero. Similarly, the elements must be defined in the $X-Y$ plane, and all loading and deformation are also restricted to this plane. Plane strain is generally used for bodies that are very thick relative to their lateral dimensions. So, it indicates that the structural element can be analyzed as two dimensional thick models such as shafts, concrete dams, or walls.

Two separate gear models were built in Abaqus, one with plane stress elements while the other with plane strain elements. In Abaqus, for homogeneous solid 
sections, it requires users to provide a Plane stress/strain thickness which is going to be used as the thickness of the two dimensional region. For two dimensional problems, users must specify the section thickness in order for Abaqus to initiate the analysis process. Hence, in the gear model case, this Plane stress/strain thickness will serve as the thickness of the gears. For simplicity, all the gear models were considered with the thickness of 1 inch. The main motivation for choosing a thickness of 1 inch is to merely normalize this value on a per unit basis. It would be obvious that plane stress elements would generate better result if the thickness of the gear is very thin, likewise, plane strain elements would produce better result if the thickness is very thick. It is hard to judge if it is thin or thick for the gear with 1 inch thickness, which make it an excellent test subject. The following are the results of the linear tooth mesh stiffness using plane stress and plane strain elements with 1 inch thick gears.

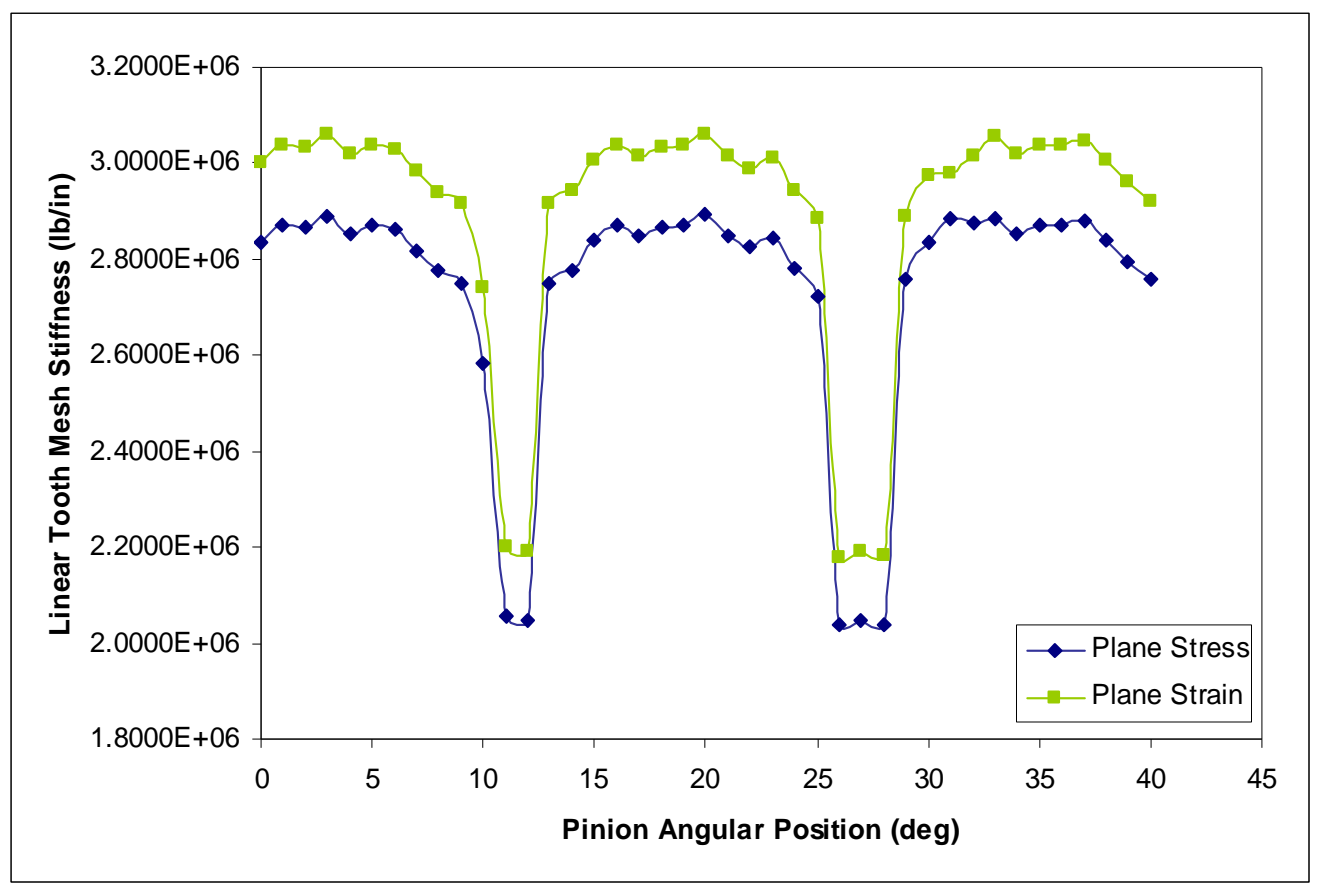

Figure 28: Plane Stress vs. Plane Strain 
From Figure 28, the shapes of the curves are virtually the same, but it is clear that using plane strain elements would result in higher stiffness. The percent difference between the two curves is about $7 \%$. So, how would one know which element is a better suit for analysis? The answer to that is to compare the FEA results to a theoretical solution or a closed-form solution. A theoretical gear meshing model was derived by Dr. Meagher [20], and was used to compare the result of linear tooth mesh stiffness. The result of comparison can be found in the later chapter. Furthermore, from previous chapter, a simple mesh stiffness calculation with basic cantilever beam theory was performed. This calculation was operated as closed-form solution, and was used to compare with FEA results. As a result, the FEA mesh stiffness values with plane stress elements were closer to those from the theoretical model and closed-form solution. Hence, although plane stress and plane strain elements generate similarly shaped stiffness curves, it seems plane stress elements should be used in finite element process since it produced slightly better magnitude results when compare to the theoretical model and closed-form solution.

\subsubsection{Sensitivity of model tolerance}

Almost in every designed part, tolerance always plays a role in the design and manufacturing process. This role becomes particularly important when a high quality part is desired. However, this high quality desire does come with a price. In general, manufacturing a tight tolerance part would increase the manufacturing time, thus, the production cost would increase as well. Just like manufactured part, the finite element gear model also has an issue of tolerance when it comes to contact. The main issue about the model is determining whether the gear teeth actually in contact. 
After the contact surfaces and contact interactions are defined, Abaqus assumes that the surfaces are already in contact at the assembled stage. If the surfaces are not in contact at the beginning of the analysis, Abaqus/Standard will not be able to calculate the result. It is because of the contact constraints; Abaqus tries to solve the system as a contact problem even though the surfaces are not in contact. Therefore, a tolerance is needed in order for Abaqus to initiate the correct analysis. This tolerance indicates that the surfaces are considered in contact if they are within this tolerance value. Ideally, this value should be zero which mean the surfaces are in contact at the assembled stage. Hence, this tolerance value should be as small as possible. Furthermore, the tolerance value can be entered in the Interaction Module, under the Slave Node/Surface Adjustment tap. The following figure is an example of entering the tolerance value.

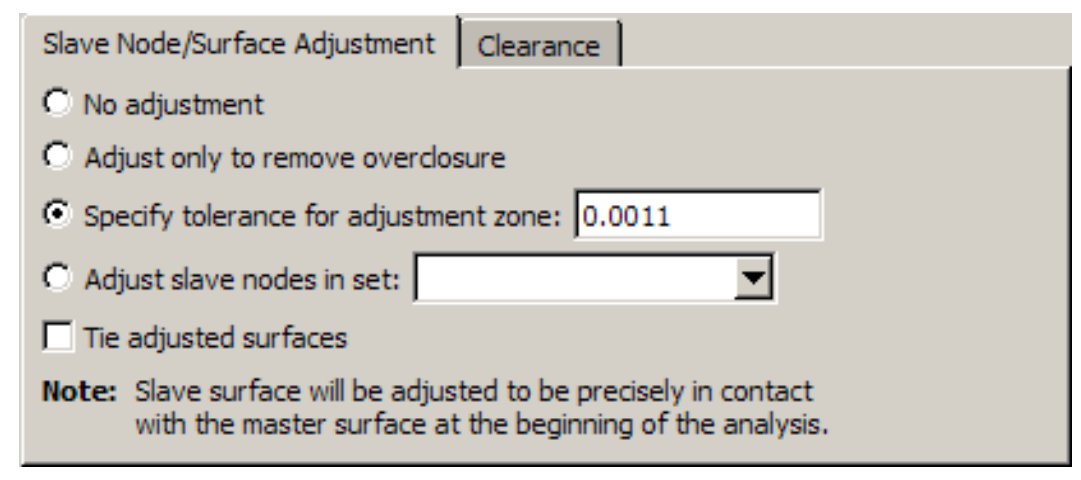

Figure 29: Specify tolerance value of the model

A range of tolerance values are selected and applied to the gear model. Figure 30 shows the linear tooth mesh stiffness response to the different tolerance values. From the graph, it is clear that when the tolerance value is too small or too large, the results 
are poor. One interesting point to note is that as the tolerance value increase, the single tooth contact zone is getting narrower.

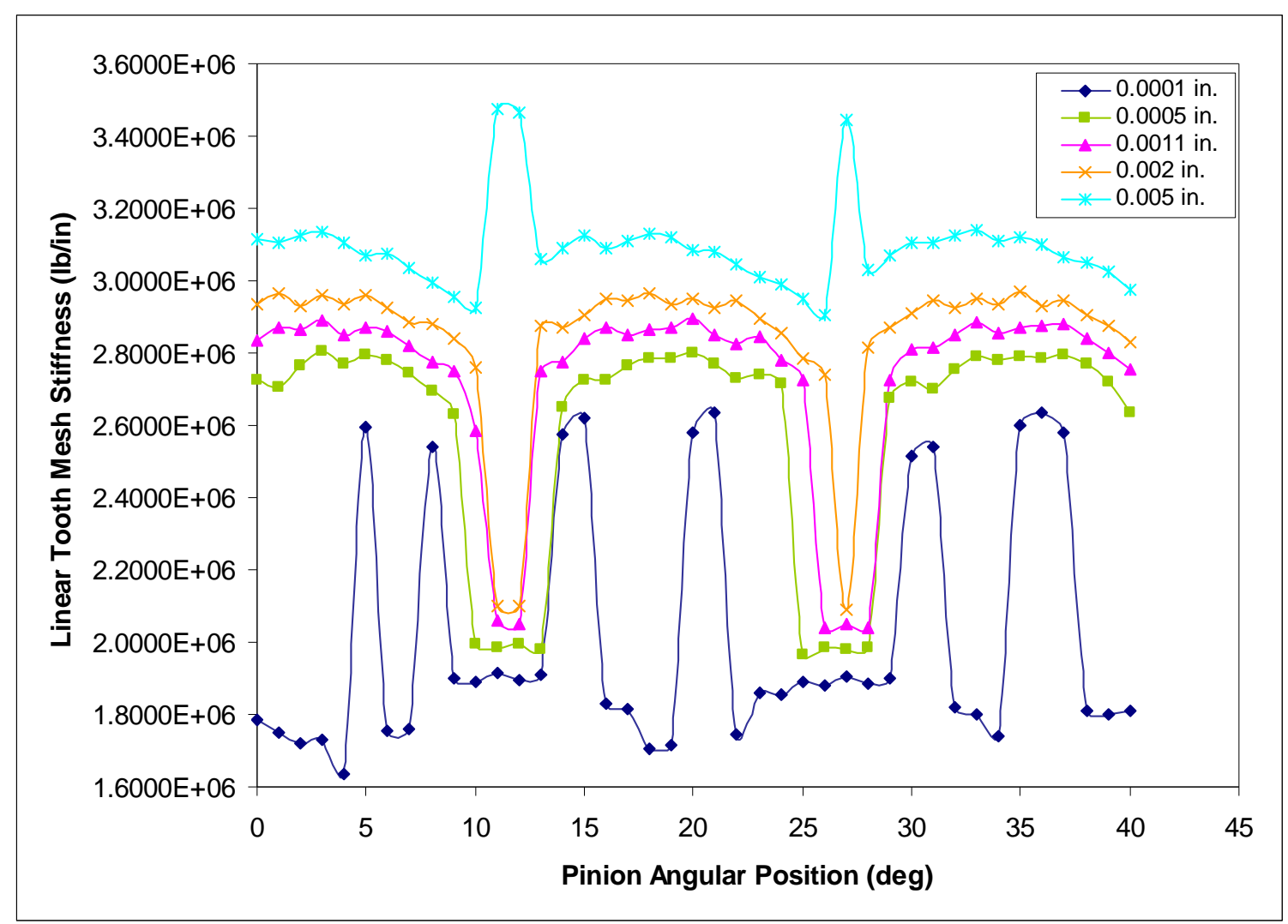

Figure 30: Mesh stiffness response to different tolerance values

\subsubsection{Crack modeling}

One of the major concerns or topics in studying gear transmission systems is the behavior of the faulty gears. Faulty gears means gears that are defected, no longer perfect, or in a condition that is far from its original designed shape. It also means the gear can no longer maintain or function at its full potential. Furthermore, faulty gears consist of various gear conditions such as a cracked tooth, chipped tooth, missing tooth, wear and tear, etc. These various gear conditions could be achieved unintentionally or intentionally. Gears that reach these conditions with time through 
fatigue are considered unintentional. Obviously, gears that reach these conditions through machining or manufacturing are regarded as intentional. The study of faulty gears is important because failure to notice or identify faulty gears in systems could result in catastrophic malfunctions or accidents. It would take an extremely long duration and large amount of resources to fully investigate all the scenarios of faulty gears. Therefore, in this research, only a cracked tooth will be considered. Regarding cracked gears, fracture mechanics has been used to study and predict crack propagation. From previous work Ural et al. [21], predicting crack trajectories is important in determining the failure mode of a gear. Cracks propagating through the rim may result in catastrophic failure, whereas the gear may remain intact if one tooth fails. This may allow for early detection of failure. Although it is important to examine crack propagation on gear teeth, the scope of this research will focus on how the cracked tooth affects the linear tooth mesh stiffness.

FEA gear models with a cracked tooth were built and analyzed. The cracked tooth was analyzed under two different categories. One category is the location of the crack, and the other is the length of the crack. As the gears rotate through the meshing cycle, the impact points on the gears' teeth are constantly changing. So, what locations should the crack be? Based on previous work [3] and [6], the crack locations are focused around the pitch circle and at the root of tooth. These are logical choices because when the gears are rotating, the pitch circles of the gears will rotate without slipping, hence, there will always be stresses around the pitch circles area. Also, as the gears rotate, the gears' teeth are acting like short cantilever beams 
to some extent. So, the stresses would build up at the root of the teeth just like stresses would build up at the end of a cantilever beam. For the length of the crack, 0.01 inch and 0.03 inch will be the chosen length of the crack. There are no particular reasons why these lengths were chosen. The purpose is just to observe how the change of length would affect the mesh stiffness curve. Figure 31 demonstrates the locations of the crack and the length of the crack in the finite element gear models. Figure 32 is the results of the cracked tooth gear models with different crack lengths at different locations of the tooth.

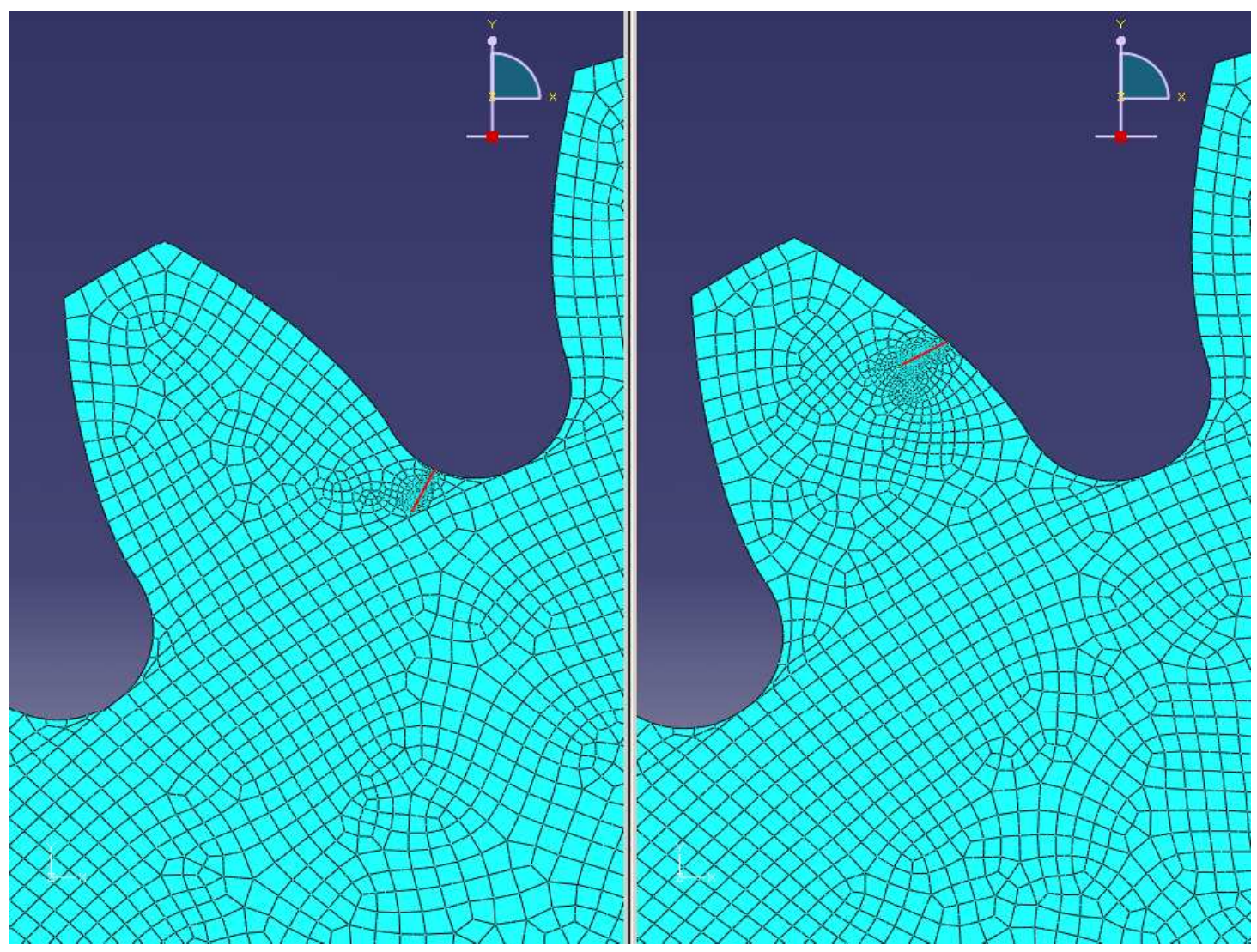

Figure 31: Different crack locations 


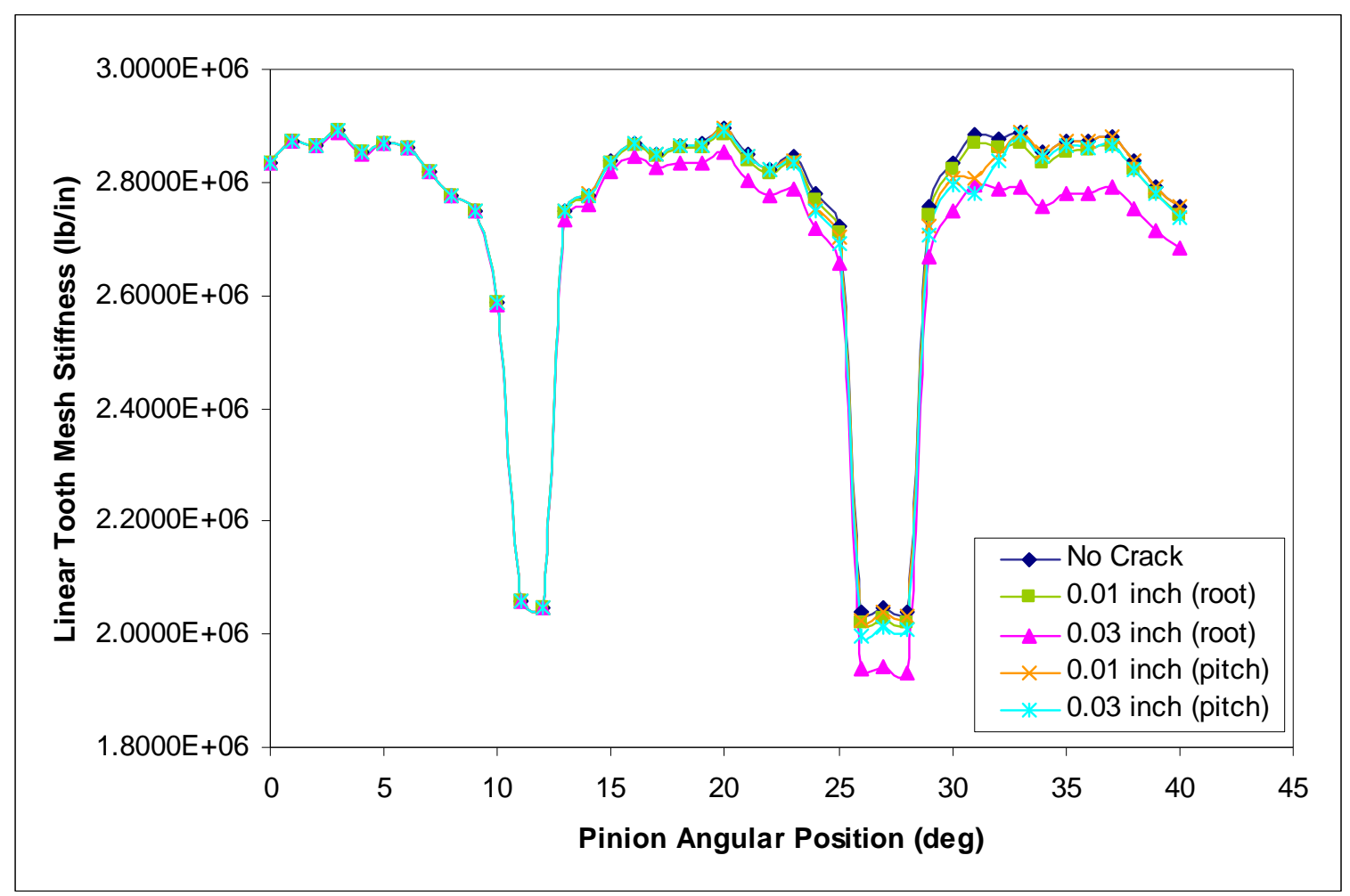

Figure 32: Mesh stiffness result with various crack scenarios

As one can see from Figure 32, the linear tooth mesh stiffness has decreased over the cracked tooth region. This is expected because the crack has created a stress concentration over the area which ultimately softens the system as the load was applied. From these results, the lengths of the crack affect the mesh stiffness more at the root of the tooth rather than at the pitch circle of the gear. The change in crack length at the pitch circle only displays a small change in the mesh stiffness. This shows that the gear model is more responsive to the crack that is at the root of the tooth than at the pitch circle. Furthermore, even though a long crack has generated a more visible change than a short crack, it is just as important to investigate short 
cracks as well. In a way, a short crack is more essential from a diagnostic stand point since problems or defects should be detected before the tooth fracture.

\subsection{Verifying Results}

As a common rule, FEA results such as displacements, stresses, reaction forces, etc, should be compared with closed-form calculation and experimental testing in order to evaluate if the numerical solutions correlate with the response of the physical structure. For the gear model case, measuring mesh stiffness experimentally would be challenging since the gearbox has very limited space for setting up sensors or other apparatus. On the other hand, comparing the FEA mesh stiffness with closedform calculation is a much easier approach. Using simple cantilever beam theory, the mesh stiffness can be estimated without any computational power. Therefore, the closed-form calculation will be used to compare with the FEA results. The results of the stiffness comparison can be found in the conclusion chapter. 


\section{Experiment}

\subsection{Overview}

In many industries, gear transmission systems are important; hence, the knowledge and understanding of the behavior of gears in mesh becomes essential. Experimental testing is a great way to diagnose vibrations in a gearbox. In another words, the main purpose of the gearbox experiment is to measure and identify vibration frequencies that are associated with the gears and the bearings in a gearbox. The typical methods used in frequencies monitoring are utilizing case mounted accelerometers. The selected gearbox is manufactured by Spectra Quest, and it is called the Gearbox Dynamics Simulator (GDS). GDS is an experimental apparatus that is used for studying and researching gear phenomenon and performance. Different applications could be applied either separately or simultaneously on the GDS. This is a useful tool for introducing gear concepts to engineering students. Furthermore, GDS is not an off-the-shelf gearbox; Spectra Quest has built and assembled the GDS using high tolerances so that it would not be affected by undesired vibration. The following is a list of typical applications that can be applied on the GDS.

1. Modal Analysis and Mode Shapes

2. Crack shaft analysis

3. Analysis on Chipped, Broken, or Cracked gear teeth

4. Gearmesh and Bearing Frequencies

5. $\quad$ Effects of bearing type

6. $\quad$ Loading effects (applying brake) 
7. Misalignment and Backlash studies

\subsection{Frequencies Calculations}

Before any calculations are performed, it is necessary to decide the type of gears and bearings that are used in the GDS. There are two sets of gears in the GDS, and both sets are spur gears. The first stage consists of pinion with 24 teeth and gear with 60 teeth; the second stage consists of pinion with 36 teeth and gear with 48 teeth. The bearings used in the GDS are rolling element bearings. In addition, the rotation speed or the input shaft speed is also required for the frequencies calculation. Two different rotation speeds are chosen for the experiment: $17 \mathrm{~Hz}$ and $25 \mathrm{~Hz}$.

\subsubsection{Gear Frequencies Calculations}

Gear Ratio:

$$
g_{n}=\frac{N_{p}}{N_{g}}
$$

Gearmesh Frequency:

$$
G M F=N_{p} \cdot \Omega_{p}=N_{g} \cdot \Omega_{g}
$$

Fractional Gearmesh Frequency:

$$
F G M F=\frac{n}{C F} \cdot G M F
$$

where $n=1,2 \ldots$ and $C F=$ common factor

Hunting Tooth Frequency:

$$
H T F=\frac{\Omega_{p}}{U F_{g}}=\frac{\Omega_{g}}{U F_{p}}
$$


where, $N_{p}$ and $N_{g}$ are the number of teeth of pinion and gear respectively

$\Omega_{p}$ and $\Omega_{g}$ are the rotation speeds of the pinion and gear respectively $U F_{g}$ and $U F_{p}$ are the uncommon factors of the gear and pinion respectively

\subsubsection{Bearing Frequencies Calculations}

Number of Elements, $N_{e}$ : 16

Shaft Rotation Speed, $\Omega$ : $\quad 17 \mathrm{~Hz}$ and $25 \mathrm{~Hz}$

Pitch Diameter, $D$ : $\quad 1.5237$ inch

Element Diameter, $d$ : $\quad 0.225$ inch

Element Contact Angle, $\alpha: \quad 10^{\circ}$

Outer Race Ball Pass Frequency:

$$
O R B P=\frac{N_{e} \Omega}{2}\left(1-\frac{d}{D} \cos \alpha\right)
$$

Inner Race Ball Pass Frequency:

$$
I R B P=\frac{N_{e} \Omega}{2}\left(1+\frac{d}{D} \cos \alpha\right)
$$

Fundamental Train Frequency:

$$
F T F=\frac{\Omega}{2}\left(1-\frac{d}{D} \cos \alpha\right)
$$

Ball Spin Frequency:

$$
B S F=\frac{D \Omega}{2 d}\left(1-\left(\frac{d}{D} \cos \alpha\right)^{2}\right)
$$




\subsection{Features of the GDS}

The GDS is a parallel shaft gearbox with a braking mechanism. It consists of the following components:

1. Motor drive

2. Speed control interface box

3. Optical speed sensor

4. Parallel shaft gearbox

5. $\quad$ Braking mechanism (with controller)

\subsubsection{Motor drive}

The motor is 3 horsepower, 2-pole, and 3-phase. The speed range for this motor is 0 to $3,600 \mathrm{rpm}$. A $1 / 2$ in plate is used for the motor support, and it is fixed to the motor by hex screws. In order to align the motor and the gearbox shaft, the plate is used to elevate the motor.

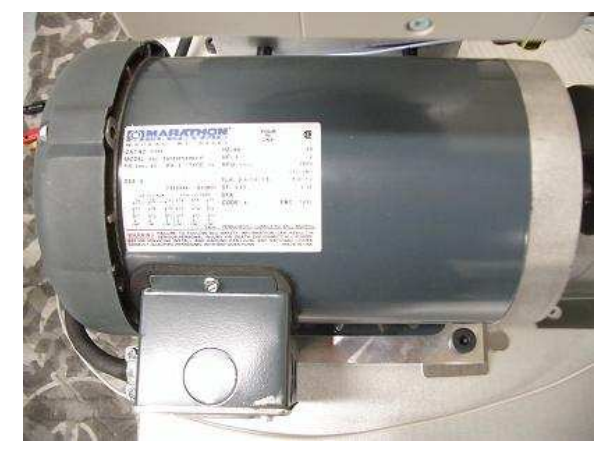

Figure 33: $3 \mathrm{hp}, 2$-pole, 3-phase Motor

\subsubsection{Speed control interface box}

VFD-B series speed control box from Delta Electronics Inc. is used to control variable speed of the 3-phase motors. This component contains different parameter 
settings for user. Based on the applications need, users can set the desire parameter before or during operation.

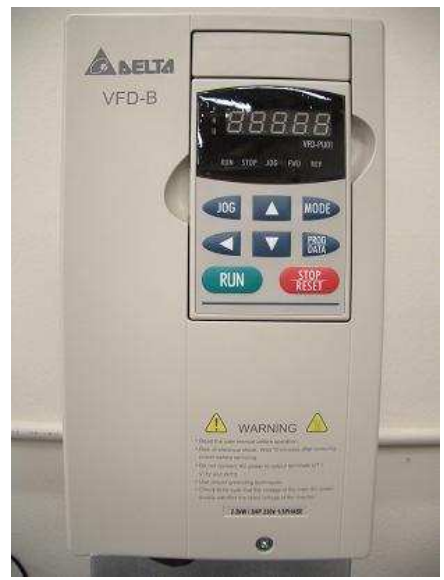

Figure 34: Delta VFD-B Speed Controller

\subsubsection{Optical speed sensor}

A non-polarized retro-reflective mode senor from Banner will be used to indicate the input shaft speed. A reflective tape will be placed on the motor shaft, and the sensor will be aligned so that it is perpendicular to the reflective tape. Also, the sensor will be connected to the Red Lion MODEL CUB5 digital counter/rate indicator which is used to display the input shaft speed.

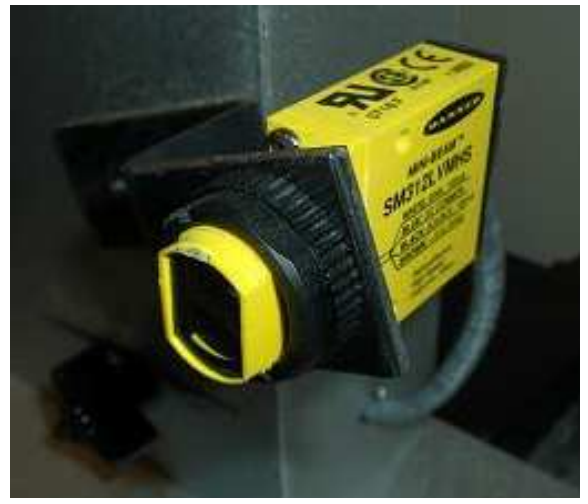

Figure 35: Banner Optical Speed Sensor

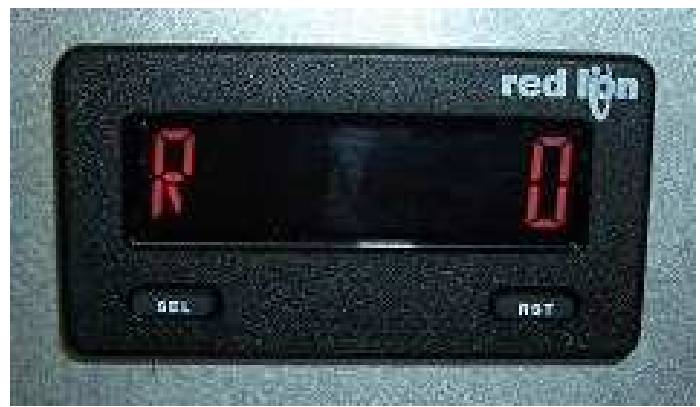

Figure 36: CUB5 digital counter 


\subsubsection{Parallel shaft gearbox}

There are three shafts in the gearbox, and they are all parallel to each other. The gears can be slid along the length of the shafts; spur or helical gears maybe fitted for desired gear cases. There are number of 1/4-28 threaded holes on the sides and top of the gearbox for proximity probes which will be placed at 90 degrees to the shafts. However, the distance between the threaded holes and the shafts is too great for standard proximity probes. So, a special bracket is required to extend the reach of the proximity probes. The gearbox is filled with SAE $80 \mathrm{~W}-90$ lubricant in order to minimize gears wear.

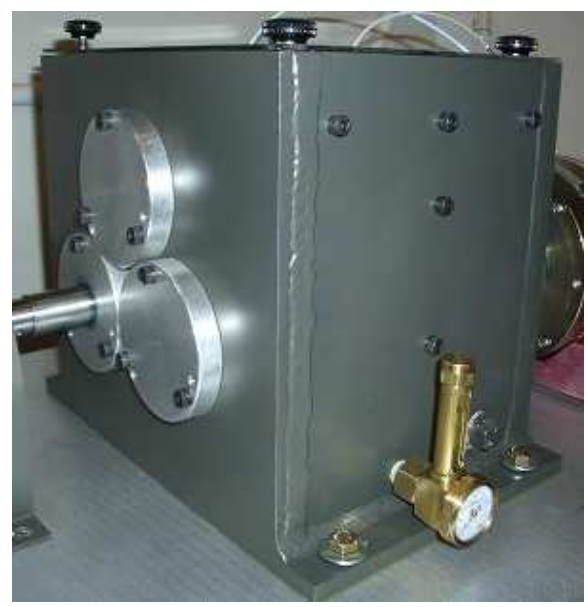

Figure 37: Gearbox Housing

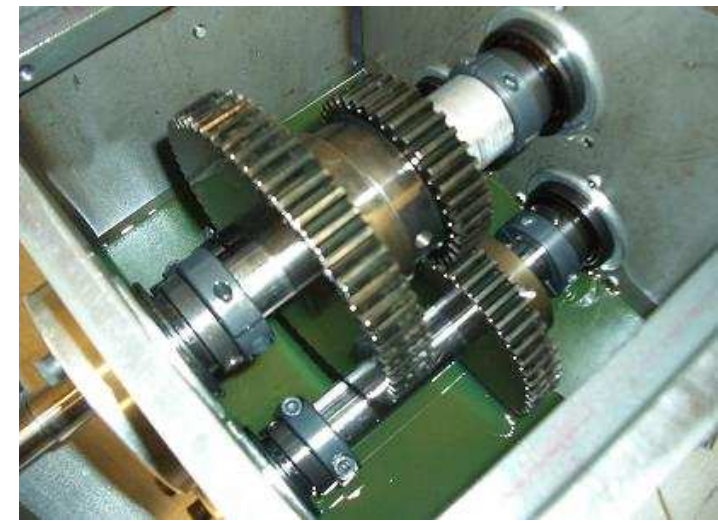

Figure 38: Parallel Shaft with Spur Gears

\subsubsection{Braking mechanism}

The output shaft of the gearbox will be connected to a DIGI-Series Power Supply from Electro Industries Inc. The power supply acts like a brake and provides torsional loading on the system. The torsional load can be varied by changing the voltage or current of the power supply. So, greater vibration amplitude can be achieved by increasing load which will cause greater gear defection as well. 


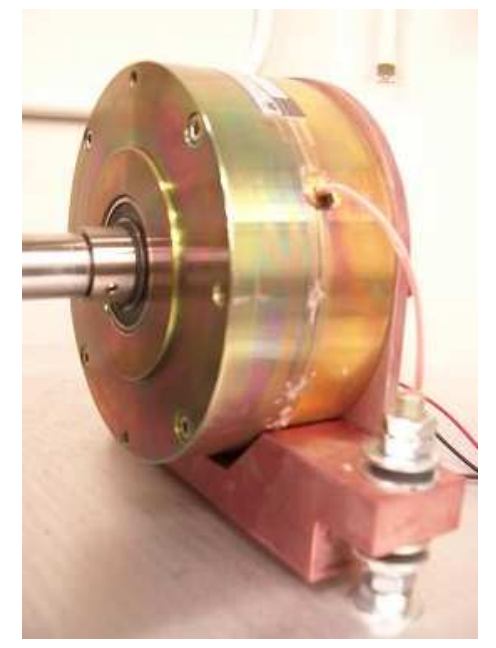

Figure 39: Loading Mechanism (Brake)

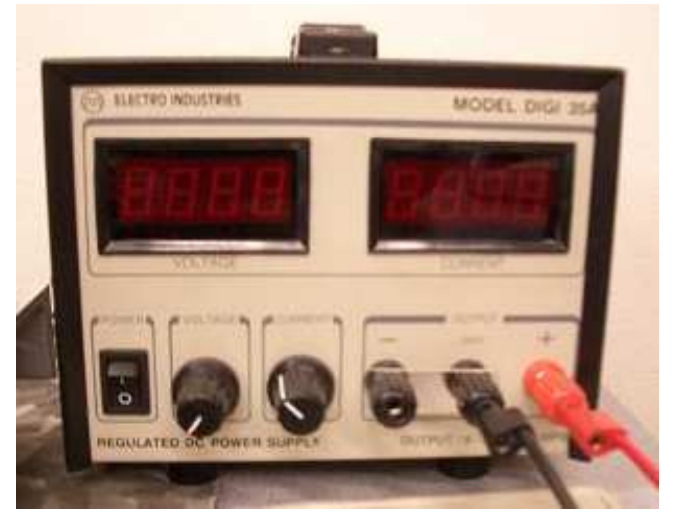

Figure 40: Brake Controller

\subsection{Experimental Setup}

\subsubsection{Gearbox Dynamics Simulator}

First, the GDS unit needs to be assembled. The following schematic shows the major components of the simulator, and it illustrates how each component should be placed and assembled. (For detail information about assembling the GDS, please read the Spectra Quest GDS Operating Manual) 


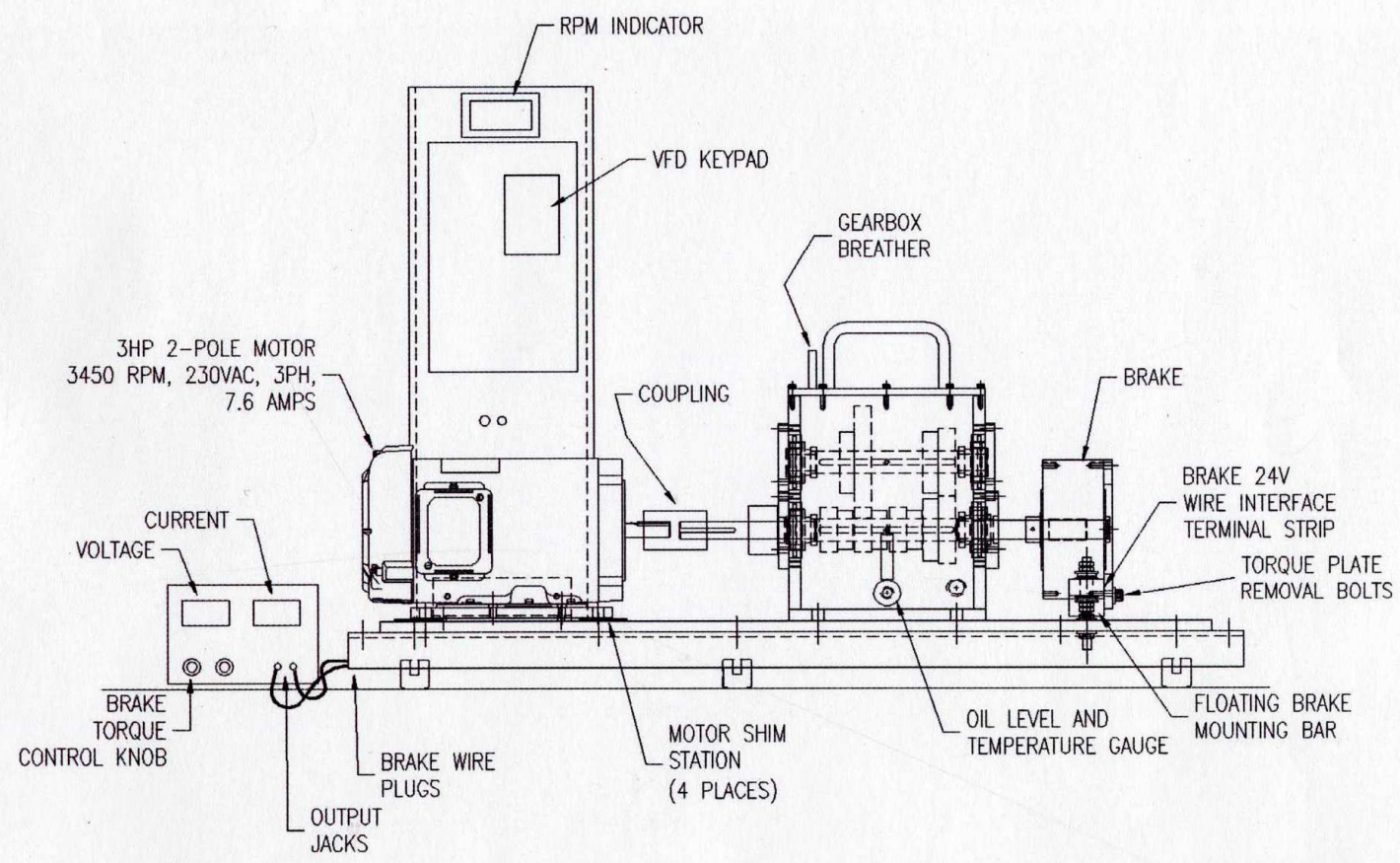

Figure 41: Schematic of the GDS

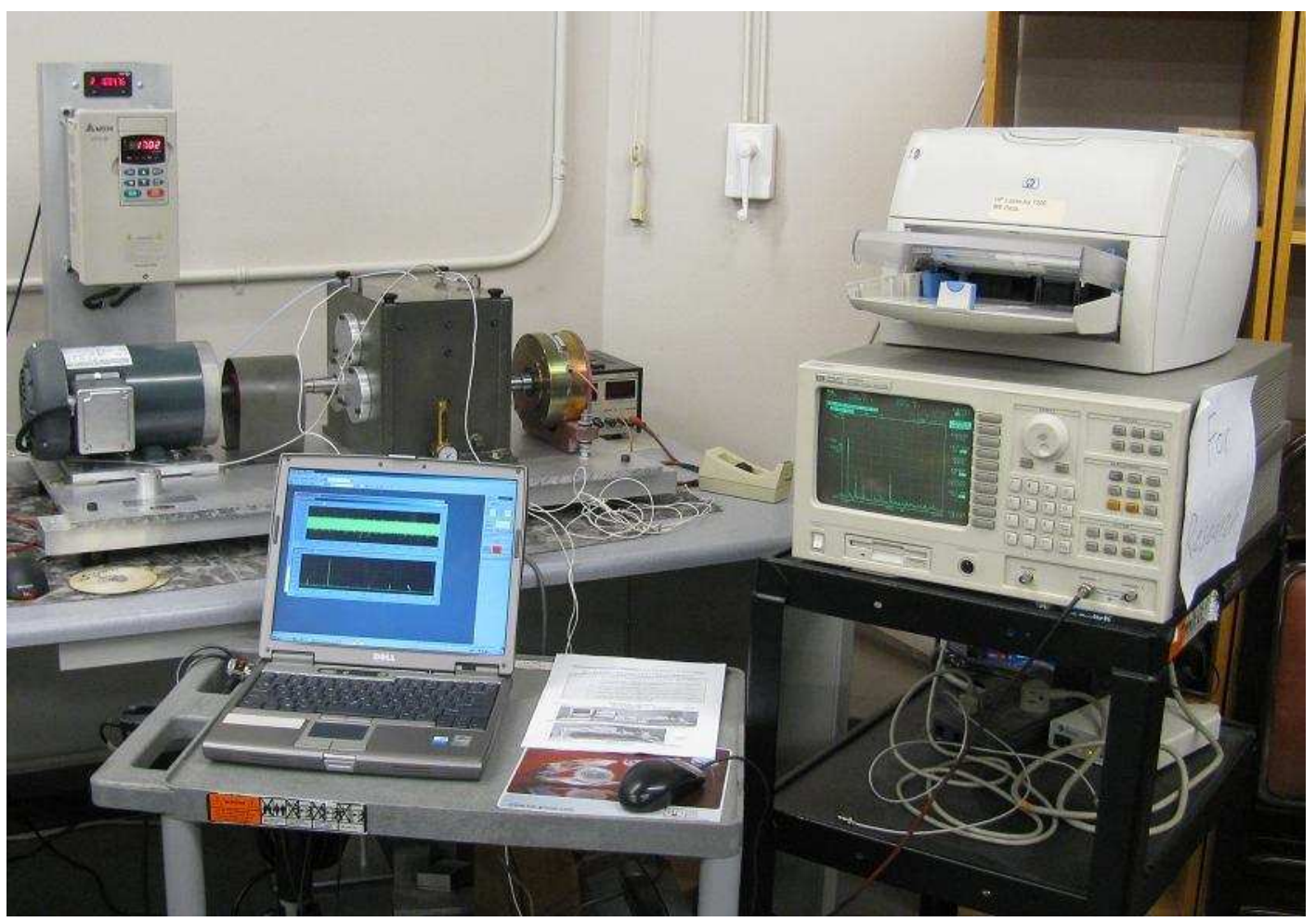

Figure 42: GDS Setup with ME'scopeVES and spectrum analyzer 


\subsubsection{Bently Nevada Equipment}

ADRE Data Acquisition (Figure 43) from Bently Nevada will be set up and run in conjunction with the GDS. The objective is to measure the imbalance of the rotor, and to obtain orbital plots of the shaft at different running speeds. In order for the ADRE system to work, keyphasor and proximity probes are necessary. The gearbox has built-in threaded holes for proximity probes, but the threaded holes are too far from the shaft. Therefore, in order to use standard proximity probes, it is necessary to manufacture a custom bracket that could extend the reach of the probes (Figure 44). The bracket has been designed using SolidWorks and manufactured at the Cal Poly machine shop. Gears will be slid on the shafts in the gearbox. Please note that the key slot on the shaft will interfere with the probes reading. Thus, an adjustable hub will be used to cover the exposed key slot so that reading can be obtained (Figure 45). Same as the bracket, the hub has been designed in SolidWorks and manufactured at the Cal Poly machine shop. Keyphasor is an important component in ADRE system; it is a trigger that resulted from a point on a rotating shaft. It basically serves as a phase reference for determining where imbalance is on a rotor. The Optical Speed Sensor described in section 5.3.3 also serves as a keyphasor in the system. 


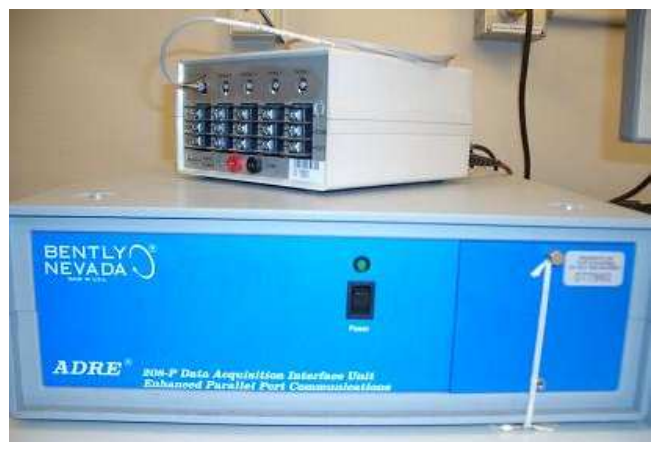

Figure 43: ADRE Data Acquisition

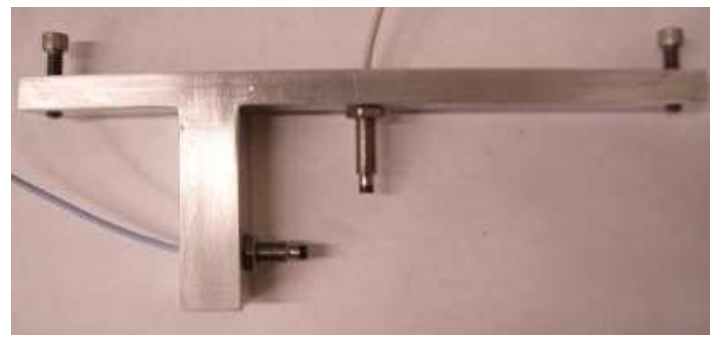

Figure 44: Proximity Probes Bracket

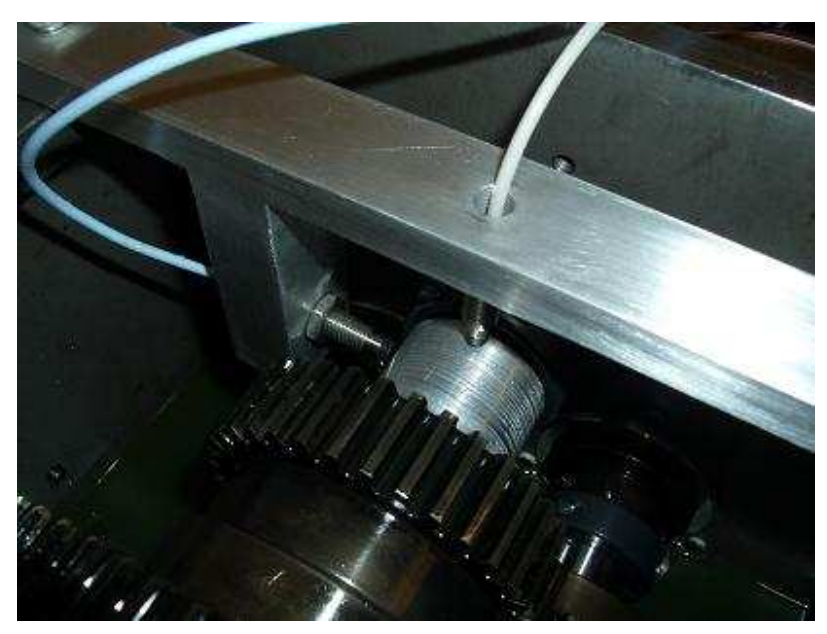

Figure 45: Gearbox Assembly with Bracket and Hub

\subsubsection{Accelerometers and ME'scopeVES}

Bearing frequencies and Gear frequencies could be collected by using accelerometers and a spectrum analyzer. Accelerometers will be attached onto the bearing housing on the gearbox, and the accelerometers output will be run through a ME'scopeVES interface box and be displayed on a computer. ME'scopeVES is a program that similar to a spectrum analyzer which allows users to collect and analyze vibration signals from a system. 


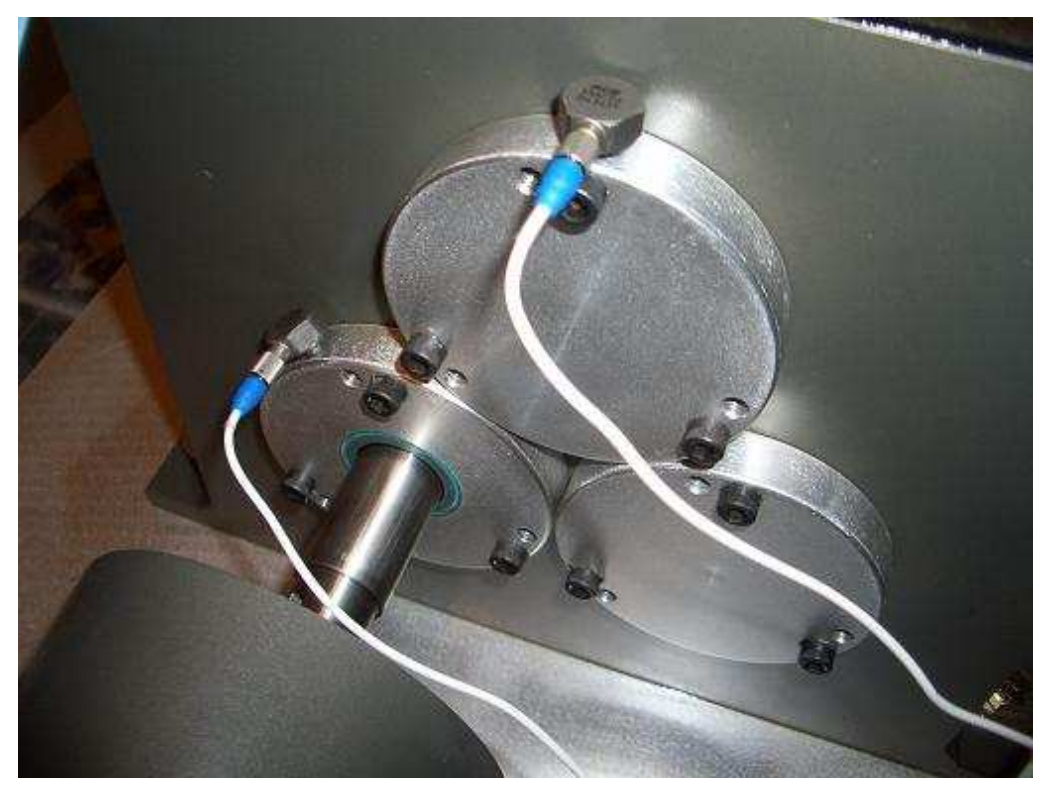

Figure 46: Accelerometers on housing

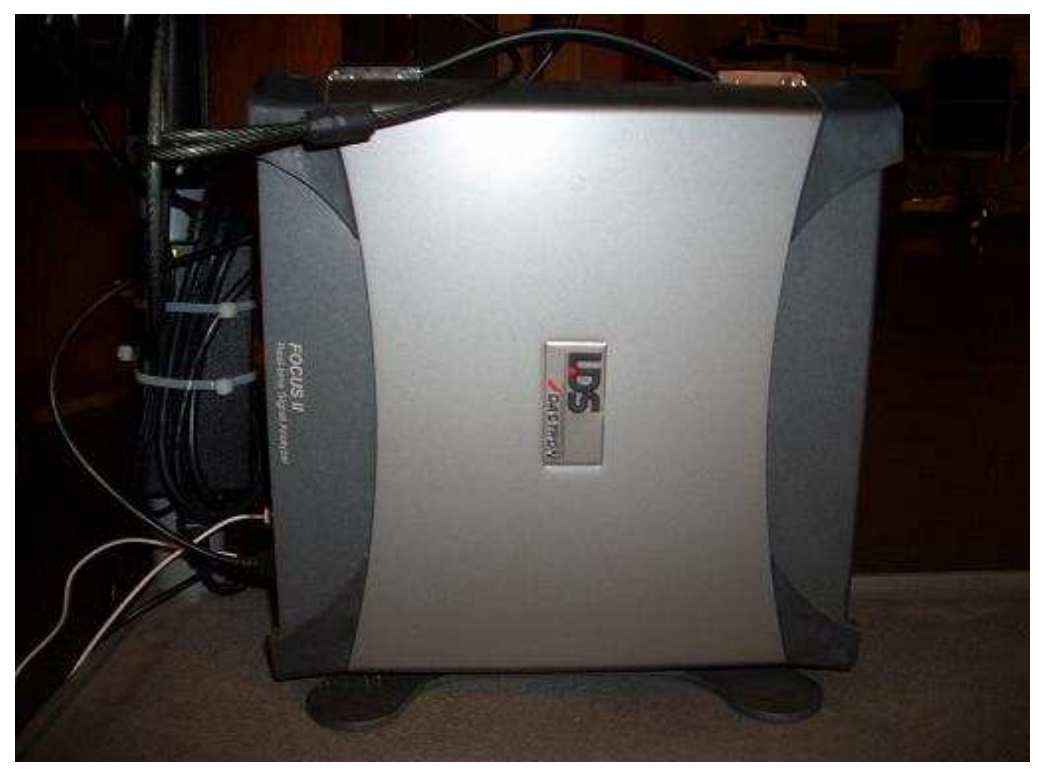

Figure 47: ME'scopeVES interface box 


\section{$5.5 \quad$ Results}

Results of the calculated frequencies vs. the experimental values are shown below.

Table 5: Calculated Values vs. Experimental Values at $17 \mathrm{~Hz}$ Input Frequencies

\begin{tabular}{|c|c|c|c|c|}
\hline \multicolumn{2}{|r|}{ Item } & Symbol & Calc. $(\mathrm{Hz})$ & Exp. $(\mathrm{Hz})$ \\
\hline \multirow{3}{*}{$\begin{array}{l}\text { Rotating } \\
\text { Frequencies }\end{array}$} & Shaft 1 (Input Shaft) Rotating Frequency & $\mathrm{f}_{1}$ & 17 & 17 \\
\hline & Shaft 2 (N2 and N3 Shaft) Rotating Frequency & $f_{2}$ & 6.8 & - \\
\hline & Shaft 3 (N4 Shaft) Rotating Frequency & $\mathrm{f}_{3}$ & 5.1 & - \\
\hline \multirow{2}{*}{$\begin{array}{c}\text { Meshing } \\
\text { Frequencies }\end{array}$} & First Stage Meshing Frequency & $\mathrm{f}_{\mathrm{m} 1}$ & 408 & 408.7 \\
\hline & Second Stage Meshing Frequency & $\mathrm{f}_{\mathrm{m} 2}$ & 244.8 & 245.4 \\
\hline \multirow{7}{*}{$\begin{array}{c}\text { Sub- } \\
\text { harmonic } \\
\text { Frequencies } \\
\text { of Meshing } \\
\text { Frequencies }\end{array}$} & 1/6 times of First Stage Meshing Frequency & $1 / 6 \times f_{\mathrm{m} 1}$ & 68 & 68.12 \\
\hline & 1/4 times of First Stage Meshing Frequency & $1 / 4 \times f_{\mathrm{m} 1}$ & 102 & 101.8 \\
\hline & 1/3 times of First Stage Meshing Frequency & $1 / 3 \times \mathrm{f}_{\mathrm{m} 1}$ & 136 & 136.2 \\
\hline & 1/2 times of First Stage Meshing Frequency & $1 / 2 \times f_{\mathrm{m} 1}$ & 204 & 204.3 \\
\hline & 2/3 times of First Stage Meshing Frequency & $2 / 3 \times f_{\mathrm{m} 1}$ & 272 & 272.5 \\
\hline & 1/2 times of Second Stage Meshing Frequency & $1 / 2 \times f_{\mathrm{m} 2}$ & 122.4 & 122.3 \\
\hline & 1/3 times of First Stage Meshing Frequency & $1 / 3 \times f_{\mathrm{m} 1}$ & 136 & 136.2 \\
\hline \multirow{6}{*}{$\begin{array}{c}\text { Super- } \\
\text { harmonic } \\
\text { Frequencies } \\
\text { of Meshing } \\
\text { Frequencies }\end{array}$} & 2 times of First Stage Meshing Frequency & $2 \times f_{\mathrm{m} 1}$ & 816 & 816.7 \\
\hline & 2 times of Second Stage Meshing Frequency & $2 \times f_{m 2}$ & 489.6 & 490 \\
\hline & 3 times of First Stage Meshing Frequency & $3 \times \mathrm{f}_{\mathrm{m} 1}$ & 1224 & 1225 \\
\hline & 3 times of Second Stage Meshing Frequency & $3 \times f_{\mathrm{m} 2}$ & 734.4 & 731.7 \\
\hline & 4 times of First Stage Meshing Frequency & $4 \times f_{\mathrm{m} 1}$ & 1632 & 1633 \\
\hline & 4 times of Second Stage Meshing Frequency & $4 \times f_{m 2}$ & 979.2 & 980 \\
\hline \multirow{19}{*}{$\begin{array}{l}\text { Bearing } \\
\text { Elements } \\
\text { Frequencies }\end{array}$} & \multirow{10}{*}{$\begin{array}{l}\text { Outer Race Ball Pass Frequency } \\
\text { and their Super-harmonic Frequencies }\end{array}$} & $\mathrm{f}_{\mathrm{ORBP}}$ & 116.22 & 119.4 \\
\hline & & $2 \times f_{\text {ORBP }}$ & 232.44 & 238 \\
\hline & & $3 \times \mathrm{f}_{\text {ORBP }}$ & 348.66 & 357.4 \\
\hline & & $4 \times \mathrm{f}_{\text {ORBP }}$ & 464.88 & 476.8 \\
\hline & & $5 \times f_{\text {ORBP }}$ & 581.1 & 595.5 \\
\hline & & $6 \times f_{\text {ORBP }}$ & 697.32 & 714.8 \\
\hline & & $7 \times \mathrm{f}_{\text {ORBP }}$ & 813.54 & 833.5 \\
\hline & & $8 \times \mathrm{f}_{\text {ORBP }}$ & 929.76 & 952.9 \\
\hline & & $9 \times f_{\text {ORBP }}$ & 1045.98 & 1055 \\
\hline & & $10 \times f_{\text {ORBP }}$ & 1162.2 & 1191 \\
\hline & \multirow{9}{*}{$\begin{array}{l}\text { Inner Race Ball Pass Frequency } \\
\text { and their Super-harmonic Frequencies }\end{array}$} & $\mathrm{f}_{\text {IRBP }}$ & 155.78 & 153.1 \\
\hline & & $2 \times f_{\text {IRBP }}$ & 311.56 & 306.2 \\
\hline & & $4 \times \mathrm{f}_{\text {IRBP }}$ & 623.12 & 612.3 \\
\hline & & $5 \times f_{\text {IRBP }}$ & 778.9 & 765.4 \\
\hline & & $6 \times f_{\text {IRBP }}$ & 934.68 & 919.2 \\
\hline & & $7 \times f_{\text {IRBP }}$ & 1090.46 & 1069 \\
\hline & & $8 \times \mathrm{f}_{\text {IRBP }}$ & 1246.24 & 1225 \\
\hline & & $9 \times f_{\text {IRBP }}$ & 1402.02 & 1378 \\
\hline & & $10 \times f_{\text {IRBP }}$ & 1557.8 & 1531 \\
\hline
\end{tabular}


The following figures are the experimental data generated from gearbox dynamics simulator and collected by ME'scopeVES. Figure 48 shows the power spectrum of the system in a frequency range of 0 to $2000 \mathrm{~Hz}$. Since it is hard to identify frequencies in this range, the plot is separated into 3 sub-plots in order to indentify the important frequencies.

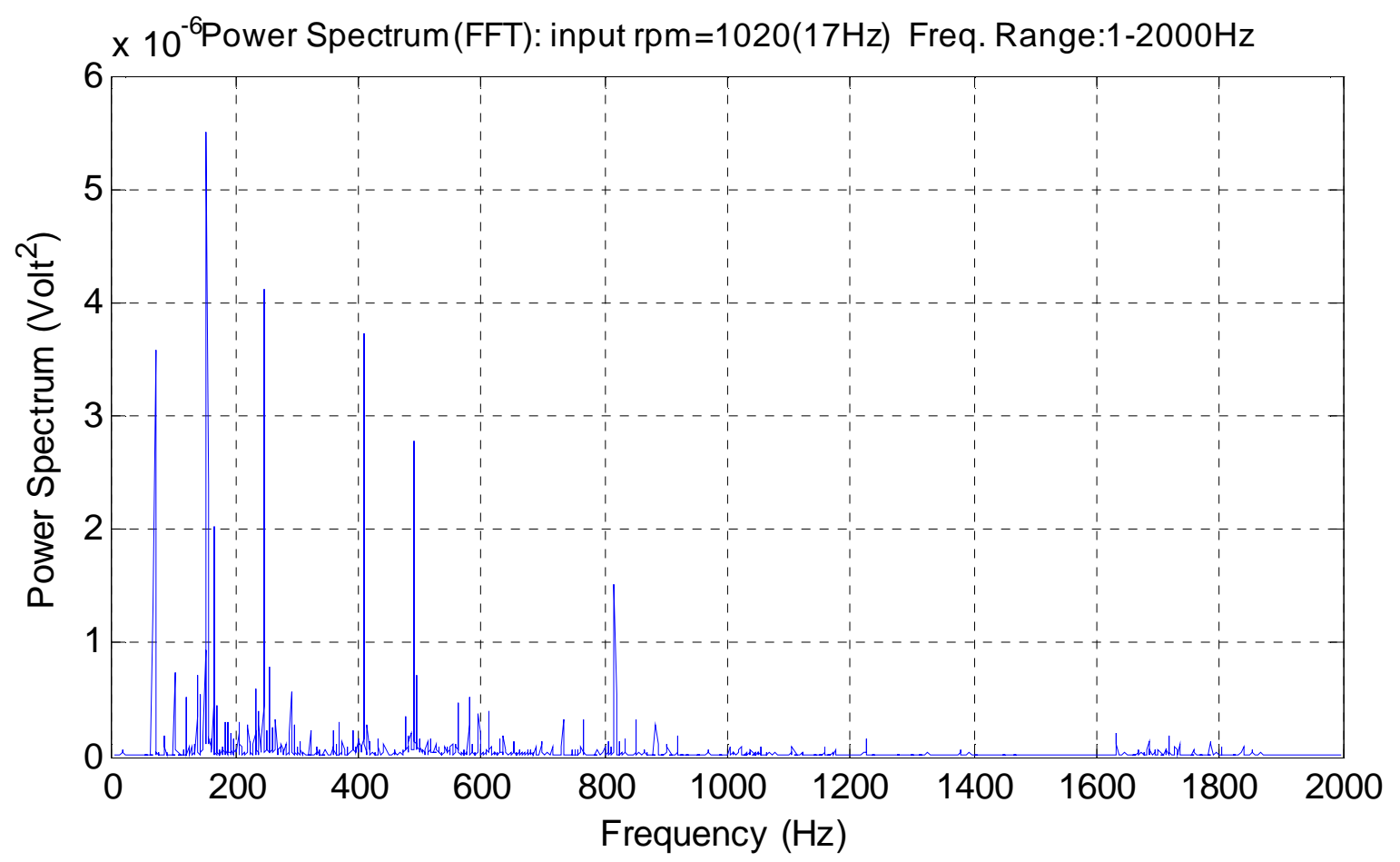

Figure 48: Power Spectrum Plot with frequency range between 0 to $2000 \mathrm{~Hz}$ 


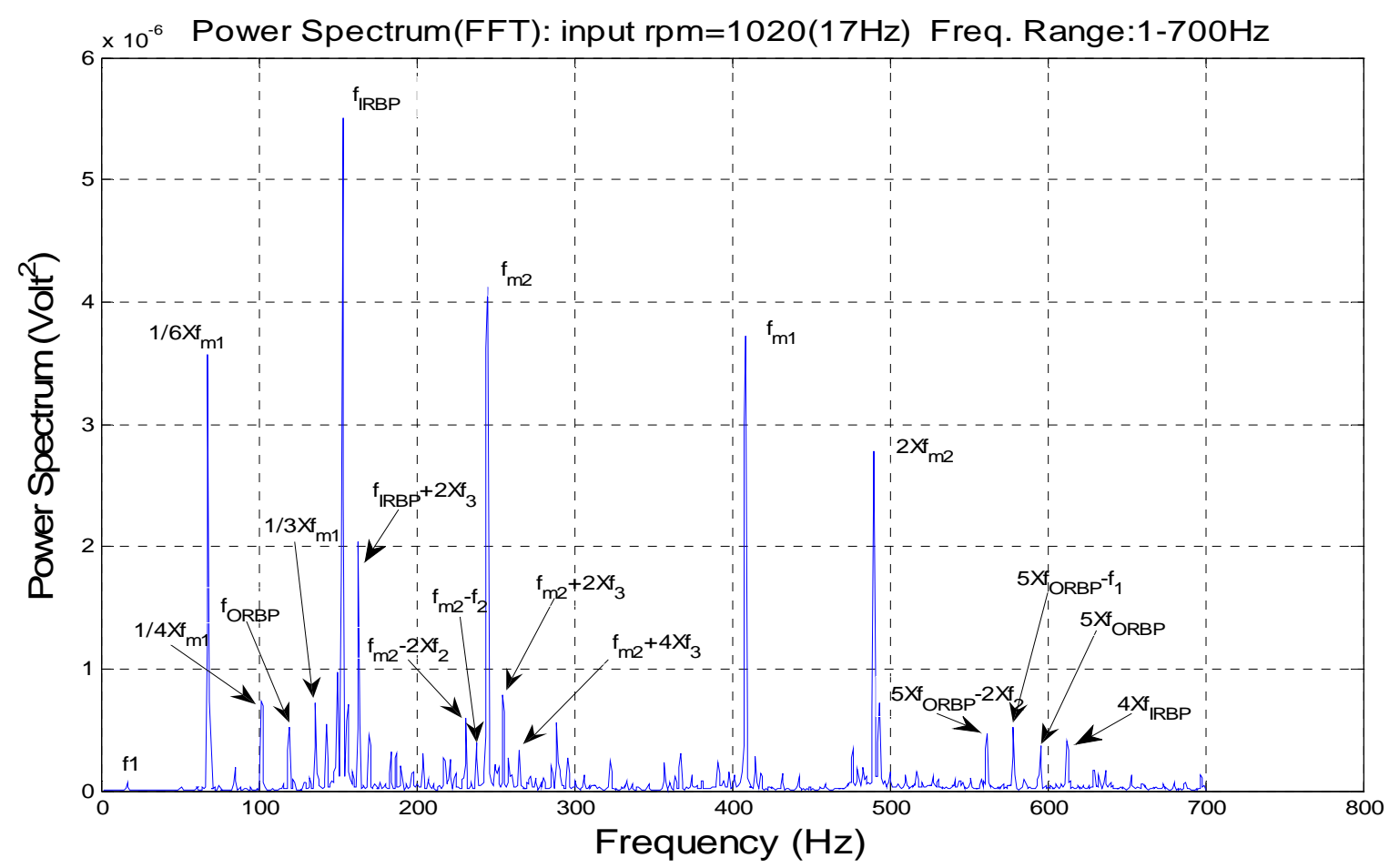

Figure 49: Power Spectrum Plot with frequency range between 1 to $700 \mathrm{~Hz}$

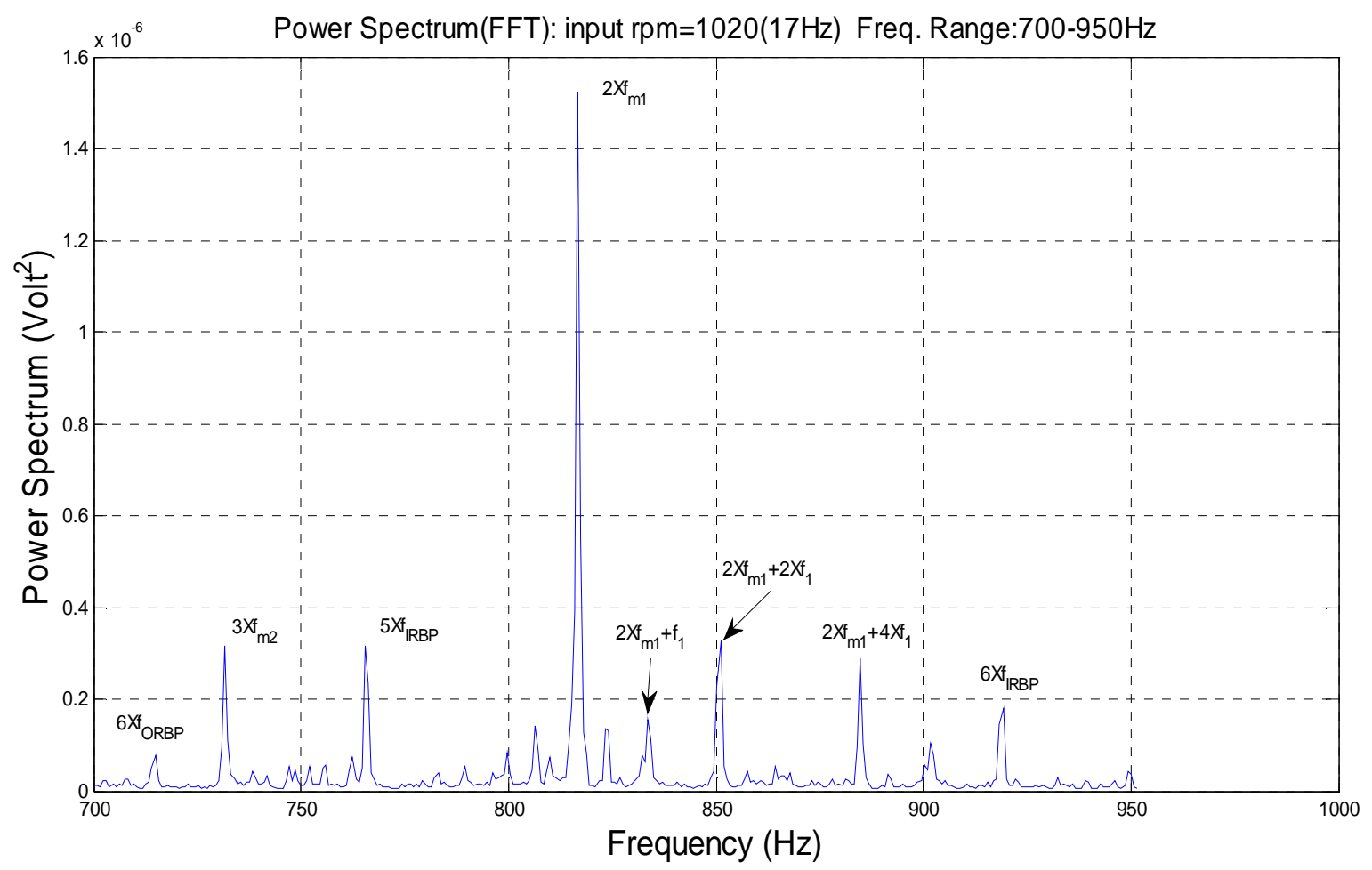

Figure 50: Power Spectrum Plot with frequency range between 700 to $950 \mathrm{~Hz}$ 


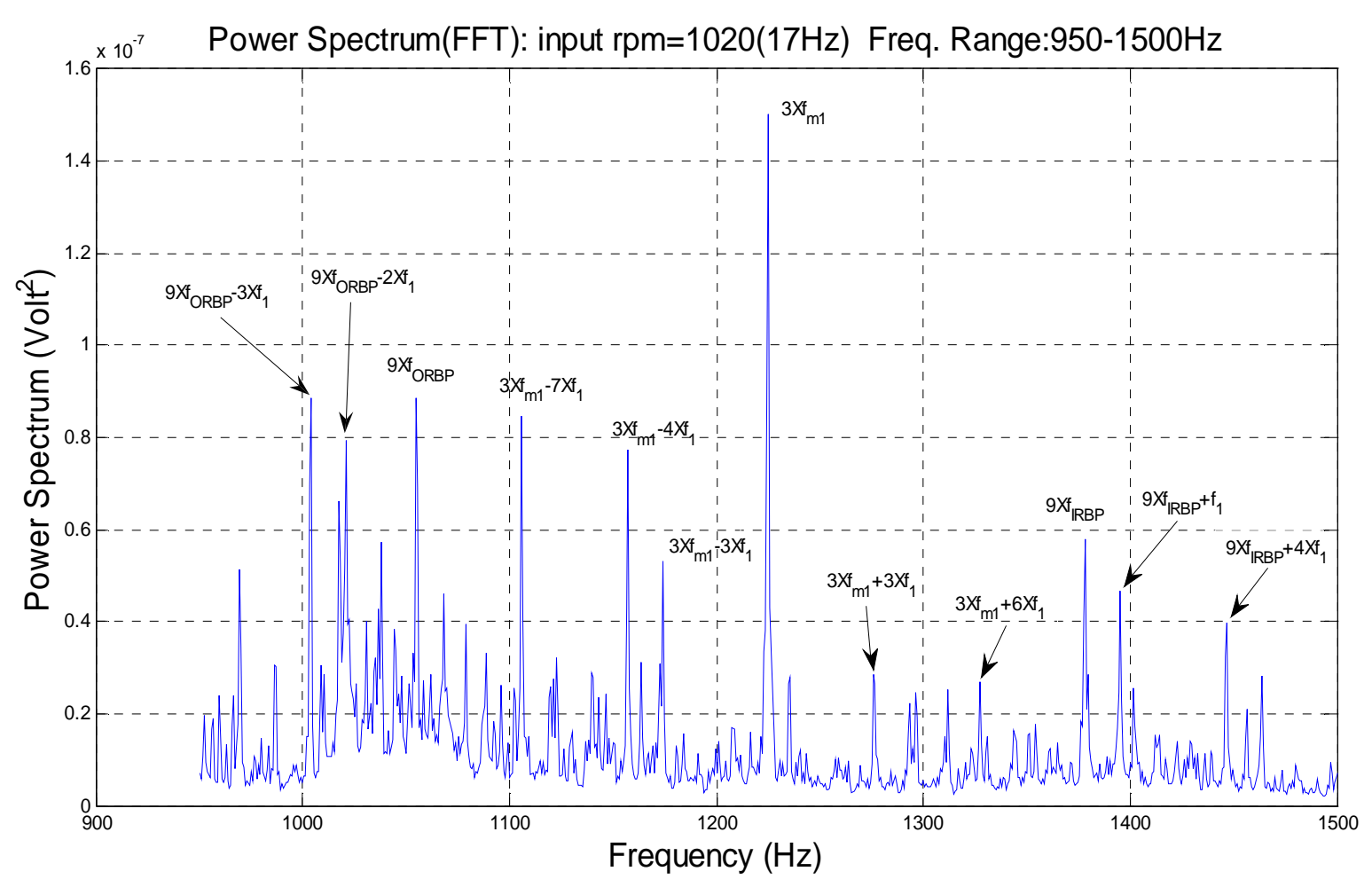

Figure 51: Power Spectrum Plot with frequency range between 950 to $1500 \mathrm{~Hz}$

The results show that the experimental values match the calculated values closely when the gearbox dynamics simulator is operating at $17 \mathrm{~Hz}$. To confirm the system is operating without fault, a different operating speed, $25 \mathrm{~Hz}$, is chosen. The following table and figures are the results of the system operating at $25 \mathrm{~Hz}$ in a frequency range of 0 to $2000 \mathrm{~Hz}$. Again, the power spectrum plot is separated into 3 sub-plots in order to capture the important frequencies. 
Table 6: Calculated Values vs. Experimental Values at $25 \mathrm{~Hz}$ Input Frequencies

\begin{tabular}{|l|c|c|c|}
\hline \multicolumn{1}{|c|}{ Item } & Symbol & Calc. Value $(\mathrm{Hz})$ & Exp. Value $(\mathrm{Hz})$ \\
\hline Input Shaft Rotating Frequency & $\mathrm{f}_{1}$ & 25 & 25 \\
\hline First Stage Meshing Frequency & $\mathrm{f}_{\mathrm{m} 1}$ & 600 & 600.6 \\
\hline Second Stage Meshing Frequency & $\mathrm{f}_{\mathrm{m} 2}$ & 360 & 360.4 \\
\hline $1 / 4$ times of First Stage Meshing Frequency & $1 / 4 \times \mathrm{f}_{\mathrm{m} 1}$ & 150 & 150.1 \\
\hline $1 / 2$ times of First Stage Meshing Frequency & $1 / 2 \times \mathrm{f}_{\mathrm{m} 1}$ & 300 & 300.3 \\
\hline $1 / 2$ times of Second Stage Meshing Frequency & $1 / 2 \times \mathrm{f}_{\mathrm{m} 2}$ & 180 & 185.3 \\
\hline $1 / 3$ times of First Stage Meshing Frequency & $1 / 3 \times \mathrm{f}_{\mathrm{m} 1}$ & 200 & 200 \\
\hline $1 / 3$ times of Second Stage Meshing Frequency & $1 / 3 \times \mathrm{f}_{\mathrm{m} 2}$ & 120 & 125.2 \\
\hline 2 times of First Stage Meshing Frequency & $2 \times \mathrm{f}_{\mathrm{m} 1}$ & 1200 & 1200 \\
\hline 2 times of Second Stage Meshing Frequency & $2 \times \mathrm{f}_{\mathrm{m} 2}$ & 720 & 725.1 \\
\hline 3 times of First Stage Meshing Frequency & $3 \times \mathrm{f}_{\mathrm{m} 1}$ & 1800 & 1801 \\
\hline 3 times of Second Stage Meshing Frequency & $3 \times \mathrm{f}_{\mathrm{m} 2}$ & 1080 & 1080 \\
\hline 4 times of Second Stage Meshing Frequency & $4 \times \mathrm{f}_{\mathrm{m} 2}$ & 1440 & 1441 \\
\hline
\end{tabular}

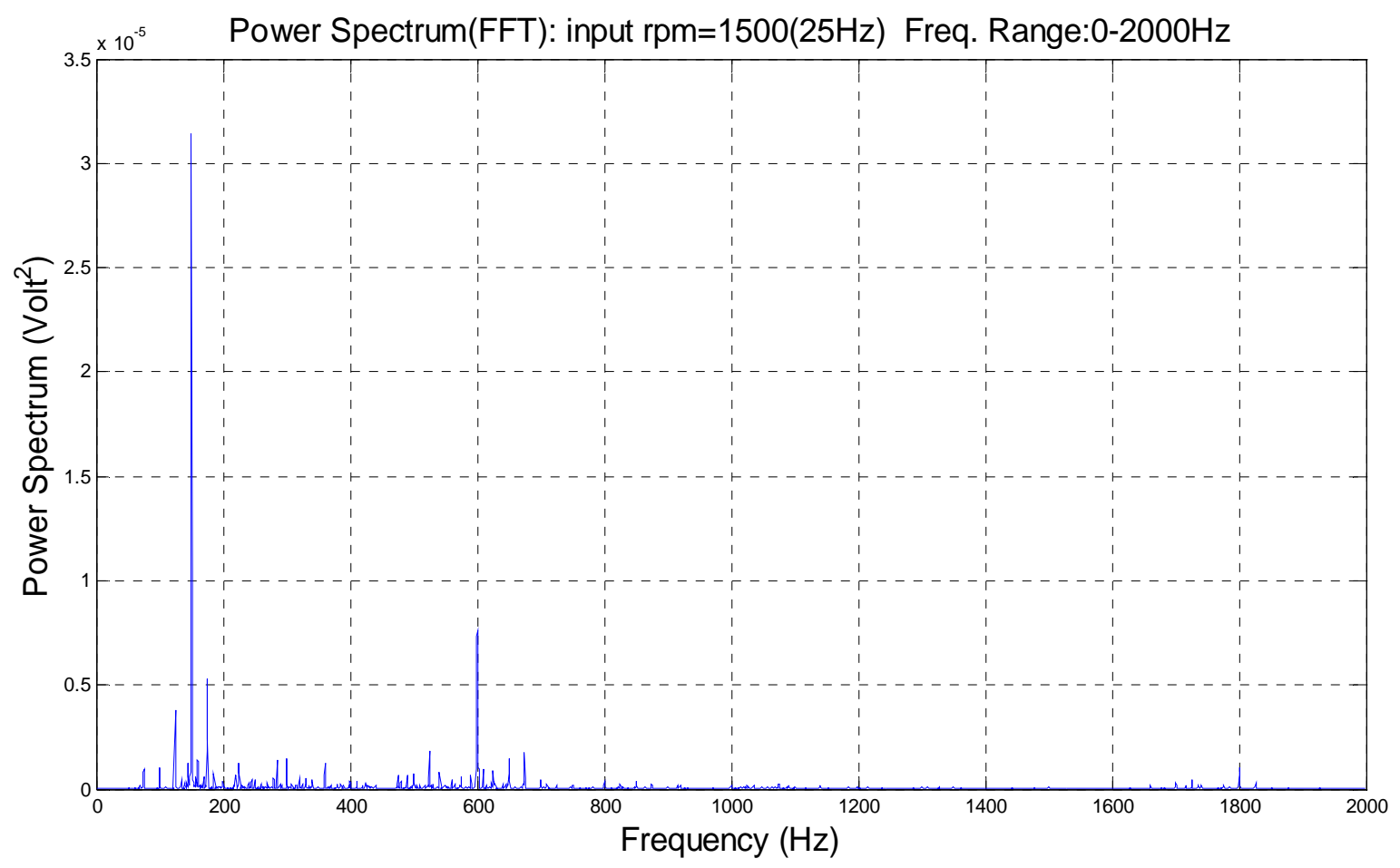

Figure 52: Power Spectrum Plot with frequency range between 0 to $2000 \mathrm{~Hz}$ 


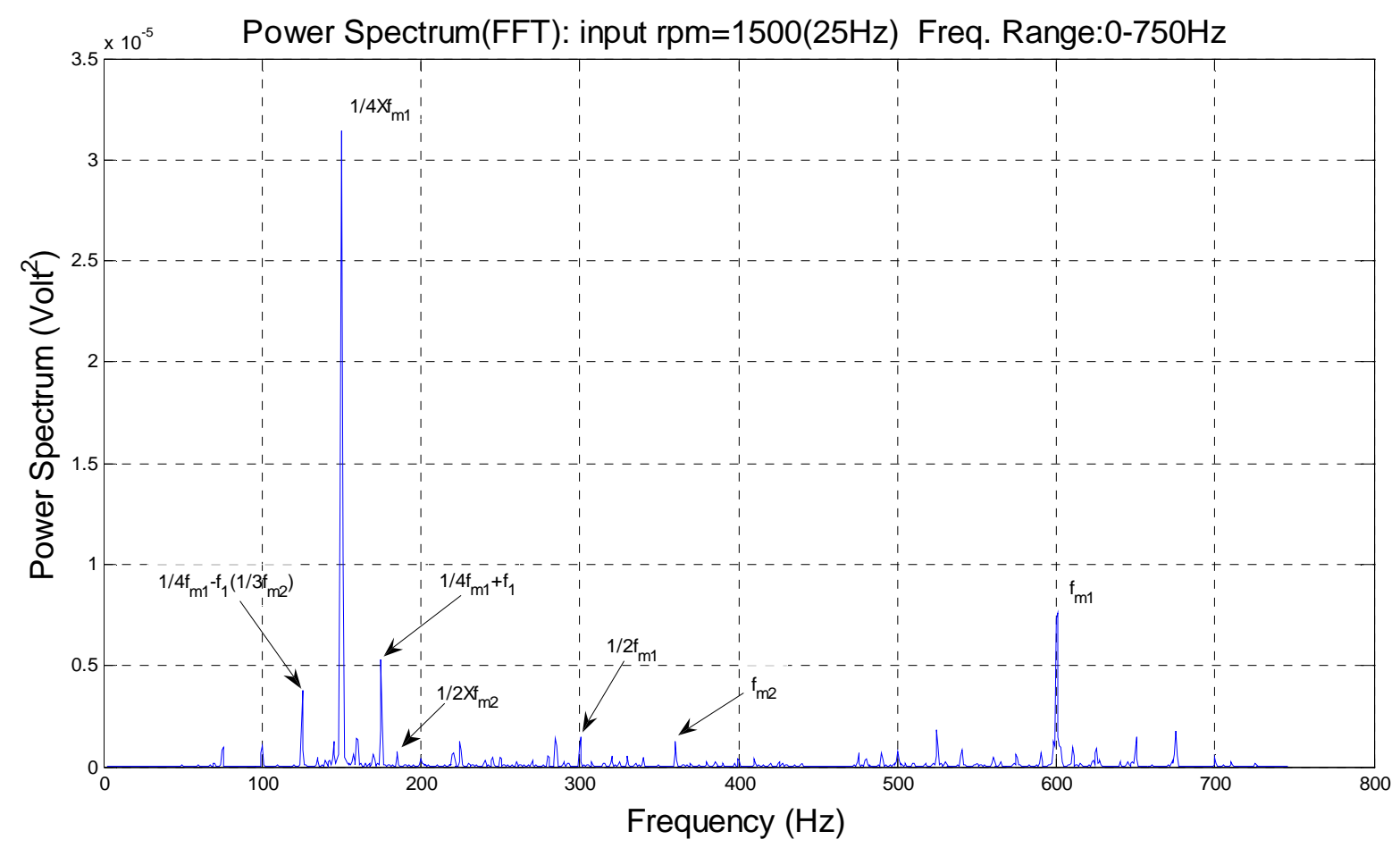

Figure 53: Power Spectrum Plot with frequency range between 0 to $750 \mathrm{~Hz}$

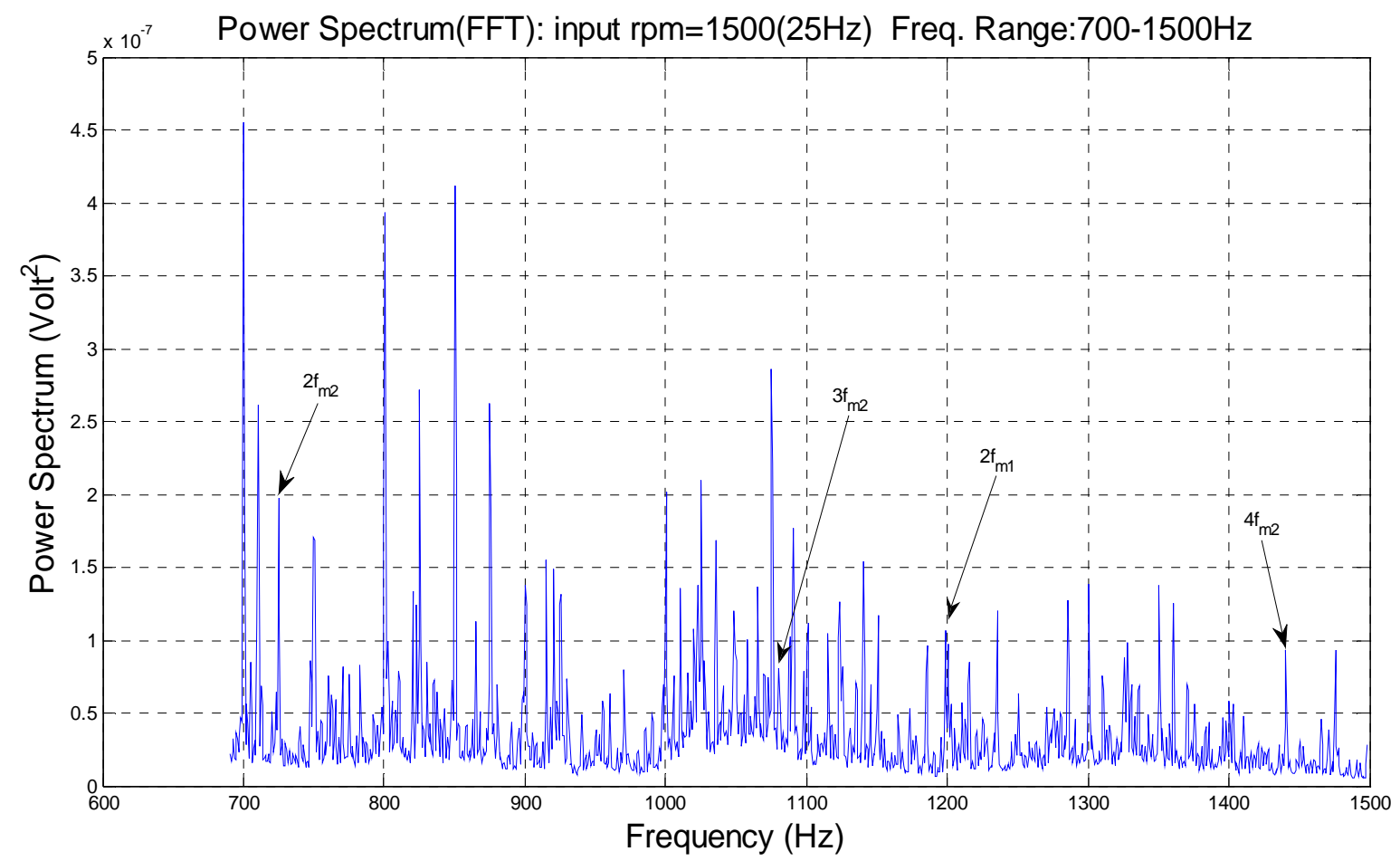

Figure 54: Power Spectrum Plot with frequency range between 700 to $1500 \mathrm{~Hz}$ 


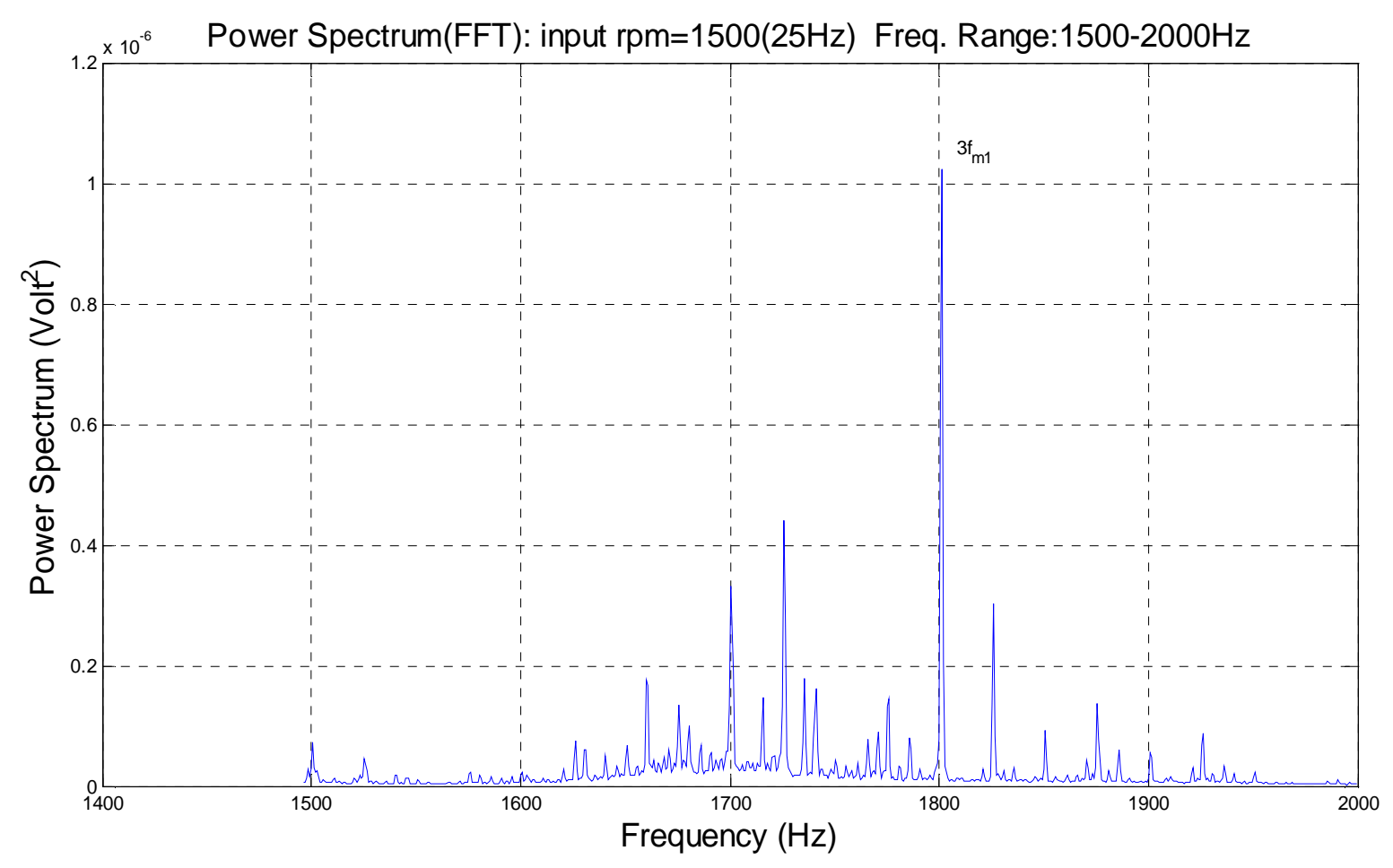

Figure 55: Power Spectrum Plot with frequency range between 1500 to $2000 \mathrm{~Hz}$

Once again, the results show that the experimental values match the calculated values closely even when the gearbox dynamics simulator is operating at different speed. 


\section{Results and Conclusions}

The contact between the pinion and gear is highly non-linear because the surfaces of the tooth could come into and out of contact in a sudden manner. Due to the nonlinear effect, the meshing stiffness alternates between the single tooth contact zone and the double tooth contact zone. Finite element gear models with quasi-static method have been made to simulate the meshing stiffness through the meshing cycle. Based on the results, the FE gear models have generated reasonable solutions, and by reasonable, it means results are similar to those from previous works. As mentioned before, FEA solution should be compared with closed-form calculation or theoretical models in order to evaluate its reliability. A theoretical model [20] has been developed and compared with the FEA result; the comparison is shown below.

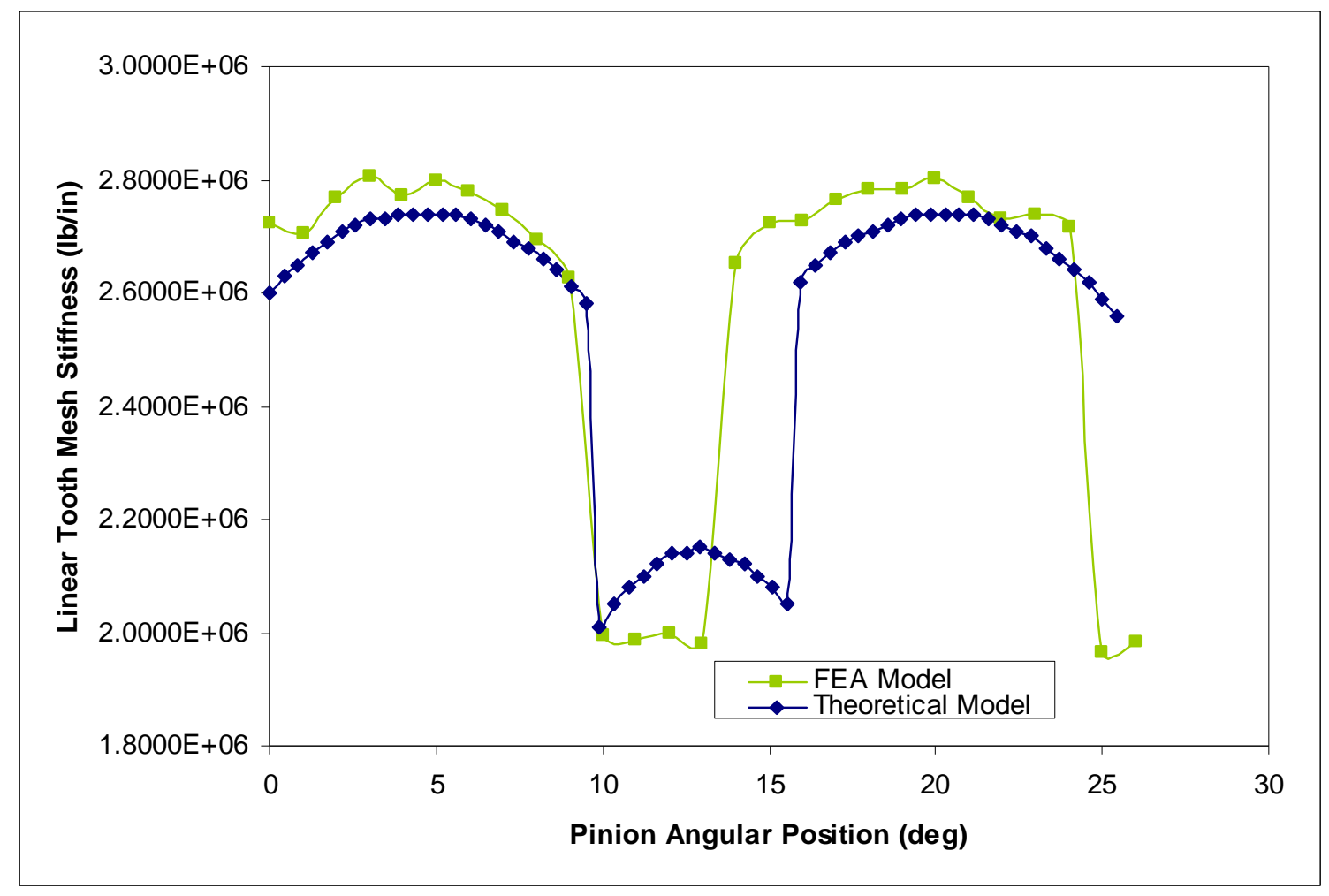

Figure 56: Comparison between FEA and Theoretical Models 
From Figure 56, it shows that the general shape of the curve from FEA matches the theoretical model, and the stiffness values are in range. It is clear that fine tuning is required for the FEA model especially at the single tooth contact zone. In addition, the closed-form solution discussed in section 3.6 has been calculated, and the meshing stiffness in a single tooth contact zone results in a value of $2.206 \mathrm{e} 6 \mathrm{lb} / \mathrm{in}$. Compared to the FEA value $2.000 \mathrm{e} 6 \mathrm{lb} / \mathrm{in}$, there is a $9.3 \%$ difference. This difference is expected since the closed-form solution employs only a short rectangular beam instead of an actual gear tooth and ignores Hertz contact stresses and gear body compliance. These comparisons only show the FEA simulations in this work are within a reliable range; major refinement is essential if further works and comparisons are required.

Furthermore, one of the ideal forms of comparison would be utilizing experimental testing. However, experimental activities could be expensive and lengthy. In this particular case, obtaining meshing stiffness through experiments require special set up and equipment. A torque sensor will be needed for measuring the input torque. Strain gauges will be needed to measure the torsional displacement of the gear. The strain gauges have to be wireless. As one might have guessed, if the normal wired strain gauges are installed on the gears in a gearbox, they would have been destroyed as soon as the machine starts to rotate. Because of that, a different set up (not confined by the gearbox casing) is needed. The level of intensity of this experiment is beyond the scope of this work. Further work such as FEA model refinement and experiment testing is suggested for the future. 


\section{References}

[1] Wyluda, P. and Wolf, D. Examination of finite element analysis and experimental results of quasi-statically loaded acetal copolymer gears.

[2] Barone, S., Borgianni, L. and Forte, P. (2003). Evaluation of the effect of misalignment and profile modification in face gear drive by a finite element meshing simulation. 2003 Design Engineering Technical Conferences and Computers and Information in Engineering Conference. ASME.

[3] Howard, I., Jia, S. and Wang, J. (2001). The dynamic modeling of a spur gear in mesh including friction and a crack. Mechanical Systems and Signal Processing (2001) 15(5), 831-853.

[4] Wang, J. and Howard, I. (2005). Finite Element Analysis of High Contact Ratio Spur Gears in Mesh. Journal of Tribology. ASME. Vol. 127, 469-483.

[5] Wang, J. and Howard, I. (2006). Error Analysis on Finite Element Modeling of Involute Spur Gears. Journal of Mechanical Design. ASME. Vol. 128, 90-97.

[6] Carmignani, C., Forte, P. and Melani G. (2007). Study on diagnostic indictors of gear tooth crack length and location in a virtual gear transmission. ISCORMA-4, Calgary, Alberta, Canada 27-30 August 2007.

[7] Drago, R.J. (1988). Fundamentals of Gear Design. Division of Reed Publishing Inc. Butterworth Publishers. USA.

[8] Shigley, J.E., Mischke, C.R. and Budynas, R.G. (2004). Mechanical Engineering Design. Seventh Edition. McGraw-Hill Companies Inc. New York.

[9] Machinery's Handbook. 28 ${ }^{\text {th }}$ Edition. Industrial Press Inc. New York.

[10] Master's Thesis. (2006 - 2007). Software Development on Finite Element Analysis for Bending Stresses of Transmission Gears. Ji Ling University, China.

[11] Adjustment of the MatLab Code from [10]. See Appendix.

[12] Getting Started with Abaqus: Interactive Edition. Version 6.7, Contact. Chapter 12.

[13] Abaqus Theory Manual. Version 6.7, Interface Modeling. Chapter 5.

[14] Master's Thesis. Online source. Curtin University of Technology, Australia. http://adt.curtin.edu.au/theses/available/adt-WCU20040323.133144/unrestricted. 
[15] Abaqus Analysis User's Manual. Version 6.7, Elements: Introduction and Continuum Elements. Chapter 21 - 22.

[16] Abaqus Theory Manual. Version 6.7, Element library: overview. Chapter 3, Section 3.1.1.

[17] Abaqus/CAE User's Manual. Version 6.7, Mesh module basics. Chapter 17, Section 17.3.

[18] Arthur, P. and Chong, K.P. (1987). Elasticity in Engineering Mechanics, New York: Elsevier.

[19] Richards, R.R. (2001). Principles of solid mechanics, Boca Raton, Fla.: CRC Press, c2001.

[20] Meagher, J., Wu, X., Kong, D., Lee, C.H., "A Comparison of Gear Mesh Stiffness Modeling Strategies", IMAC XXVIII a Conference on Structural Dynamics, Society for Experimental Mechanics, Jacksonville, Florida USA, February $1-4,2010$.

[21] Ural, A., Wawrzynek, P.A. and Ingraffea, A.R. (2003). Simulating Fatigue Crack Growth in Spiral Bevel Pinion. NASA/CR-2003-212529. National Aeronautics and Space Administration. Glenn Research Center. 


\section{Appendix: MatLab Code for gear tooth profile}

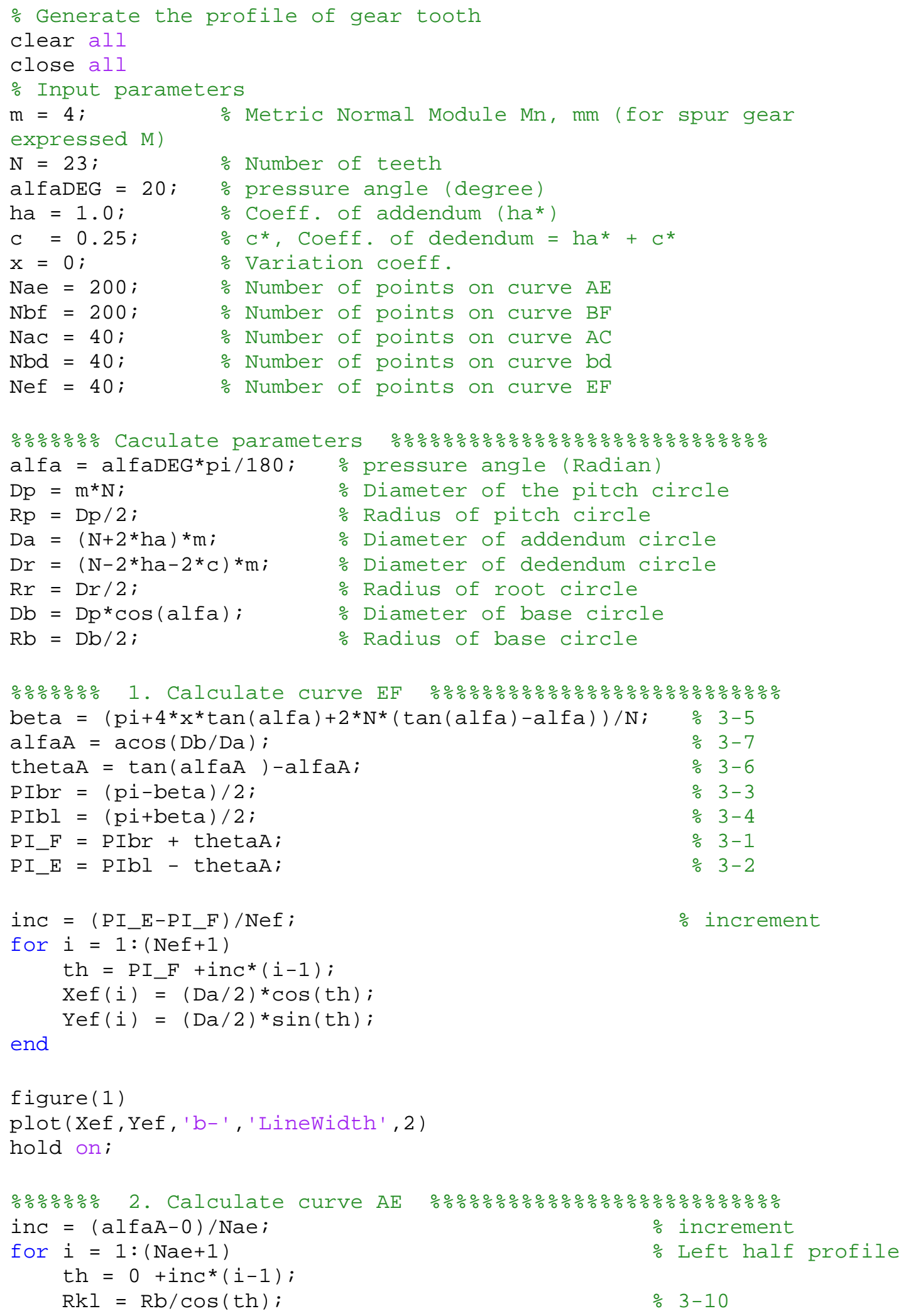




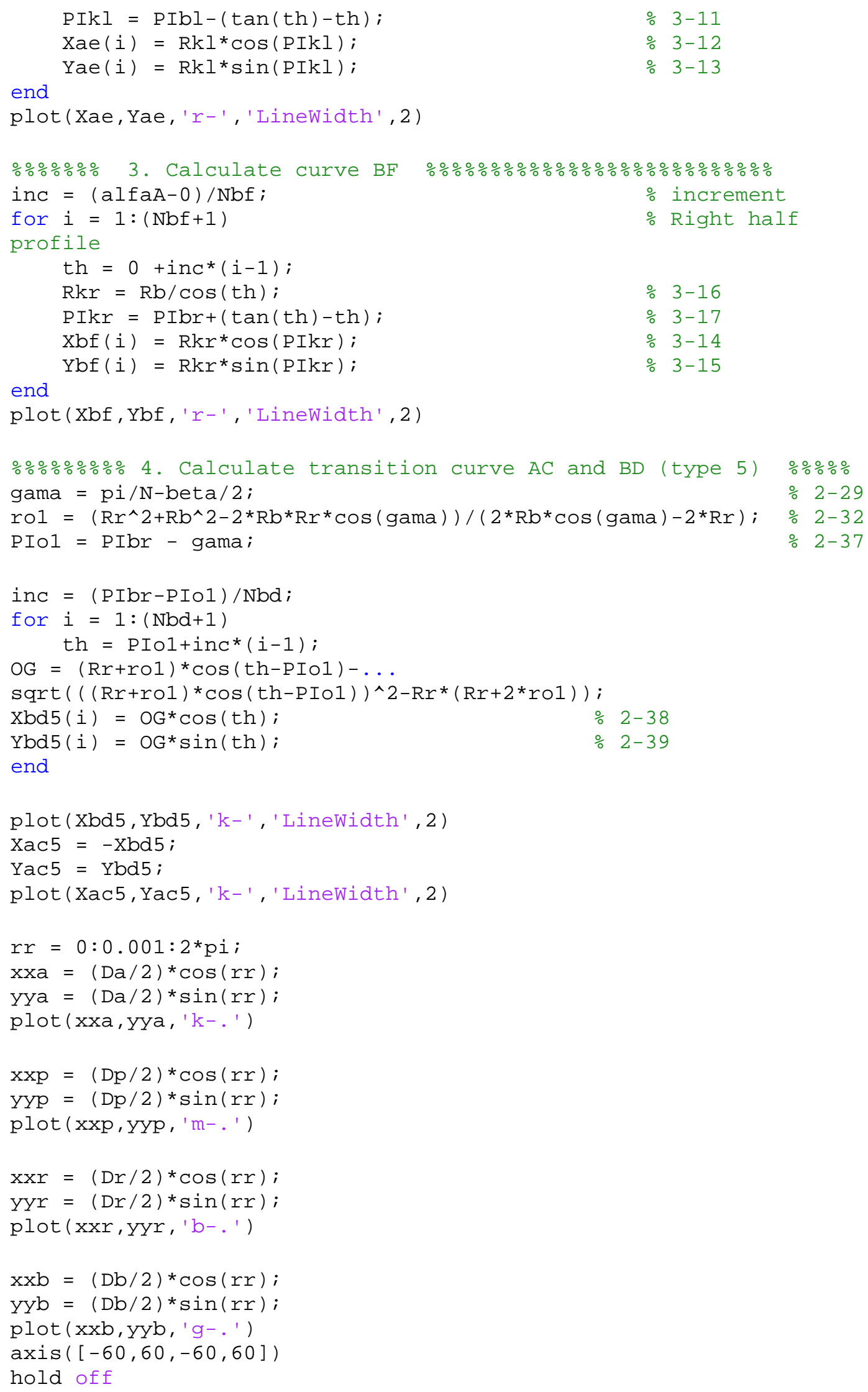

